\title{
ANISOTROPIC PHASE SEPARATION THROUGH THE METAL-INSULATOR TRANSITION IN AMORPHOUS Mo-Ge AND Fe-Ge ALlOYS
}

\author{
Michael J. Regan \\ Stanford Linear Accelerator Center \\ Stanford Synchrotron Radiation Laboratory \\ Stanford University, Stanford, California 94309
}

December 1993

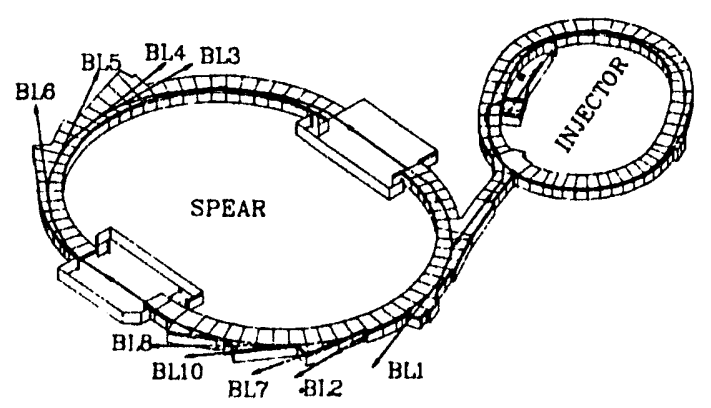

Prepared for the Department of Energy under contract number DE-AC03-76SF00515

Printed in the United States of America. Available from the National Technical Information Service, U.S. Department of Commerce, 5285 Port Royal Road, Springfield, Virginia 22161 


\section{Abstract}

Since an amorphous solid is often defined as that which lacks long-range order, the atomic structure is typically characterized in terms of the high-degree of short-range order. Most descriptions of vapor-deposited amorphous alloys focus on characterizing this order, while assuming that the material is chemically homogeneous beyond a few near neighbors. Phase separation, which leads to a structure with regions of different short-range order, is, however, endemic in the quasi-equilibrium states of such materials. By coupling traditional small-angle $\mathrm{x}$-ray scattering which probes spatial variations of the electron density with anomalous dispersion which creates a species-specific contrast, we can discern cracks and voids from chemical inhomogeneity.

In particular, we find that the chemical inhomogeneities which have been previously reported in amorphous $\mathrm{Fe}_{x} \mathrm{Ge}_{1 \cdot x}$ and $\mathrm{Mo}_{x} \mathrm{Ge}_{1-x}$ are quite anisotropic, depending significantly on the direction of film growth. With the addition of small amounts of metal atoms $(x<0.2)$, no films appear isotropic nor homogeneous through the metal/insulator transition. The results indicate that fluctuations in the growth direction play a pivotal role in preventing simple growth models of a columnar structure or one that evolves systematically as it grows. The anisotropy is interpreted in real-space with the cylindrical correlation function, which has been calculated using a spherical harmonics approach. The anisotropic phase separation can be modeled as a collection of elongated particles, oriented with the long axis in the direction of growth, which show strong "inplane" correlations but little, if any, in the cirection of film growth.

The anomalous scattering measurements identify the metal atoms ( $\mathrm{Fe}$ or $\mathrm{Mo}$ ) as the source of the anisotropy, with the Ge atoms distributed homogeneously. We have developed a method for using these measurements to determine the compositions of the 
phase-separating species. Our results indicate phase separation into an amorphous $\mathrm{Ge}$ and an intermetallic phase of stoichiometry close to $\mathrm{FeGe}_{2}$ or $\mathrm{MoGe}_{3}$. Finally, by manipulating the deposited power flux and rates of growth, $\mathrm{Fe}_{\mathrm{x}} \mathrm{Ge}_{1 \cdot \mathrm{x}}$ films which have the same Fe composition $x$ can be grown to different states of phase separation. These results may help explain the difficulty workers have had in isolating the metal/insulator transition for these and other vapor-deposited amorphous alloys. 


\section{Acknowledgments}

I would like to thank several people for their support and friendship over the past five years that has made my experience at Stanford University memorable. Special thanks to my advisor Artie Bienenstock who brought me into his research group and treated me as a true collaborator and working scientist. His motivation, critical analysis, and insight to both experimental and theoretical issues provided a unique learning experience that I hope to emulate as a scientist.

The topic chosen for this work, anomalous small-angle $x$-ray scattering (ASAXS) from amorphous films, relied heavily on early work of Marybeth Rice, to whom I am particularly grateful. The opportunity to work with Marcela Fernandez van Raap in a study of amorphous Fe-Si was also a key element to my education at Stanford and SSRL. Besides enjoying her friendship, I learned immensely from Marcela's experiences and viewpoints and from teaching her about sputtering and ASAXS. The work with Ritva Serimaa (ASAXS of Pt complexes) and Pierre LeCante (grazing-incidence ASAXS) and the consequent discussions of technical problems and data interpretation are also memorable.

In addition, I would like to thank Hiro Tsuruto, David Eliezer, Jeff Pape, Tom Russell, Hal Tompkins, Sean Brennan, and the SSRL X-ray Group for their assistance and helpful suggestions with the ASAXS camera, beamline optics, and beamline electronics.

Finally and most importantly, I would like to thank my wife Martha, my parents Mike and Barbara, and my brother and sister Jim and MaryNell for their unconditional support and commitment to my education and personal growth. 


\section{Contents}

Abstract $\quad$ ii

Acknowledgements iv

1 Introduction and statement of problem 1

1.1 Metal-Insulator Transition 2

1.2 Structural Models and Previous Work 3

$\begin{array}{lll}1.3 & \text { New Interests } & 6\end{array}$

$\begin{array}{ll}\text { References for Chapter } 1 & 10\end{array}$

2 Sample Preparation and Characterization 12

2.1 Sample Preparation $\quad 12$

2.2 Initial Characterization 14

2.3 X-Ray Absorption Measurements 16

References for Chapter 2

3 ASAXS Experiment and Data Treatment 22

3.1 Structure Factor and Pair Correlation Functions 22

3.2 Differential Anomalous Scattering 28

3.2.a Weighted Average PSFs 29 
3.2.b Keating and Munro Approaches $\quad 30$

$\begin{array}{lll}3.3 & X-R a y ~ O p t i c s & 32\end{array}$

3.4 SSRL Small-Angle Scattering Camera 36

3.5 Data Normalization and the Absolute Scale 40

3.6 Data Collection and the Measured Structure Factor 47

References for Chapter $3 \quad 62$

4 Amorphous $\mathrm{Fe}_{x} \mathrm{Ge}_{1 \cdot x}$ Results 64

4.1 Anisotropy and DAS Results 64

4.2 Mean Square Fluctuation in Effective Electron Density 71

$\begin{array}{lll}\text { 4.2.a Calculation and Values } & 71\end{array}$

$\begin{array}{lll}\text { 4.2.b Sharp Transition } & 75\end{array}$

$\begin{array}{lll}\text { 4.2.c Broad Transition } & 79\end{array}$

4.3 Cylindrical Correlation Functions (CCFs) 80

4.3.a Theory of the CCF 82

4.3.b Application of CCF to Amorphous Films 84

$\begin{array}{ll}\text { References for Chapter } 4 & 89\end{array}$

5 Amorphous $\mathrm{Mo}_{\mathbf{x}} \mathrm{Ge}_{1 \cdot x}$ Results $\quad 90$

$\begin{array}{lll}5.1 \text { Anisotropy } & 90\end{array}$

5.2 DAS Results 96

5.3 Mean-Square Fluctuation in Density 98

6 Models of Phase Separation $\quad 100$

6.1 Simple Two-Dimensional Growth Picture 101

$\begin{array}{lll}\text { 6.1.a Static Structures } & 101\end{array}$ 
6.1.b Dynamic Structure: Surface Evolution 103

$\begin{array}{ll}6.2 & \text { Single Particle Models } \\ \end{array}$

6.2.a Homogeneous Single Particles 109

6.2.b Inhomogeneous Single Particles 111

6.3 Close-Packed Homogeneous Particles 118

6.3.a Relations Between Interparticle Correlation Functions 119

6.3.b Hard-Core Percus-Yevick Approximation 121

$\begin{array}{ll}\text { 6.3.c Results of Model } & 127\end{array}$

6.4 Experimentally Obtained Interparticle Interference Function 134

$\begin{array}{ll}\text { References for Chapter } 6 & 144\end{array}$

7 Summary and Conclusions $\quad 145$

7.1 Identification of Phase-Separating Species $\quad 145$

7.2 Source of Anisotropic Scattering $\quad 147$

7.3 Metal-Insulator Transition 150

$\begin{array}{lll}7.4 & \text { New Questions } & 150\end{array}$

$\begin{array}{ll}\text { References for Chapter } 7 & 152\end{array}$ 


\section{List of Tables}

2.1 Summary of deposition conditions for samples studied 20

3.1 Darwin widths and energy resolution for Si mono crystals 34

3.2 Results of large $k$ fits to select samples 53

4.1 Correlation parameters from CCF for 6.3 at.\% Fe sample 87

5.1 Correlation parameters from CCF for 6.5 at.\% Mo sample 95

5.2 Computed endpoint compositions for Mo-Ge alloys 99 


\section{List of Figures}

2.1 Raw absorption data and extracted ASFs for 23.6 at.\% Mo at Ge K-edge $\quad 18$

3.1 Spherical coordinate system 25

3.2 Scattering geometry and SAXS camera 33

3.3 Energy resolution and the influence of focusing elements 35

3.4 Comparison of $\mathrm{Xe} / \mathrm{CO}_{2}$ and $\mathrm{Ar} / \mathrm{CO}_{2}$ detector gas efficiencies 39

3.5 Absorption correction illustration $\quad 42$

3.6 Subtraction of scattering from window materials 49

3.7 Plots to extract interfacial width 52

$3.8 \log -\log$ plots to determine large $k$ power law 54

3.9 Comparison between interfacial width and power-laws 56

3.10 Influence of incoherent scattering on the power-law $\alpha \quad 57$

3.11 Difficulty in determining $\alpha$ with short $k$ range $\quad 59$

3.12 Background subtracted data for 13.6 at.\% Fe 60

4.1 Oblique transmission results for $\mathrm{Fe}_{\mathrm{x}} \mathrm{Ge}_{1-\mathrm{x}} \quad 65$

4.2 SAXS from $\mathrm{Fe}_{\mathrm{x}} \mathrm{Ge}_{1 \cdot \mathrm{x}}$ for different target power settings 67

4.3 Weighted-average PSFs for 13.6 at.\% Fe sample 69

4.4 Munro PSFs for the 13.6 at.\% Fe sample 70

4.5 Mean-square fluctuation of effective electron density for $\mathrm{Fe}_{\mathrm{x}} \mathrm{Ge}_{1 \cdot \mathrm{x}}$ samples 74

4.6 Endpoint compositions predicted from two- and three-phase models 77 
4.7 Different interface models for sharp and broad particle boundaries

4.8 Interpretation of correlation function for SAXS studies

4.9 CCF Legendre coefficients $S_{n}(k)$ for 6.3 at.\% Fe sample

4.10 CCF for 6.3 at.\% Fe sample

5.1 Normal transmission SAXS results for several $\mathrm{Mo}_{\mathrm{x}} \mathrm{Ge}_{1-\mathrm{x}}$ samples

5.2 Oblique transmission results for 6.5 at.\% Mo sample

5.3 CCF for 6.5 at.\% Mo sample

5.4 Weighted-average PSFs for 6.5 at.\% Mo sample

6.1 Theoretical oblique SAXS from columnar and no correlation models

6.2 Theoretical oblique SAXS from surface evolution model

6.3 Scattering from a single, oriented ellipsoid

6.4 Schematic of inhomogeneous ellipsoidal particle model

6.5 Spectra of inhomogeneous model compared to data from $\mathrm{Fe}-\mathrm{Ge}$ alloys

6.6 Spectra of inhomogeneous model compared to 6.5 at.\% Mo sample

6.7 Interparticle function in Percus-Yevick approximation for ellipsoids

6.8 Spectia of close-packed ellipsoids compared to 6.3 at.\% Fe sample

6.9 Spectra of close-packed ellipsoids compared to 18.2 at.\% Fe sample

6.10 Spectra of close-packed ellipsoids compared to 18.2 at.\% Fe sample

6.11 Spectra of close-packed ellipsoids compared to 6.3 at.\% Mo sample

6.12 Polydisperse ellipsoid structure factor used to extract interparticle function

6.13 Extracted interparticle interference function from 6.5 at.\% Fe sample

6.14 Gaussian-approximated interparticle interference function

6.15 RDF for Gaussian-approximated interparticle function 


\section{Chapter 1}

\section{Introduction}

For the past several years, vapor-deposited amorphous alloys have been of considerable interest. They can be formed typically over wide composition ranges and, depending on the constituents, have structures that range from continuous random networks ${ }^{1}$ commonly found in covalently bonded amorphous insulators and semiconductors to a dense packing structure ${ }^{2}$ observed in amorphous metals. It is not trivial to predict the structure from the constituents, however, since size differences, chemical bonding, and the kinetics of the deposition process dictate the final structure. Since the amorphous solid is often defined as lacking long-range order, the atomic structure is typically characterized in terms of the high-degree of short-range order. ${ }^{3}$ Most descriptions of vapor-deposited amorphous alloys focus on characterizing this order, while assuming that the material is chemically homogeneous beyond a few near neighbors. Phase separation into regions of different short-range order is, however, endemic in the quasi-equilibrium states of such materials.

This thesis is an attempt to observe and characterize nanoscale phase separation in magnetron sputtered amorphous alloys. The probe used to study the phase separation is anomalous small-angle $\mathrm{x}$-ray scattering (ASAXS), which is sensitive to spatial variations of 
the effective electron density in the material. By coupling the anomalous dispersion relations with traditional SAXS, Rice et al. ${ }^{4}$ detected composition modulations in amorphous metal-germanium alloys, $a-M_{x} G_{1 \cdot x}$ with $\mathrm{M}=\mathrm{Fe}, \mathrm{Mo}$, and $\mathrm{W}$. The work reported in this thesis is a follow-up study to develop a more sophisticated understanding of the reported composition modulations that appear and extend through the metal-insulator transition region $(0<x<0.33)$. Free-standing films (amorphous $\mathrm{Mo}-\mathrm{Ge}$ and $\mathrm{Fe}-\mathrm{Ge}$ ) were grown to remove substrate scattering and oblique transmission measurements recorded to search for anisotropy. This first chapter serves to introduce the reader to the underlying motivation for the work and to provide a background of the alloy's physical properties.

\subsection{Metal-Insulator Transition}

With the addition of metal atoms to the random tetrahedral network 5 of a-Ge, the alloy undergoes several interesting transformations. When co-sputtered with Mo and studied with high-field, low-temperature magnetoresistance, Yoshizumi et al. ${ }^{6}$ show that the electron diffusion constant essentially vanishes when the Mo concentration decreases to approximately 10 at.\%. For co-evaporated samples, Devenyi et al. ${ }^{7}$ noticed that the room temperature resistivity, which changes by 7 orders of magnitude from a-Ge to c-Mo, decreases by $\sim 4$ orders with the addition of only 10 at.\% Mo. They argue that the transition from an insulator to metal occurs near their prepared samples compositions of 7.5 and 16 at.\% Mo.

The resistivity data have not resolved an intriguing question, however. Is the insulator-metal transition well-defined (e.g. is it sharp)? On what parameters does it depend? Specific heat measurements on co-sputtered amorphous Mo-Ge alloys by Mael et al. ${ }^{8}$ indicate that there exists no critical behavior of the thermodynamic electronic density of states, proportional to the linear term in the specific heat, at the metal-insulator transition. They show that a nonvanishing density of states exists throughout the metal-insulator 
transition. Such an observation is consistent with amorphous phase separation into two types of different local structure. The specific heat measurements can be explained if there exist regions in which there are quasi-continuous electron densities of states (i.e. metallic) in a dielectric. In this case, the number of metallic atoms an electron can sample during the measurement time (e.g. via variable range hopping) determine whether or not a quasicontinuum exists.

Similar resistivity results have been reported for co-evaporated amorphous $\mathrm{Fe}-\mathrm{Ge}$ films by Massenet et al. ${ }^{9}$ The change in resistivity at room temperature is -5 orders of magnitude from a-Ge to 10 at.\% Fe, after which the change is only another order of magnitude up to the most Fe-rich sample they studied, 64 at.\% Fe. The conductivity from $20 \mathrm{~K}$ to $300 \mathrm{~K}$ obeys the Mott $T^{1 / 4}$ power law for all films up to 25 at.\% Fe, which is cited as the metal-insulator transition composition. ${ }^{10}$ The metal-insulator transition for the triode-sputtered films made by Lorentz is cited as occurring at 15 at.\% Fe. Depending on the microstructure, others have found quite different critical compositions for the metalinsulator transition. For example, co-evaporated, crystalline In-Ge and Pb-Ge films exhibit a metal-insulator transition near $15 \%$ volume metal atom, whereas co-evaporated, granular Al-Ge films have a much higher critical concentration near $55 \%$ volume Al. ${ }^{11}$

\subsection{Structural Models and Previous Work}

The amorphous Mo-Ge and Fe-Ge systems were chosen for this study, because, as mentioned previously, Rice et al.,4 upon first examination with ASAXS, reported an interesting "composition modulation" in $\mathrm{M}-\mathrm{Ge}$ alloys $(\mathrm{M}=\mathrm{Fe}, \mathrm{Mo}$, and $\mathrm{W})$ that had been speculated by Kortright and Bienenstock ${ }^{12}$ for the Mo-Ge alloys. By working at the accessible $\mathrm{W}$ and $\mathrm{Fe}$ absorption edges, the work illustrates that, for a variety of sputtered M-Ge alloys, the film is not chemically homogeneous beyond a few near-neighbors but exhibits apparent chemical inhomogeneities on a 15-30 $\AA$ size scale (less than $\sim 33$ at.\% 
metal). The chemical inhomogeneity apparently results from a real-space fluctuation of the metal atoms with the density of $\mathrm{Ge}$ atoms constant throughout. They conclude that the composition fluctuations are consistent with regions of local atomic structure that consist of a-Ge and a-MGe $\mathrm{Me}_{2}$, where the Ge density is similar in both (which is the case for crystalline $\mathrm{FeGe}_{2}, \mathrm{MoGe}_{2}$, and $\mathrm{Ge}$ ). Since the Mo K-edge was not accessible at the ti ne, their conclusions are only strictly valid for the Fe-Ge and W-Ge alloys. The follow-up work presented in this dissertation, which emphasizes Mo-Ge through the metal-insulator transition, demonstrates that this alloy is more likely phase-separated into regions of a-Ge and an amorphous intermetallic closer in stoichiometry to a-MoGe $\mathrm{M}_{3}$. The previous work on Mo-Ge and Fe-Ge from Kortright ${ }^{13}$ and Lorentz ${ }^{14}$ focused on large-scale phase separation, so the relevant range in reciprocal space for fine-scale inhomogeneities was not measured. Thus, Rice performed ASAXS measurements on their samples, as well as on W-Ge alloys, to search for a fine-scale inhomogeneity.

The atomic scale structure of Mo-Ge alloys, studied as a function of composition by Kortright, can be classified into three structural regions by composition. In structural region I, from 0 to 23 at.\% Mo, tetrahedral a-Ge coexists on a very fine scale ( $<40 \AA)$ with a Mo-modified structure, most likely resembling the local order of the Ge-rich compounds (e.g. c- $-\mathrm{MoGe}_{2}$ or $\mathrm{c}-\mathrm{Mo}_{13} \mathrm{Ge}_{23}$, the only Ge-rich compounds in the equilibrium phase diagram). At $\sim 23$ at.\% Mo, no evidence of the tetrahedral a-Ge remains, which delimits regions I and II. Region II extends from $\sim 23$ to 50 at.\% Mo, typical of most intermetallic structures (long Mo-Mo distances, strong ordering of Ge around Mo). The collapse of the long Mo-Mo distances leads to region III, with a structure similar to that found in most melt-quenched metal-metalloid glasses.

Kortright performed SAXS to look for phase separation across the composition range of the Mo-Ge alloys but did not observe any indication of large-scale phase separation. In fact, the only appreciable SAXS came from samples with 8 to 15 at.\% Mo, 
which consisted of two contributions. (1) There is a monotonically decreasing signal at very small momentum transfer, whose source remains unknown. The scattering may be possibly from handling the films prior to measurement, cracks, or voids, but it is too weak to arise from phase separation into regions of appreciable difference in electron density. This is in agreement with TEM images that show no deviations from an average structure on this size scale. (2) In addition, there is a weak, diffuse signal whose intensity is of the same order of magnitude as that expected from independent spherical particles with a $5 \AA$ radius. Although the model can be questioned (see Chapter 5), its conclusion is important. The SAXS study indicates that inhomogeneities, on the order of $10 \AA$, may exist. Although not convincing by itself, the SAXS study was supported by other speciesspe:ific experiments (extended $x$-ray absorption fine structure and anomalous large-angle scattering) that upheld this model.

Ding and Anderson ${ }^{15}$ performed molecular-dynamics computer simulations on the structure of Mo-Ge with the primary goal of developing empirical interatomic potentials that lead to structures that agreed with Kortright's scattering measurements. As a result, they noticed that Mo atoms at low atomic percent do not substitute for Ge but rather tend to cluster together, forming chains and rings, and hence distort the local Ge random tetrahedral network, as predicted by Kortright. This is particularly striking, especially since their Mo-Mo two-body potential is the least attractive and the Mo-Ge is most attractive. Since the amorphous state in the simulation cell is formed by rapidly cooling a theoretical "melt" of Mo and Ge atoms, the simulation cannot explain structural effects that depend on the kinetics of the vapor deposition process, however. Although empirical potentials can be found that describe the data of Kortright, any changes in the deposition conditions that lead to changes in the scattering and, hence, structure will require new parameters for each of the interatomic potentials. 
The atomic scale structure of triode-sputtered Fe-Ge alloys has been studied as a function of composition by Lorentz. With the addition of $\mathrm{Fe}$ to a-Ge, the $\mathrm{Ge}$ tetrahedral network becomes significantly altered by 20 at.\% Fe (similar result from the Mo-Ge studies). No large scale phase separation is observed up to a composition of $\mathrm{FeGe}_{2}$, but beyond 33 at.\% Fe, SAXS and $\mathrm{x}$-ray absorption near edge structure (XANES) studies indicate phase separation into a- $\mathrm{FeGe}_{2}$ and $\mathrm{a}-\mathrm{Fe}_{3} \mathrm{Ge} .{ }^{16}$ Rice performed ASAXS for this composition range on the samples previously studied with normal SAXS by Lorentz, and the data are currently being reduced and interpreted. These samples exhibit density fluctirations on a much larger size scale and persist up to but not including the most Fe-rich sample ( 72 at.\% Fe) made by Lorentz. As mentioned previously, Lorentz did not look for a fine scale phase separation for $0<x<0.33$ but could not dismiss it either. Rice proceeded to observe the apparent composition fluctuations in this composition range with ASAXS.

In addition to Rice's work, my literature review has indicated that ASAXS has been used to study only two other vapor-deposited amorphous alloys. Maret et al. ${ }^{17}$ have studied sputtered $\mathrm{Tb}-\mathrm{Cu}$ and $\mathrm{Gd}-\mathrm{Cu}$ alloys with ASAXS, which give similar scattering features as that reported by Rice (with a well-defined SAXS maximum at $k-0.2 \AA^{-1}$ ). Their results are consistent with phase separation into rare-earth hydride particles (hydrogen incorporated in the film in the fabrication process) and a rare-earth poor matrix.

\subsection{New Interests}

The goal of the thesis is to understand on a more quantitative and detailed level the apparent composition fluctuations that have been observed. Such fluctuations have not been reported before for vapor-deposited amorphous alloys, and they represent a new class of materials which have otherwise always been considered homogeneous down to a few near-neighbors. In particular, we sought to answer the following questions. 
(1) What is a likely real-space description of the source of the scattering? Since the alloy is vapor deposited with its lateral dimensions and growth direction fixed, one could imagine phase separation that is not isotropic. Is there any anisotropy present in these films? In fact, Cargill ${ }^{18}$ noticed a strong anisotropy in evaporated a-Ge with SAXS, which has been interpreted as the scattering from rod-like voids oriented in the direction of growth ( $\sim 30 \AA$ in-plane by $\sim 2200 \AA$ growth direction). Similar structural characteristics are observed in some electrodeposited amorphous Gd-Co alloys ${ }^{19}$ and sputtered and evaporated $\mathrm{Ni}-\mathrm{Fe}$ alloys ${ }^{20}$ that are, in some cases, related to the observed large magnetic anisotropies. The ASAXS studies of Rice ${ }^{4}$ and Maret et al. ${ }^{18}$ were performed in normal transmission, with the scattering vector in the plane of the sample. To search for anisotropy, the orientation of the scattering vector must vary with the film surface; that is, with it oblique to and in the plane of the films. The experiment designed to test for the anisotropy is outlined in Chapter 3. Since correlations in electron density are projected onto the line defined by the scattering vector, interpretation of the scattering results is difficult. To interpret the anisotropy that we did, in fact, observe, we utilized the cylindrical correlation function and constructed particle models that might resemble, on an averaged, simplified scale, an anisotropic phase separation. The anisotropic scattering and correlation function results are presented in Chapters 4 and 5 for amorphous $\mathrm{Fe}-\mathrm{Ge}$ and Mo-Ge, respectively, and Chapter 6 reports on model structures.

(2) What is an accurate estimate of the overall magnitude of the composition fluctuations? If there are regions of well-defined electron density (particles), what are the compositions of the phase-separating species? With an adequate sampling of the anisotropic scattering, the total cross-section can be determined which is proportional to the mean-square fluctuation of electron density. When compared with that expected from twoand three-phase models, we can determine the composition of the phase-separating species, and we have also explored the reliability of the approach developed. These ideas are 
examined in detail in the Chapters which discuss the Fe-Ge and Mo-Ge results (Chapters 4 and 5).

(3) To what extent can we modify the degree and/or extent of phase separation by varying the deposition conditions? With such high quench rates, the vapor deposition process often leads to atomic arrangements that depend on the method of preparation. With annealing at elevated temperatures, for example, annihilation of excess free volume can lead to an amorphous structure with atomic arrangements that are more favorable energetically. ${ }^{21}$ Hence, the growth parameters (e.g. growth rate, power to targets, samplesubstrate distance, etc.) are likely to be the most defining characteristic of the films other than its chemical constituents. On a very simple level, we tried to observe any changes in the SAXS spectrum, for a given Fe concentration, simply by changing the deposition conditions. This is explored in Chapter 4.

(4) Are the amorphous Mo-Ge films truly phase-separated? That is, are the Mo atoms distributed inhomogeneously as suggested by Rice's work at the Ge K-edge for the Mo-Ge system? What limited Rice et al. experimentally and how can the next set of experiments overcome these problems? Rice was unable to collect Mo K-edge anomalous scattering data and addresses the experimental problem in her dissertation. Chapter 3 discusses collection of ASAXS data at hard $\mathrm{x}$-ray energies and how we overcame the problems of Ge fluorescence and poor detector efficiency. Another major limitation has been a poor signal-to-noise ratio (SNR) with kapton-supported films. Since the experiments are performed in transmission, scattering from kapton often overwhelms the signal. In some cases, it is only after subtraction of the kapton that the weak scattering from the amorphous films is even evident. This is a major problem. In fact, an anisotropy study performed in August, 1991, with kapton-supported films led to scattering that was so overwhelmingly swamped with kapton background that it was difficult to interpret the anisotropy on a quantitative level. In addition, kapton itself is a very anisotropic 
scatterer, ${ }^{22}$ so the removal of all substrate scattering is a necessity. Films that could be grown and rendered free-standing were made. Details of the sample preparation and initial characterization are outlined in Chapter 2. 


\section{References for Chapter 1}

${ }^{1}$ W.H. Zachariasen, J. Am. Chem. Soc. 54, 3841 (1932).

2J.D. Bernal, Nature 183, 141 (1959).

3R. Zallen, The Physics of Amorphous Solids (John Wiley \& Sons, New York, 1983).

${ }^{4}$ M. Rice, S. Wakatsuki, and A. Bienenstock, J. Appl. Cryst. 24, 598 (1991).

5D.E. Polk, J. Non-Crystalline Solids 5, 365 (1971).

${ }^{6}$ S. Yoshizumi, T.H. Geballe, M. Kunchur, and W.L. McLean, Phys. Rev. B 37, 7094 (1988).

7A. Devenyi, C. Rusu, A. Belu, and R. Manaila, J. De Phys. Colloque 42, C4-1089 (1981).

8D. Mael, S. Yoshizumi, and T.H. Geballe, Phys. Rev. B 34, 467 (1986).

${ }^{9}$ O. Massenet, H. Daver, and J. Geneste, J. De Phys. Colloque 35, C4-279 (1974).

${ }^{10} \mathrm{H}$. Daver, O. Massenet, and B. Chakraverty, Proc. Fifth Intl. Conf. Amorphous and Liquid Semiconductors, Garmish, 1973, p. 1053.

${ }^{11}$ G. Deutscher, M. Rappaport, and Z. Ovadyahu, Solid State Comm. 28, 593 (1978).

12J.B. Kortright and A. Bienenstock, Phys. Rev. B 37, 2979 (1988).

13J.B. Kortright, Ph.D. Thesis, Stanford University (1984).

${ }^{14}$ R.D. Lorentz, Ph.D. Thesis, Stanford University (1986).

${ }^{15}$ K. Ding and H.C. Anderson, Phys. Rev. B 36, 2675 (1987).

16R.D. Lorentz, A. Bienenstock, and T.I. Morrison, accepted for publication in Phys. Rev. B (1994).

${ }^{17}$ M. Maret, J.P. Simon, B. Boucher, R. Tourbot, and O. Lyon, J. Phys.: Condens. Matter 4, 9709 (1992).

18G.S. Cargill, Phys. Rev. Lett. 28, 1372 (1972). 
${ }^{19}$ T. Mizoguchi and G.S. Cargill, J. Appl. Phys. 50, 3570 (1979).

${ }^{20}$ G.S. Cargill, S.R. Herd, W.E. Krull, and K.Y. Ahn, IEEE Trans. Magn., Vol. Mag15,1821 (1979).

${ }^{21}$ E.P. Donovan, F. Spaepen, D. Turnbull, J.M. Poate, and D.C. Jacobsen, J. Appl. Phys. 57, 1795 (1985).

22R.F. Boehme and G.S. Cargill in Polyimides, Vol. 1, ed. by K.L. Mittal (Plenum Publishing, 1984), 461. 


\section{Chapter 2}

\section{Sample Preparation and Characterization}

The atomic arrangements in sputtered amorphous films depend to a large degree on the kinetics of the deposition process. ${ }^{1}$ This is a result of the inter-relationship between the high "quench" rates that can be obtained and the very short time that is available for diffusion and chemical bonding. The sample preparation procedure thus plays an integral role in the atomic arrangements found in each film. Since several students ${ }^{2}$ have made amorphous binary alloys from the 3" system at the Vapor Phase Synthesis Laboratory of the Center for Materials Research (CMR) at Stanford, only a summary of the sputtered samples will be given here. The reader is also referred to the work of Wilson ${ }^{3}$ who describes in detail recipes to make free-standing films and who outlines the arguments for thickness and atomic percent calculations using $\mathbf{x}$-ray absorption measurements.

\subsection{Sample Preparation}

Amorphous $\mathrm{Fe}_{x} \mathrm{Ge}_{1 \cdot x}$ and $\mathrm{Mo}_{x} \mathrm{Ge}_{1 \cdot x}$ alloys were prepared at room temperature by magnetron co-sputtering of elemental targets onto a rapidly rotating substrate table (300 $\mathrm{rpm}$ ), so that only a fraction of a monolayer is deposited as the sample is rotated in front of one target and then the next. The sputtering geometry was in the vertical, with the substrate 
table always positioned 3" below the planar magnetron sputter guns. The base pressure of the chamber was never higher than $210^{-6}$ torr, while at times as low as $710^{-7} \mathrm{Torr}$, and the sputtering gas, purified Ar, always held at 2 mTorr. A summary of the different deposition conditions, targets used, gun settings, film characteristics, etc., can be found in table 2.1.

The Ge and Mo targets were typically $2 "$ in diameter by $1 / 8$ " thick, while the $\mathrm{Fe}$ targets were always $2 "$ by $0.080 "$. Thicker Fe targets could not be used because the permanent magnets at CMR were not strong enough to compensate for the Fe target's high permeability. In the previous study by Lorentz, the Fe was triode-sputtered, since the strong permanent magnets that were used in this work were not yet available at CMR. Magnetron sputtering is favored over the triode-sputtering method, since the magnetron does a much better job of electron confinement. ${ }^{4}$ This increases the efficiency of the system, permitting us to work at low voltages and low gas pressures. With high gas pressures, collisions of the sputtered atoms with the sputter gas lead to a broad energy distribution and lower mean energy of the adatoms ("thermalization"5). One other advantage with the magnetron system is that, with greater electron confinement, it is possible to limit the bombardment of the emerging film with electrons and ions. However, the reduced gas scattering can lead to bombardment of the emerging film by the adatoms themselves. ${ }^{6}$

The Ge RF power was usually set in the range of $350-400$ watts (except for some samples grown at $\sim 100$ watts--see table 2.1 ), while the Mo or Fe DC current was varied from $0.02-0.20$ amps to control the overall composition of the film (for $x<0.33$ ). Some Fe-Ge samples were prepared using different target power and current settings. However, it is difficult to grow thick samples of identical composition under various conditions with the 3" system at CMR. The sputtering rates change slightly as a function of time, being dependent on the target thickness; to grow a sample of a particular composition is often a 'hit' or 'miss'. Although useful, the rate tests (deposition rate as a function of power or 
current setting) mentioned by others (e.g. Kortright, Wilson) are simply too crude to obtain films of accurate composition from one deposition to another. Fortunately, enough samples have been grown that similar Fe-Ge compositions have been obtained for films grown under various power settings.

The substrates consisted of $\mathrm{Si}(100)$ wafers, glass microscope cover slips and slides, and $0.001 "$ kapton. The Si wafers were cleaned with standard semiconductor methods, the glass slides by acetone and methanol, and the kapton by soap and deionized water and blown with dry $\mathrm{N}_{2}$ gas. Film growth rates varied from 1-4 $\AA / \mathrm{sec}$ depending on target power settings, but typically 5-10 micron thick films could be grown in 7-10 hours. With a table rotation of $300 \mathrm{rpm}$, approximately 0.2 to $0.8 \AA$ of material are deposited for each revolution under the sputter guns. The films were grown to such thicknesses that they could support their own weight and not crack or flake once the Si support was removed. For films thicker than 6-7 microns, the Si substrates could be removed by immersion in a warm $\mathrm{KOH}$ bath for approximately 4 hours. The details of the etching procedure are described by Wilson. ${ }^{3}$ Samples that are sufficiently thick and free-standing are $6.3,10.8$, 13.6, and 18.2 at.\% $\mathrm{Fe}$ and $6.5,12.4,16.4$, and 23.6 at.\% Mo; the rest of the samples (listed in table 1) are only kapton- and glass-supported.

\subsection{Initial Characterization}

Due to the previous work of Kortright and Lorentz, who have searched amorphous alloys of similar composition and preparation methods for crystallization and contamination, little more than a visual examination was required. After sputtering, the films are very smooth with a shiny surface. In some isolated cases, there exist large areas of cracks and flakes, where the material has crystallized. These are most evident in samples grown for long times and at the higher $\mathrm{Fe}$ and Mo atomic fractions. $\mathrm{A}$ conventional Picker diffractometer was used to insure that the samples used in this study 
had not crystallized. Moreover, since there was no etch stop protecting the films, there was concern that the Si substrate removal procedure with $\mathrm{KOH}$ may have damaged the films. There was no visual evidence for such damage, however, and the Picker scans showed no change between the free-standing films and those that were kapton-supported. These observations are in agreement with Wilson, who also observed no change in the Mo-Ge alloy with $\mathrm{KOH}$ etch. Additionally, the ASAXS patterns show no difference between the free-standing and kapton-supported films (from the same deposition), except for the farimproved SNR.

The kapton-supported films often curled, with the deposited film on the outside. For films of the same atomic percent metal, the curling is greater the thicker the film, and for films of the same thickness, greatest curling occurs for a-Ge. Such observations had been reported earlier by Kortright, ${ }^{2}$ who argued that the "curling presumably results from different thermal expansions of the films and kapton on cooling...it is well known that tetrahedral semiconductors have small thermal expansion coefficients compared to metals, and this is consistent with the observed curling behavior..." (p. 35). The curling effect has not been pursued beyond these observations and was not evident in the free-standing films. This tends to support Kortright's idea that the thermal expansion between the kapton and film is the cause of the curling phenomenon.

An extra glass slide was also attached to the substrate table, with a small region $-1 \mathrm{~cm}^{2}$ masked. After the deposition with the mask removed, an alpha-step profilometer was used to determine the film thickness. The profilometer uses a diamond-tipped stylus to mechanically measure the height difference between the masked and unmasked regions. Finally, an electron microprobe was used to determine the atomic percent metal in each alloy. 


\subsection{X-Ray Absorption Measurements}

To take advantage of differential anomalous scattering, the atomic scattering factors (ASFs) near the relevant atomic absorption edges must be determined. The ASF, $f(k, E)$, is defined as the ratio of the amplitude of coherent scattering from an isolated atom to that from a free electron. It is a function of the photon energy $E$ and the scattering vector $k=4 \pi \sin \theta / \lambda$, with $2 \theta$ the scattering angle. At small $k$, the ASFs are essentially constant for a given $\mathrm{x}$-ray energy. Since we focus only on scattering in this small-angle region and there is little if any noticeable change in the scattering factor with angle in this region, we have ignored any angular dependence in the ASF and write it simply as $f(E)$.

Near an absorption edge an anomalous dispersion effect occurs, and one must include the anomalous dispersion corrections to the total scattering factor:

$$
f(E)-Z+f^{\prime}(E)+i f^{\prime \prime}(E)
$$

with $Z$ the number of electrons in the atom and $f^{\prime}(E)$ and $f^{\prime \prime}(E)$ the real and imaginary components, respectively. The imaginary component is directly proportional to the atom's absorption cross-section by the optical theorem. The real component of the ASF is then determined by the Kramers-Kronig dispersion relation. For energies lower in value and just below the absorption edge, $f^{\prime \prime}(E)$ is essentially constant and $f^{\prime}(E)$ is increasingly negative as the edge is approached. Above the edge, $f^{\prime \prime}(E)$ is much larger, with the sample absorbing photons more readily with the photoelectric effect. The variation of the ASF with energy permits a variable contrast effect that allows one to highlight a particular element's contribution to the total scattering.

Different methods for determining the ASFs have been critiqued by Fuoss, ${ }^{7}$ and the one chosen for this work is conceptually the simplest. The absorption cross-section for a 
particular atomic species is determined by placing an ionization chamber before the sample (monitor incident intensity) and one after the sample (monitor forward scattered intensity), scanning in energy through an accessible absorption edge (e.g. Fe, Ge, and Mo K-edge), and subtracting the absorption cross-section contribution from other sources. This is the standard EXAFS experiment using two ion chambers in a forward-scattering geometry. After removing the background and absorption from other elements in the sample, the absorption data are placed on an absolute scale by normalizing the data to the free-atom ASF given by the Cromer-Liberman values. Then, with the aid of the optical theorem and the Kramers-Kronig integral, the energy dependent ASFs are extracted.

In practice, the ASFs were obtained as follows. Absorption edge data sets were taken at the eight-pole focused wiggle: side-station 4-1 of the Stanford Synchrotron Radiation Laboratory (SSRL) on all of the sputtered films. By scanning through the Fe, $\mathrm{Ge}$, and Mo K-edges (see Section 3.3 for a discussion of the $\mathrm{x}$-ray optics), the absorption cross-section for different samples and energies have been measured. The background and absorption from other species as well as a changing detector function is removed with a $1 / E$ cubic polynomial. The EXAFS region, which is structure sensitive and thus sample specific, is excluded from this fit. Then, the absorption data are placed on an absolute scale by normalizing the data far from the edge to the free-atom ASF with Cromer-Liberman values. FORTRAN program FPPFIT.FOR, written by Wilson from an earlier version of a program from Ludwig, ${ }^{8}$ performs the above subtraction and normalization procedure and then determines $f^{\prime \prime}(E)$ from the optical theorem. Program FPCL.FOR by Ludwig calculates the $f^{\prime}(E)$ by evaluating the Kramers-Kronig integral..$^{9}$ The ASFs for all freestanding samples were determined. Figure 2.1a shows the raw data at the Ge K-edge for one of the samples, 23.6 at.\% Mo, prior to background subtraction and data normalization. Figure $2.1 \mathrm{~b}$ and $2.1 \mathrm{c}$ illustrate the extracted $f^{\prime}(E)$ and $f^{\prime \prime}(E)$ for this sample using the above mentioned procedure and computer programs. 

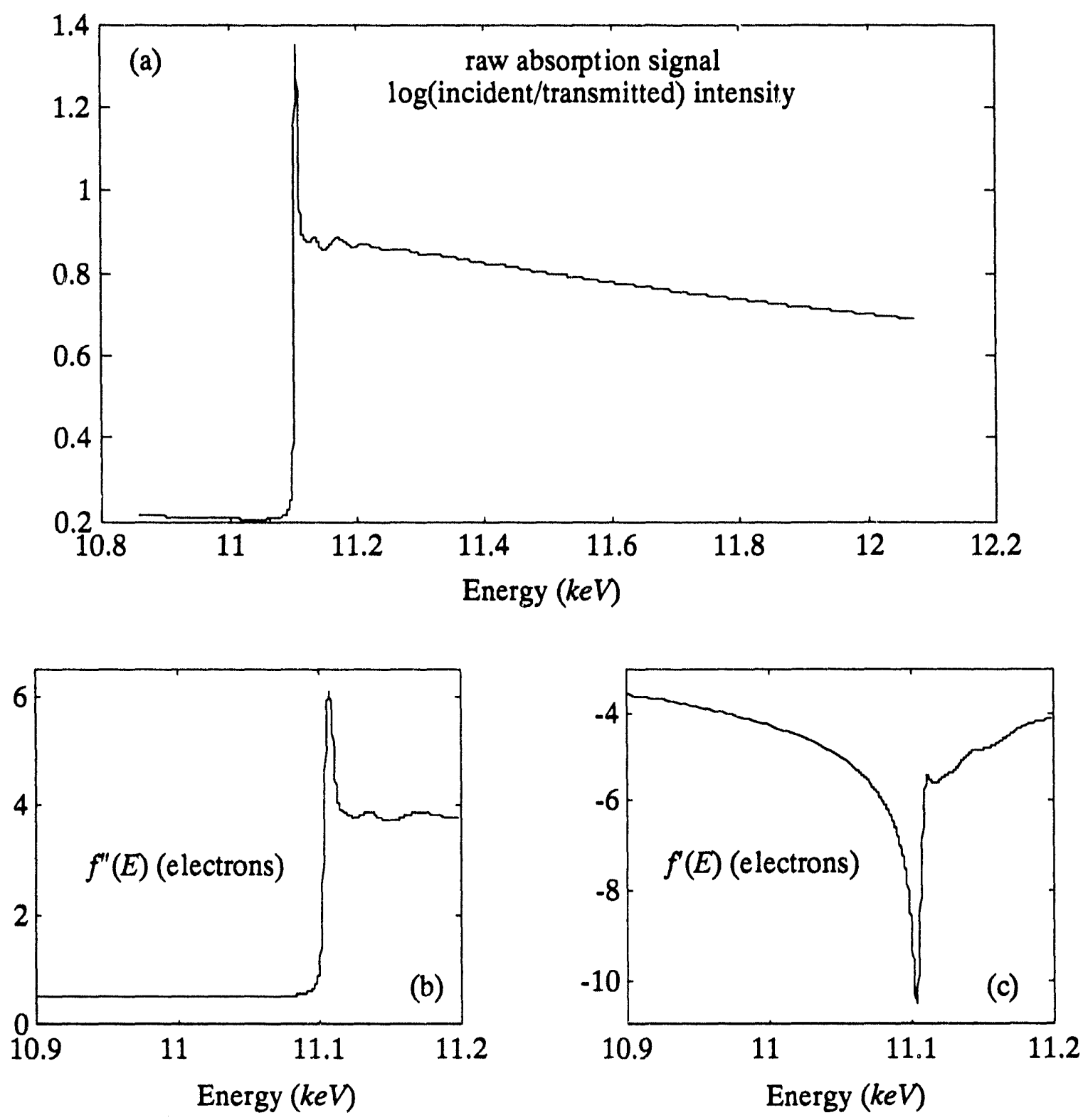

Figure 2.1. (a) Raw absorption data at the Ge K-edge for one of the samples, 23.6 at.\% Mo, prior to background subtraction and data normalization. (b) and (c) illustrate the extracted Ge $f^{\prime}(E)$ and $f^{\prime \prime}(E)$ for this sample. 
The scale factor that relates the absorption cross-section to the Cromer-Liberman value provides a measure of the number density of the atomic species in the material. This has lead to a method that can be used to determine the composition of the film (see Wilson) using $\mathrm{x}$-ray absorption measurements. For absorption measurements at the $\alpha$ edge, the scale factor is equal to the atomic number density of element $\alpha$ multiplied by the total thickness $t$ of the film, $n_{\alpha} t$. For a binary alloy, an accurate determination of both $n_{A} t$ and $n_{B} t$ allows the atomic fraction $\mathrm{A}$ in $\mathrm{A}_{x} \mathrm{~B}_{1 \cdot x}$ to be calculated:

$$
x=\frac{n_{A} t}{n_{A} t+n_{B} t} .
$$

By assuming an average number density for the overall film, $n_{\text {avg }}$, the thickness can be calculated, $t=\left(n_{A} t+n_{B} t\right) / n_{\text {avg }}$. For a given film of metal concentration $\mathrm{x}(\mathrm{x}<0.33), n_{\text {avg }}$ was taken as $95 \%$ of the weighted average number densities of $\mathrm{c}-\mathrm{Ge}$ and $\mathrm{c}-\mathrm{MGe}_{2}(\mathrm{M}=\mathrm{Mo}$, $\mathrm{Fe}$ ). Table 2.1 compares the compositions and thicknesses for the different alloys with the alpha-step and electron microprobe results. 
Table 2.1 Summary of deposition conditions and characteristics for samples studied.

\begin{tabular}{|c|c|c|c|c|c|c|}
\hline $\begin{array}{l}\text { sample } \\
\text { (alloy) }\end{array}$ & substrates & $\begin{array}{l}\mathrm{Fe} \text { or Mo } \\
\text { (dc--amps) }\end{array}$ & $\begin{array}{c}\text { Ge gun } \\
\text { (rf--watts) }\end{array}$ & $\begin{array}{l}\text { deposition } \\
\text { time }\end{array}$ & $\begin{array}{l}\text { at. } \% \text { metal }^{a} \\
\text { e.m./edge }\end{array}$ & $\begin{array}{l}\text { thickness }^{b} \\
\alpha \text {-step/edge }\end{array}$ \\
\hline $\begin{array}{l}89285 \\
\mathrm{Fe}-\mathrm{Ge}\end{array}$ & $\begin{array}{l}\text { kapton, } \\
\text { glass }\end{array}$ & 0.043 & 400 & $2.1 \mathrm{hrs}$ & 10.7 & $1.00 \mu \mathrm{m}$ \\
\hline $\begin{array}{c}89286 \\
\text { Fe-Ge }\end{array}$ & $\begin{array}{c}\text { kapton, } \\
\text { glass }\end{array}$ & 0.022 & 139 & 4.2 & 8.7 & 1.15 \\
\hline $\begin{array}{l}89287 \\
\text { Fe-Ge }\end{array}$ & $\begin{array}{c}\text { kapton, } \\
\text { glass }\end{array}$ & 0.322 & 400 & 1.5 & $\begin{array}{l}45.11 \\
43.86\end{array}$ & $\begin{array}{c}0.975 / \\
1.06\end{array}$ \\
\hline $\begin{array}{c}89288 \\
\mathrm{Fe}-\mathrm{Ge} \\
\end{array}$ & $\begin{array}{l}\text { kapton, } \\
\text { glass }\end{array}$ & 0.119 & 100 & 3.5 & $\begin{array}{l}48.21 \\
46.64 \\
\end{array}$ & $\begin{array}{c}0.7601 \\
0.843 \\
\end{array}$ \\
\hline $\begin{array}{r}89289 \\
\text { Fe-Ge } \\
\end{array}$ & $\begin{array}{l}\text { kapton, } \\
\text { glass }\end{array}$ & 0.059 & 400 & 2.25 & 23.9 & 1.38 \\
\hline $\begin{array}{r}89290 \\
\mathrm{Fe}-\mathrm{Ge}\end{array}$ & $\begin{array}{l}\text { kapton, } \\
\text { glass }\end{array}$ & 0.024 & 100 & 3.5 & 11.5 & 0.725 \\
\hline $\begin{array}{l}89291 \\
\mathrm{Fe}-\mathrm{Ge} \\
\end{array}$ & $\begin{array}{c}\text { kapton, } \\
\text { glass }\end{array}$ & 0.154 & 400 & 2.0 & 25.1 & 1.30 \\
\hline $\begin{array}{c}89292 \\
\mathrm{Fe}-\mathrm{Ge} \\
\end{array}$ & $\begin{array}{c}\text { kapton, } \\
\text { glass }\end{array}$ & 0.059 & 100 & 4.0 & 27.0 & 0.875 \\
\hline $\begin{array}{c}89293 \\
\mathrm{Fe}-\mathrm{Ge} \\
\end{array}$ & $\begin{array}{l}\text { kapton, } \\
\text { glass }\end{array}$ & 0.028 & 400 & 2.25 & 5.8 & 1.33 \\
\hline $\begin{array}{c}89294 \\
\mathrm{Fe}-\mathrm{Ge} \\
\end{array}$ & $\begin{array}{l}\text { kapton, } \\
\text { glass }\end{array}$ & 0.011 & 100 & 3.5 & 6.2 & 0.715 \\
\hline $\begin{array}{l}89304 \\
\mathrm{Mo}-\mathrm{Ge} \\
\end{array}$ & $\begin{array}{l}\text { kapton, } \\
\text { glass, Si }\end{array}$ & 0.06 & 400 & 11.0 & $\begin{array}{l}6.31 \\
6.53 \\
\end{array}$ & $\begin{array}{c}9.00 / \\
9.89 \\
\end{array}$ \\
\hline $\begin{array}{l}89305 \\
\text { Mo-Ge }\end{array}$ & $\begin{array}{l}\text { kapton, } \\
\text { glass, Si }\end{array}$ & 0.16 & 400 & 4.0 & $\begin{array}{l}16.4 ! \\
16.51 \\
\end{array}$ & $\begin{array}{c}3.901 \\
4.22\end{array}$ \\
\hline $\begin{array}{l}89306 \\
\text { Mo-Ge }\end{array}$ & $\begin{array}{l}\text { kapton, } \\
\text { glass, Si }\end{array}$ & 0.11 & 400 & 10.0 & $\begin{array}{l}11.6 / \\
12.41 \\
\end{array}$ & $\begin{array}{l}7.70 / \\
8.46 \\
\end{array}$ \\
\hline $\begin{array}{c}89307 \\
\mathrm{Fe}-\mathrm{Ge}\end{array}$ & $\begin{array}{l}\text { kapton, } \\
\text { glass, Si }\end{array}$ & 0.046 & 400 & 6.5 & $\begin{array}{l}6.21 \\
6.27 \\
\end{array}$ & $\begin{array}{c}6.401 \\
6.85 \\
\end{array}$ \\
\hline $\begin{array}{l}89308 \\
\mathrm{Fe}-\mathrm{Ge}\end{array}$ & $\begin{array}{l}\text { kapton, } \\
\text { glass, Si }\end{array}$ & 0.06 & 400 & 10.0 & $\begin{array}{l}10.01 \\
10.80 \\
\end{array}$ & $\begin{array}{l}8.201 \\
8.21 \\
\end{array}$ \\
\hline $\begin{array}{r}89309 \\
\mathrm{Fe}-\mathrm{Ge} \\
\end{array}$ & $\begin{array}{l}\text { kapton, } \\
\text { glass, } \mathrm{Si}\end{array}$ & 0.10 & 400 & 6.5 & $\begin{array}{l}12.8 I \\
13.62 \\
\end{array}$ & $\begin{array}{c}7.001 \\
6.99 \\
\end{array}$ \\
\hline $\begin{array}{l}89310 \\
\text { Mo-Ge } \\
\end{array}$ & $\begin{array}{l}\text { kapton, } \\
\text { glass, Si }\end{array}$ & 0.21 & 400 & 9.0 & $\begin{array}{l}21.41 \\
23.62 \\
\end{array}$ & $\begin{array}{l}7.301 \\
7.74 \\
\end{array}$ \\
\hline $\begin{array}{r}89311 \\
\mathrm{Fe}-\mathrm{Ge} \\
\end{array}$ & $\begin{array}{l}\text { kapton, } \\
\text { glass, Si }\end{array}$ & 0.14 & 400 & 6.0 & $\begin{array}{l}17.9 / \\
18.19 \\
\end{array}$ & $\begin{array}{c}6.10 / \\
6.55 \\
\end{array}$ \\
\hline
\end{tabular}

a The atomic percent metal in each of the samples has been determined for all samples with the electron microprobe (e.m.). In some cases, the absorption method (edge) has also been used to determine the metal concentrations (separated by ' $/ /$ ). When measured, we usually cite the edge method as giving a more accurate metal concentration, since it samples the entire film thickness whereas the e.m. probes only the top $1000-4000 \AA$.

${ }^{b}$ The thickness of each sample from the sputtering runs are measured with the alpha-step profilometer ( $\alpha$-step). In some cases (separated by ' $/$ ), the thickness has also been measured by absorption methods [with an assumed average number density of atoms in the film (Eq. 2.3.2)]. 
References for Chapter 2

1T.W. Barbee and D.L. Keith in Synthesis and Properties of Metastable Phases, ed. by E.S. Machlin and T.J. Rowland, Proc. Metall. Soc. of AIME, Pittsburgh, October 5-9, 1980. See also A.K. Sinha, B.C. Giessen, and D.E. Polk in Treatise on Solid State Physics, ed. by N.B. Hannay (New York: Plenum, 1976).

2J.B. Kortright, Ph.D. Thesis, Stanford University (1984). R.D. Lorentz, Ph.D. Thesis, Stanford University (1987).

3L.C. Wilson, Ph.D. Thesis, Stanford University (1990).

${ }^{4}$ R. Parsons in Thin Film Processes II, ed. by J.L. Vossen and W. Kern (Academic Press, Inc., San Diego, 1991).

${ }^{5 K}$. Meyer, I.K. Schuller, and C.M Falco, J. Appl. Phys. 52, 5803 (1981).

${ }^{6}$ D.W. Hoffman and J.S. Badgley, J. Vac. Sci. Technol. A 5, 1791 (1987).

7P.H. Fuoss, Ph.D. Thesis, Stanford University (1980).

${ }^{8}$ K.F. Ludwig, Ph.D. Thesis, Stanford University (1986).

9J.J. Hoyt, D. DeFontaine, and W.K. Warburton, J. Appl. Cryst. 17, 344 (1984). 


\section{Chapter 3}

\section{ASAXS Experiment and Data Treatment}

In this chapter the basic relationships between differential anomalous scattering and the structure factor and pair correlation functions are established. In addition, the experimental methods used to obtain these functions will be outlined in detail.

\subsection{Structure Factor and Pair Correlation Functions}

In a typical $x$-ray scattering experiment, the measurable quantity is the differential cross-section $d \sigma / d \Omega$ of scattered radiation, consisting of the Thomson, photoelectric, Compton, and, if near atomic absorption edges, resonant-Raman contributions. Elastic or coherent scattering occurs when energy is conserved in a scattering event and is often referred to as the Thomson cross-section, $d \sigma_{\text {elas }} / d \Omega$. It is this quantity that is most often used to determine atomic arrangements in materials. In the photoelectric effect, a photon is absorbed by an atom which leads to the ejection of an electron to the continuum and emission of additional, lower energy photons from the atom (fluorescence). Similarly, resonant-Raman scattering, ${ }^{1}$ which is appreciable only for $\mathbf{x}$-ray energies just below atomic absorption edges, results from the following inelastic process. An incoming photon, for example with energy just less than an atom's $\mathrm{K}$ electron, is absorbed by the atom which 
leads to a transition from the initial ground state to a virtual state and then to a final state. In the final state, the atom is left with a hole in the $\mathrm{L}$ shell, and a photon is emitted with a lower energy than the initial one. Thcse lower energy photons (fluorescence and resonant Raman scatter) are distributed over $4 \pi$ solid angle and are detected as constant background superimposed on the coherent scattering. Compton scattering, another inelastic or incoherent process, is negligible at small angles and is not considered in the analysis.

The knowledge of the differential cross-section provides an immense amount of information about electron density correlations in the material. With the kinematical theory of $\mathbf{x}$-ray scattering, the differential cross-section that arises from the elastic $\mathbf{x}$-ray scattering is proportional to the absolute square of the Fourier transform of the sample's effective electron density, $\rho(\bar{x}, E)$ :

$$
\frac{d \sigma_{e l a s}}{d \Omega}(\vec{k}, E)=r_{e}^{2}\left|\int_{V} \rho(\bar{x}, E) e^{-i \bar{k} \cdot \bar{x}} d^{3} x\right|^{2}
$$

with $r_{e}=\frac{e^{2}}{m c^{2}}=2.817910^{-5} \AA$, the Thomson coefficient for the scattering amplitude for a free electron (also known as the classical radius of the electron), and $V$ the scattering volume for photons of energy $E$ (wavelength $\lambda$ ). The differential cross-section is a function of photon energy and momentum transfer $\vec{k}$, defined in direction by the incident and scattered wavevectors and in magnitude as $4 \pi \sin \left(\frac{2 \theta}{2}\right) / \lambda$, with $2 \theta$ the angle between the incident and scattered photons. To isolate the elastic term in Eq. 3.1.1, the contribution of the inelastic processes must be removed from the total, measured differential cross-section. This will be discussed in more detail in Section 3.6.

The effective electron density is a function of the wavelength that is used to measure the differential cross-section. Since the experiment is performed at small angles, the volume elements that are probed are large compared to the typical atomic volumes in the 
sample, and thus each atom in the volume element contributes a certain number of electrons. At energies far removed from any absorption edges in the material, each atom in the volume element contributes a number of electrons equal to its atomic number. For energies near an absorption edge of an atom in the material, the anomalous scattering factors will define the effective electron density. In particular, the electron density at a particular point $\bar{x}$ (with respect to an arbitrary reference frame) is the sum of the product of the number density for a particular atomic specie $\left(n_{\alpha}\right)$ by its effective number of scattering electrons, summed over all species in the material:

$$
\rho(\vec{x}, E)=\sum_{\alpha} n_{\alpha}(\vec{x})\left|Z_{\alpha}+f_{\alpha}^{\prime}(E)+i f_{\alpha}^{\prime \prime}(E)\right|
$$

In amorphous materials, the differential cross section is usually assumed isotropic; that is, $d \sigma / d \Omega$ is a function of $k$ only. In this work, the differential cross section and, hence, the associated structure factors and pair correlation functions are strongly anisotropic and are expressed as a function of $\vec{k}$ explicitly. Figure 3.1 illustrates the polar coordinate system with respect to the film surface normal that is used in this work. Notice that the polar coordinates are $\left(k, \theta_{k}, \phi_{k}\right)$, with $2 \theta$ the angle between the incident and scattered photon and not related to the polar angle coordinate $\theta_{k}$. In order to remove sample and experimental parameters from the differential cross-section, it is convenient to define a function called the structure factor, $S(\vec{k}, E)$, that is proportional to the elastic contribution of the differential cross-section per unit volume of material:

$$
S(\vec{k}, E)=r_{e}^{-2} V^{-1} \frac{d \sigma_{e l a s}}{d \Omega}(\vec{k}, E)
$$




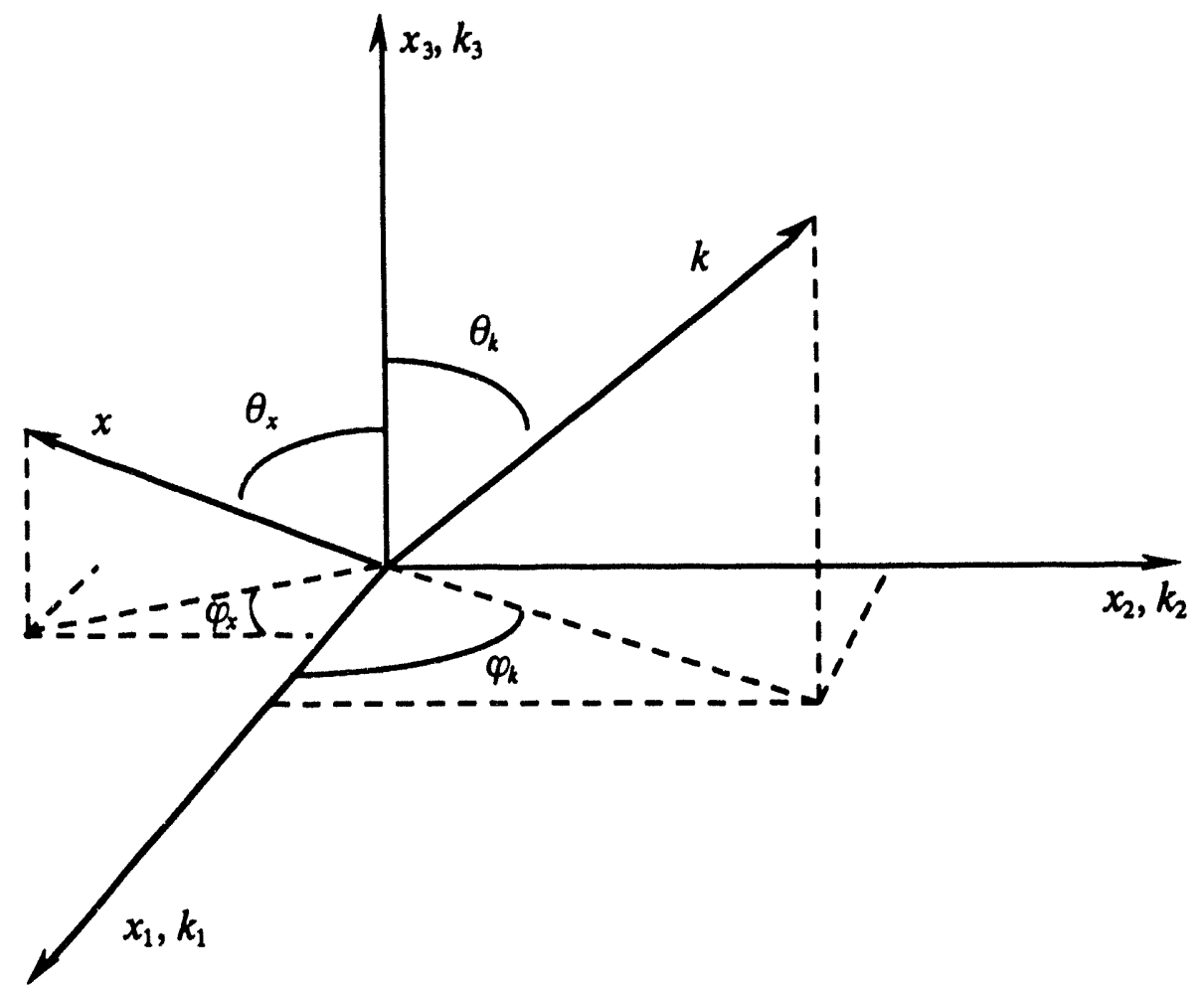

Figure 3.1 Spherical coordinate system used in this work. The sample is in the $x_{1}-x_{2}$ plane, with $x_{3}$ in the direction of growth. 
The structure factor has dimensions of (electrons) ${ }^{2}$ per volume and is simply the absolute square per unit volume of the effective electron density Fourier transform.

The origin of the $\mathbf{x}$-ray scattering is not from the electron density itself; rather, from any changes in electron density (this is of course not true if forward scattering is included). To understand this in the context of the above equations, define the electron density difference $\eta(\bar{x}, E)$ as the difference between the electron density at $\bar{x}$ and the mean density, $\rho_{0}(E)$. That is, $\eta(\bar{x}, E)=\rho(\bar{x}, E)-\rho_{0}(E)$. Inserting this into Eq. 3.1.1 with Eq. 3.1.2 yields

$$
S(\bar{k}, E)=\frac{1}{V}\left|\int_{V} \eta(\bar{x}, E) e^{-i \vec{k} \cdot g} d^{3} x+\rho_{0}(E) \int_{V} e^{-i k \cdot x} d^{3} x\right|^{2}
$$

Since the second integral in Eq. 3.1.4 is the contribution from forward scattering and is essentially a delta function centered at the origin and not experimentally distinguishable from the transmitted beam, it is common practice to ignore it. The equation for the structure factor is thus:

$$
S(\vec{k}, E)=\frac{1}{V}\left|\int_{V} \eta(\bar{x}, E) e^{-i \vec{k} \cdot \varepsilon} d^{3} x\right|^{2}
$$

Any variations from $\rho_{0}(E)$ due to voids and/or chemical inhomogeneity give rise to $\mathrm{x}$-ray scattering. The Babinet principle of reciprocity assures one that a deficiency in effective electron density (from the average) will lead to scattering of the same magnitude as that from an abundance of the same amount.

An equivalent description of the structure factor is to observe that it is the Fourier transform of the effective electron density pair correlation function. Explicitly writing out 
Eq. 3.1.5 and assuming the illuminated volume is large compared to the wavelengths of the Fourier components of interest:

$$
S(\bar{k}, E)=\frac{1}{V} \int_{V} e^{-i \vec{k} \cdot x} d^{3} x \int_{V} \eta(\bar{y}, E) \eta(\bar{x}+\bar{y}, E) d^{3} y .
$$

If the pair correlation function $\gamma(\bar{x}, E)$ is defined as the average over all two-point correlations of the change in effective electron density from the average, i.e.

$$
\gamma(\bar{x}, E)=\langle\eta(\vec{y}, E) \eta(\vec{x}+\bar{y}, E)\rangle=\frac{1}{V} \int_{V} \eta(\vec{y}, E) \eta(\vec{x}+\vec{y}, E) d^{3} y,
$$

then $\gamma(\vec{x}, E)$ and $S(\bar{k}, E)$ are Fourier transform pairs. That is,

$$
\gamma(\bar{x}, E)=\frac{1}{(2 \pi)^{3}} \int_{V} S(\bar{k}, E) e^{i k \cdot 8} d^{3} k
$$

It should be noted that the term "Fourier transform" is being loosely applied in these equations. Technically, the Fourier transform extends to infinity, so only if the electron density includes the change in density near and around the perimeter of the sample can the volume integral be extended to infinity. Realistically, however, the size of the illuminated sample is so large that the additional Fourier components introduced by the finite size effect are not important in this analysis; the additional Fourier components are not only far from the size region of interest but also are not instrumentally resolvable.

One general parameter that follows directly from the correlation function and is independent of any model structure is the mean square fluctuation in effective electron density, $\left\langle\eta^{2}(E)\right\rangle$. This is readily obtained from the pair correlation function for $\bar{x}$ 
identically 0 (i.e. $\left.\gamma(\bar{x}-0, E)-\left\langle\eta^{2}(E)\right\rangle\right)$ and is commonly known as the total elastic crosssection:

$$
\left\langle\eta^{2}(E)\right\rangle=\frac{1}{(2 \pi)^{3}} \int S(\bar{k}, E) d^{3} k
$$

It should be noted that no assumptions have been made about the sample at this point. Poorly defined phase boundaries, anisotropies, etc. may be present on any scale.

\subsection{Differential Anomalous Scattering}

Species-specific structural information manifests itself in the structure factor, which, for a binary alloy, consists of three specific contributions, $S_{\alpha \beta}(\vec{k})$, known as the partial structure factors (PSFs). Inserting Eq. 3.1.2 into Eq. 3.1.5, we have

$$
\begin{aligned}
S(\vec{k}, E)= & x_{A}\left|f_{A}(E)\right|^{2} S_{A A}(\vec{k})+2 \sqrt{x_{A} x_{B}} \operatorname{Re}\left[f_{A}(E) f_{B}^{*}(E)\right] S_{A B}(\vec{k}) \\
& +x_{B}\left|f_{B}(E)\right|^{2} S_{B B}(\vec{k})
\end{aligned}
$$

with $x_{A}$ the $A$ atomic fraction $\left(x_{B}=1-x_{A}\right)$,

$$
S_{\alpha \beta}(\bar{k})=\frac{1}{\sqrt{N_{\alpha} N_{\beta}}} \tilde{n}_{\alpha}(\vec{k}) \tilde{n}_{\beta}^{\cdot}(\bar{k})
$$

$\tilde{n}_{\alpha}(\bar{k})$ the Fourier transform of the difference in atom $\alpha$ 's number density from the average, and $N_{a}$ the number of $\alpha$ atoms in volume $V$. The derivation of Eq. 3.2.1 assumes that there exists a center of symmetry in the pair correlation function (i.e. $S_{A B}$ is real), which is always true for isotropic materials and assumed true even for this discussion of anisotropic scattering. This need not be the case, however, but it is a subtle effect and difficult to 
measure in amorphous materials. The DAS method ${ }^{2}$ requires at least three independent data sets near atomic absorption edges where the scattering factors are changing rapidly and hence modifying the PSF weighting functions. By observing the effect of various combinations of weighting functions on $S(\vec{k}, E)$, the PSFs can be extracted.

This is not trivial. The scattering factors that comprise the weighting functions differ with energy, but usually at most only $12-15 \%$ of the total scattering factor (for the $\mathrm{Mo}, \mathrm{Ge}$, and Fe K-edges studied in this work), so that the solution of Eq. 3.2.1 is illconditioned. Additionally, if the spectra exhibit only subtle variations with energy, good SNRs are required to produce statistically significant changes with energy. There have been several different approaches that have been tried over the years to obtain the PSFs, using linear and non-linear methods.

\section{2.a Weighted Average PSFs}

A simple yet general approach to determine the weighted average of a particular atom's PSF has been suggested by Shevchik. ${ }^{3}$ In this scheme, the derivative of the total structure factor with respect to energy is calculated for energies just below the atom's absorption edge, where the real part of the atom's ASF changes while the imaginary part and other atom's ASFs do not. By taking the derivative with respect to the varying $f_{A}^{\prime}$ of an A-B binary alloy and neglecting the small changes of $f_{A}^{\prime \prime}$ with energy, the equation is simpler:

$$
\partial S(\vec{k}, E) / \partial f_{A}^{\prime}=2 x_{A}\left|f_{A}(E)\right| S_{A A}(\vec{k})+2 \sqrt{x_{A} x_{B}}\left|f_{B}(E)\right| S_{A B}(\vec{k}) .
$$

Rice et al. ${ }^{4}$ used this approach to assess the degree of homogeneity of a sample. If there is no change in $S(\vec{k}, E)$ with $f_{A}^{\prime}(E)$, i.e. 


$$
0=\sqrt{x_{A}}\left|f_{A}(E)\right| S_{A A}(\bar{k})+\sqrt{x_{B}}\left|f_{B}(E)\right| S_{A B}(\bar{k})
$$

then $S_{A}(\vec{k} \neq 0)=0$ since $f_{A}(E)$ changes while $f_{B}(E)$ is constant. This consequently also leads to $S_{A B}(\vec{k} \approx 0)=0$; from Eq. 3.2.2, then, atom A must be distributed homogeneously $\left(\tilde{n}_{A}(\vec{k} \neq 0)=0\right)$. This result is also observed even without taking the derivative. If there is absolutely no change at the A edge (i.e. $S(\vec{k}, E)$ constant for various $f_{A}^{\prime}(E)$ ), then Eq. 3.2.1 implies $S_{A B}=S_{A A}=0$ for $k \neq 0$. The number density of atom A must be constant throughout the sample and thus a relative criterion for determining the degree of atom A's homogeneity in a sample has been established. In such a scenario, it is important to verify that atom B's PSF fully account for the changes in $S(\vec{k}, E)$, or else the equality in Eq. 3.2 .1 is violated.

\section{2.b Keating and Munro Approaches}

The Keating approach is the most straightforward method used to extract the PSFs and relies on writing Eq. 3.2.1 as a system of linear equations,

$$
W \cdot S_{P S F}(\bar{k})=S(\bar{k}, E) .
$$

For three independent measurements, the matrices are defined as:

$$
S(\vec{k}, E)=\left[\begin{array}{l}
S\left(\vec{k}, E_{1}\right) \\
S\left(\vec{k}, E_{2}\right) \\
S\left(\vec{k}, E_{3}\right)
\end{array}\right], W=\left[\begin{array}{lll}
x_{a}\left|f_{a}\left(E_{1}\right)\right|^{2} & 2 x_{b} \operatorname{Re}\left[f_{b}\left(E_{1}\right) f_{a}^{*}\left(E_{1}\right)\right\} & x_{b}\left|f_{b}\left(E_{1}\right)\right|^{2} \\
x_{a}\left|f_{a}\left(E_{2}\right)\right|^{2} & 2 x_{b} \operatorname{Re}\left[f_{b}\left(E_{2}\right) f_{a}^{*}\left(E_{2}\right)\right\} & x_{b}\left|f_{b}\left(E_{2}\right)\right|^{2} \\
x_{a}\left|f_{a}\left(E_{3}\right)\right|^{2} & 2 x_{b} \operatorname{Re}\left[f_{b}\left(E_{3}\right) f_{a}^{*}\left(E_{3}\right)\right. & x_{b}\left|f_{b}\left(E_{3}\right)\right|^{2}
\end{array}\right],
$$


and similarly for $S_{\mathrm{PSF}}(\vec{k})$. Eq. 3.2 .5 is usually solved by matrix inversion methods. The basic problem, however, is that the rows of the $3 \times 3$ weighting matrix $W$ are not sufficiently linearly independent to obtain a reliable matrix inversion: the $3 \times 3$ weighting matrix has such a large condition number that any systematic errors in the data and/or weighting matrix (e.g. scattering factors or atomic fraction) or poor SNRs become magnified and overwhelm the species-specific contributions. Modern methods have attempted to improve the condition number of the weighting matrix. Munro incorporates difference equations in the system of equations, and, with such a scheme, a maximum of 5 energies can be used:

$$
W^{\prime} \cdot S_{P S F}(\bar{k})=S^{\prime}(\bar{k}, E)
$$

with

$$
\begin{gathered}
S^{\prime}(\vec{k}, E)=\left[\begin{array}{c}
\Delta_{12}[S(\vec{k}, E)] \\
S\left(\vec{k}, E_{3}\right) \\
\Delta_{45}[S(\vec{k}, E)]
\end{array}\right], \\
W^{\prime}=\left[\begin{array}{ccc}
x_{a} \Delta_{12}\left[\left|f_{a}(E)\right|^{2}\right] & 2 x_{b} \Delta_{12}\left[\operatorname{Re}\left[f_{b}(E) f_{a}^{*}(E)\right]\right] & 0 \\
x_{a}\left|f_{a}\left(E_{3}\right)\right|^{2} & 2 x_{b} \operatorname{Re}\left[f_{b}\left(E_{3}\right) f_{a}^{*}\left(E_{3}\right)\right] & x_{b}\left|f_{b}\left(E_{3}\right)\right|^{2} \\
0 & 2 x_{b} \Delta_{45}\left[\operatorname{Re}\left[f_{b}\left(E_{3}\right) f_{a}^{*}\left(E_{3}\right)\right]\right] & x_{b} \Delta_{45}\left[\left|f_{b}\left(E_{3}\right)\right|^{2}\right]
\end{array}\right],
\end{gathered}
$$

and $\Delta_{i j}$ the difference of the quantity in brackets measured at energies $i$ and $j$. This improves the condition number and allows for the subtraction of instrumental errors that are present in scans 1 and 2 (or 4 and 5) but not in their difference. Since matrix methods can also be used to solve $m$ equations with $n$ unknowns, the Keating and Munro $3 \times 3$ weighting matrices can be expanded to an $m \times 3$ matrix, with $m$ the number of scans recorded. It is not 
clear that this is any better than using the 3 or 5 best scans at different energies, however, since the $m \times 3$ matrix will be even less linearly independent than the original $3 \times 3$ matrix.

\subsection{X-Ray Optics}

In order to perform anomalous scattering experiments, special care must be taken to ensure that there is adequate flux and energy resolution. Beamline 4-2 at the Stanford Synchrotron Radiation Laboratory, which is an 8-pole wiggler end-station, is the site of the semi-permanent ASAXS camera (see figure 3.2). The basic line consists of a Pt-coated toroidal mirror and a double-crystal monochromator. For $\mathrm{x}$-ray energies near the $\mathrm{Fe}$ and Ge K-edges, the $\mathrm{Si}(11 i)$ crystals and the mirror were used; near the Mo K-edge, an unfocused beam with $\mathrm{Si}(220)$ crystals were employed. The Si(111) crystals are better at the lower energies, since its second harmonic (222) is forbidden and its larger Darwin width allows greater intensity at no loss of energy resolution (see below). The Pt L-edges (just higher in energy than the Ge K-edge) help limit the higher order harmonics for $\mathrm{X}$-ray energies near the $\mathrm{Fe}$ and $\mathrm{Ge} \mathrm{K}$-edges, but the entire mirror must be removed for experiments at the Mo K-edge. Even with the mirror in place, the Si(111) crystals are still detuned $\sim 20 \%$. Near the Mo K-edge, however, the $\mathrm{Si}(111)$ crystals cannot be used due to small Bragg angles which lead to most of the beam passing over the top (or bottom) of the first crystal and are replaced with the $\operatorname{Si}(220)$ crystals (detuned $~ 50 \%$ ).

The energy resolution is determined by the Darwin width of the crystals and/or the beam divergence. The Darwin width for a first order reflection from planes parallel to the cleavage face $(h k l)$ for $\mathrm{x}$-rays polarized normal to the scattering plane is 5

$$
\Delta \theta=2.12 r_{e} \frac{n_{s i} \lambda^{2}}{\pi \sin 2 \theta}\left|F_{h k l}\right|
$$


(a) Scattering Geometry
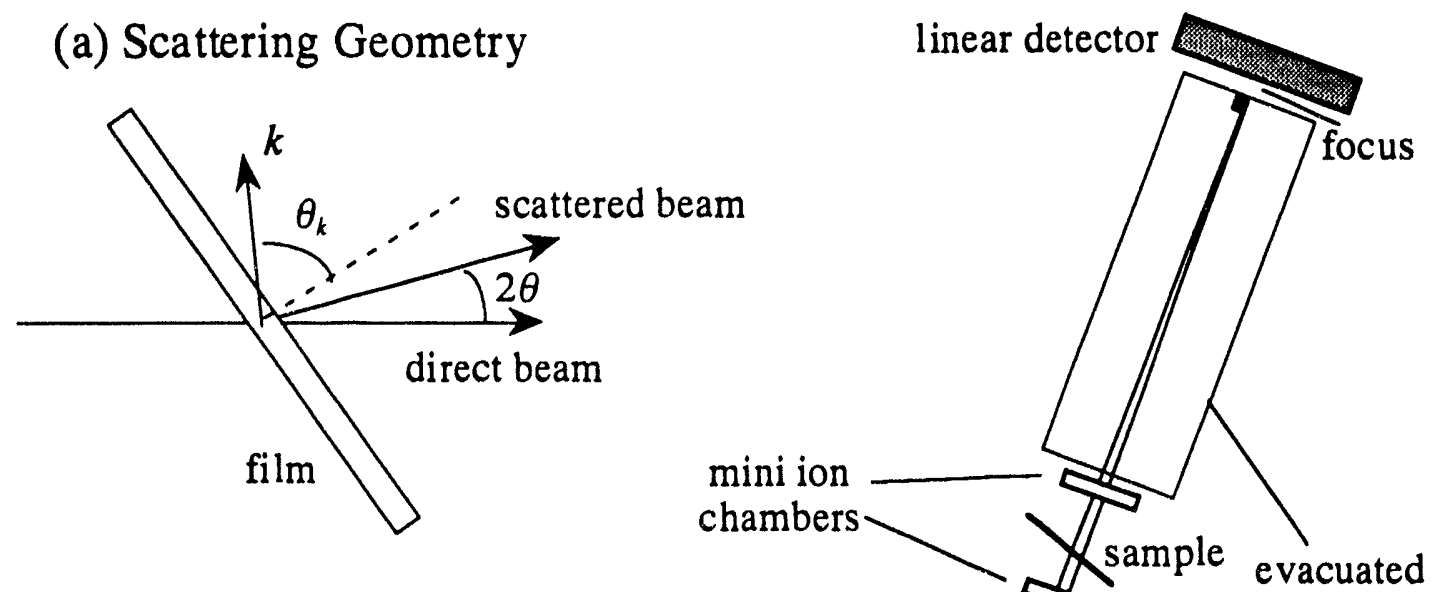

(b) 4-2 X-Ray Optics and SAXS Camera

4-2 hutch wall chambers

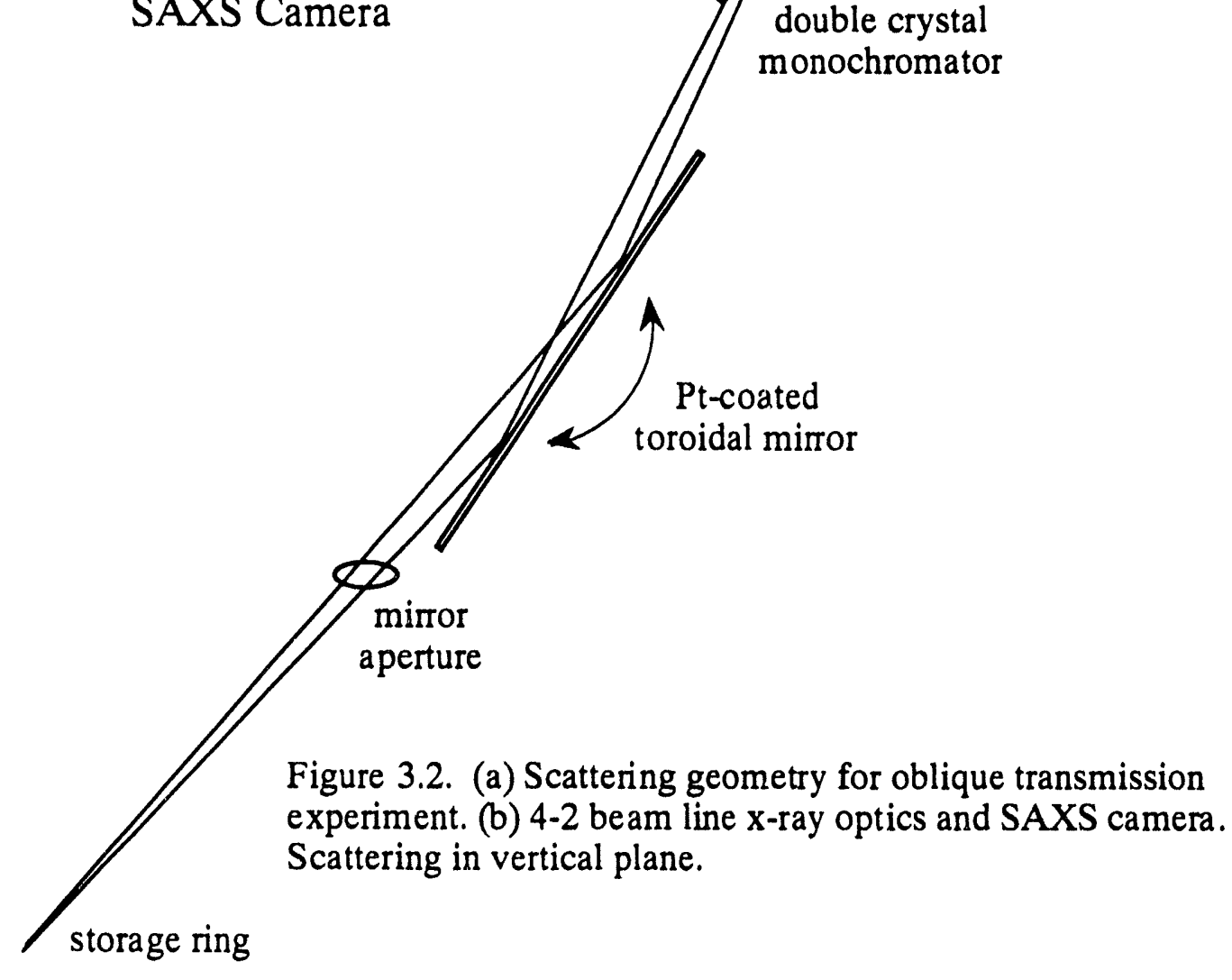


with $n_{S i}=0.0498 \AA^{-3}$ the number density of $\mathrm{Si}$ and $F_{h k l}$ the scattering amplitude of the $h k l$ reflection per $\mathrm{Si}$ atom $\left(F_{111}=5.657\right.$ and $F_{220}=8$.). The FWHM energy bandpass is then readily determined from the Bragg Law, $\Delta E / E=-\Delta \theta \cot \theta$. Table 3.1 shows the Darwin width and the associated bandpass of each crystal near the energies of interest. The bandpass is no greater than $1.2 \mathrm{eV}$ for all energies and crystals in table 3.1. This places an upper limit on the total intensity but is unfortunately not always the true energy resolution. The beam divergence and any focusing optics that are included (e.g. mirror at the Fe and Ge K-edges) must also be considered.

Table 3.1. Darwin widths and associated energy resolution for Si mono crystals.

\begin{tabular}{|l|l|l|l|l|l|l|}
\hline \multirow{2}{*}{$\begin{array}{l}\text { Energy } \\
(\mathrm{eV})\end{array}$} & \multicolumn{3}{|c|}{$\mathrm{Si}(111)$} & \multicolumn{3}{c|}{$\mathrm{Si}(220)$} \\
\cline { 2 - 7 } & $\theta$ & $\Delta \theta(\mu \mathrm{rad})$ & $\Delta E(\mathrm{eV})$ & $\theta\left(^{\circ}\right)$ & $\Delta \theta(\mu \mathrm{rad})$ & $\Delta E(\mathrm{eV})$ \\
\hline 7100 & 16.18 & 30.6 & 0.75 & 27.07 & 28.6 & 0.40 \\
\hline 11100 & 10.27 & 19.1 & 1.17 & 16.92 & 17.0 & 0.62 \\
\hline 20000 & N/A & N/A & N/A & 9.296 & 9.16 & 1.12 \\
\hline
\end{tabular}

For the case of point-to-point focusing with a mirror present, where the finite spot size is ignored, the energy resolution can be determined by considering the opening $\left(\gamma_{1}\right)$ and closing $\left(\gamma_{2}\right)$ angles and the distance of the mirror between the source $\left(l_{1}\right)$ and focus $\left(l_{2}\right)$, see figure 3.3. The largest angular difference between two rays that meet the Bragg relationship on the second crystal is given by

$$
\Delta \theta \equiv \theta_{2}-\theta_{1}=\gamma_{2}
$$

The focusing element determines the relationship between the opening and closing angles, which, for small angles, is $l_{1} \gamma_{1}=l_{2} \gamma_{2}$, or $\gamma_{1} \sim \gamma_{2}$ for the $1: 1$ focusing mirror on line 4-2. The 
horizontal) separated by an evacuated chamber roughly one to three meters in length. The samples are held in transmission and separated by a telescoping, evacuated chamber from a linear position-sensitive detector. The lead beamstop is glued in the middle of a thin mylar sheet that is rigidly attached to an aluminum ring ( $-25 \mathrm{~cm}$ diameter); the ring is placed inside the second of two evacuated chambers, centered on the direct beam, and motor controlled. Miniature ionization chambers, placed directly before and after the sample, are used to normalize scattering data as well as calibrate the monochromator. The monochromator, ion chambers, and detector are all controlled by a VAXstation through a CAMAC interface. ${ }^{6}$

Depending on the $\mathrm{x}$-ray energy, the windows used to separate the evacuated chambers and air are either 0.001 " mica or kapton. Mica is the preferred material, except that it contains enough $\mathrm{Fe}$ to prohibit its use at the $\mathrm{Fe}$ edge. Consequently, mica has been used at the Mo and Ge-edges, with kapton the material of choice at the Fe edge (and occasionally used at the $\mathrm{Ge}$ edge as well). Unfortunately, kapton has appreciable smallangle scattering in the angular region of interest, and, even with the free-standing films, the kapton scattering from the windows on the evacuated chambers is noticeable and must be subtracted. Details of data subtraction and normalization are presented next. Especiaily at the Mo edge, where the reduced counting efficiency limits the SNR, elimination of all kapton with mica (June, 1993) led to significant changes in the scattering pattern with energy which were somewhat obscured during a previous attempt (February, 1993).

Experiments had been performed on films held perpendicular to the $x$-rays, so that the scattering vector lies in the plane of the sample. Consequently, only those correlations in electron density projected onto the sample surface plane contributed to the scattering. The oblique transmission experiments allow for various orientations of the scattering vector. By tilting the sample an amount $\theta_{k}$, the scattering vector rotates out of the surface plane, and correlations in electron density are projected onto the plane defined at various angles with respect to the surface plane. A Huber 410 circle provided the necessary sample 
rotation; the circle in turn was affixed to an $\mathrm{XY}$ sample positioner which fit on the camera's optical rail. The data sets consist of radial scans in $\vec{k}$-space; that is, the sample is taken to a particular tilt angle $\theta_{k}$ so in the small-angle limit the direction of $\vec{k}$ is fixed, and the scattering is measured as a function of the scattering vector magnitude, $k$. This is done for $\theta_{k}=90,75,60,45,30,15^{\circ}\left(0^{\circ}\right.$ is not measurable) and, with the anomalous scattering performed at each angle, results in a total of 50-70 scans for each sample.

The linear position sensitive detector used in the measurements is a $20 \mathrm{~cm}$ linear position sensitive proportional counter supplied by Biologic. The details of the detector have been outlined by previous users ${ }^{7}$ and will not be discussed in detail. One point, however, must be stressed. At any point along the length of the linear detector, the efficiency is proportional to the absorption of photons in the depth of the detector (which is $6 \mathrm{~mm}$ for this particular model), $1-e^{-\mu(E) T}$, with $\mu(E)$ the absorption cross-section of the counter gas and $T$ the detector conversion depth. If the detector is too deep (large $T$ ) and if the incoming photon enters the detector at any angle with respect to the wire normal, then the position of the incoming photon is necessarily obscured. On the other hand, if it is too shallow (small $T$ ), the count rate is limited. Near the Fe K-edge, for example $E=7012 \mathrm{eV}$, the absorption length is $\mu(7000)^{-1}=4.0 \mathrm{~cm}$ for a $70 \% \mathrm{Ar} /$ balance $\mathrm{CO}_{2}$ mixture. Hence, the Ar mixture is usable at this photon energy with the detector of conversion depth $6 \mathrm{~mm}$. Near the Mo K-edge, the absorption length is $\mu(19900)^{-1}=77 \mathrm{~cm}$ for the same Arcontaining gas. This of course makes it a poor choice of gas. A better choice is one with a greater absorption cross-section, for example $80 \% \mathrm{Xe} /$ balance $\mathrm{CO}_{2}$ (although far more expensive). With this gas, $\mu(19900)^{-1}$ is $8.2 \mathrm{~cm}$, which leads to much improved counting statistics than with the lighter gas. Figure 3.4 compares the absorption cross-sections of the two gas mixtures as a function of energy. Clearly, the Xe filled gas is the superior choice. At the Mo and $\mathrm{Ge} \mathrm{K}$-edges, the $\mathrm{Xe} / \mathrm{CO}_{2}$ gas has been used, while the detector has been filled with $\mathrm{Ar} / \mathrm{CO}_{2}$ at both the $\mathrm{Fe}$ and $\mathrm{Ge} \mathrm{K}$-edges. 
(a)

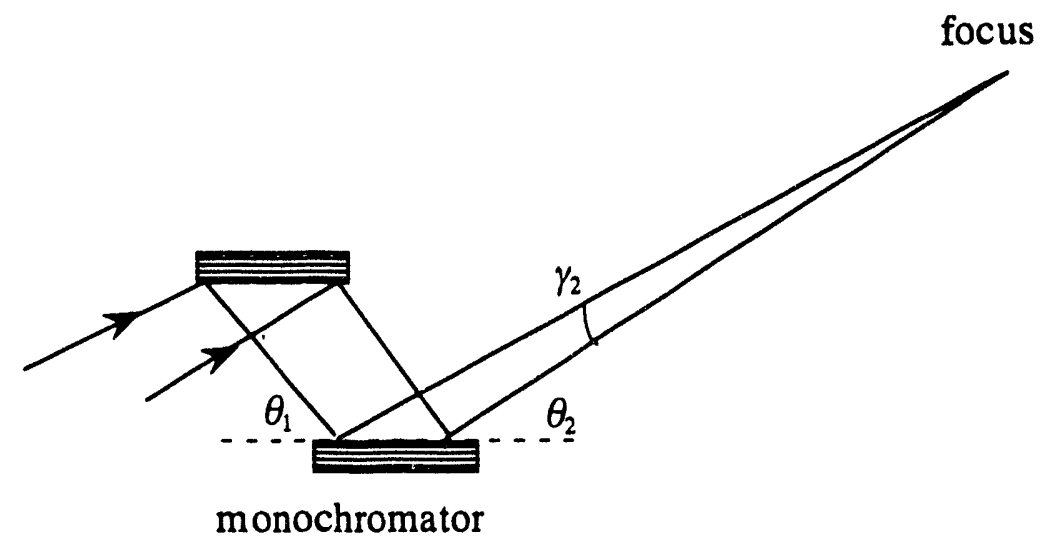

(b)

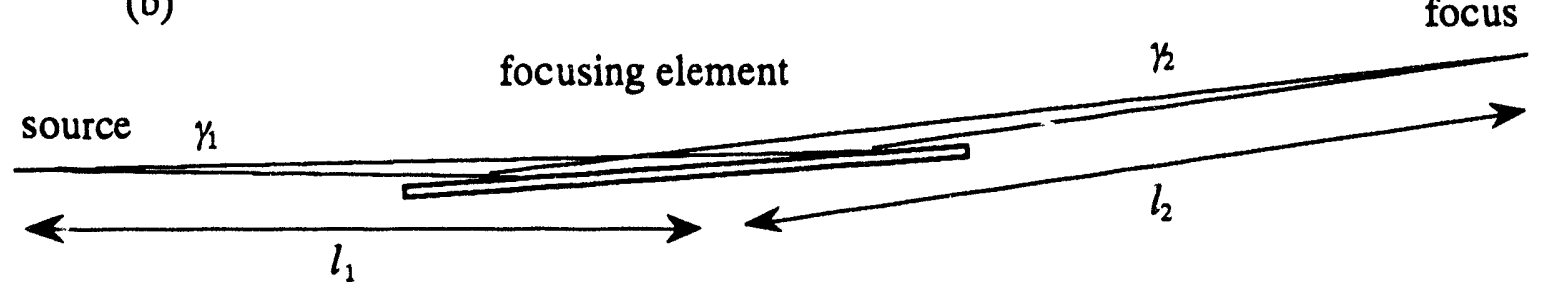

Figure 3.3. Energy resolution and the influence of focusing elements. $\gamma_{1}$ and $\gamma_{2}$ are the opening and closing angles, with $l_{1}$ and $l_{2}$ the distances between the source and mirror and mirror and focus, respectively. For beamline $4-2$ with a $1: 1$ focusing mirror, $l_{1} \sim l_{2}$ and thus $\gamma_{1} \sim \gamma_{2}$. 
vertical divergence has been kept to $\gamma_{1}=64.5 \mu \mathrm{rad}$ with the mirror aperture which thus provides $\Delta \theta=64.5 \mu \mathrm{rad}$ with an associated energy resolution of $\Delta E=1.58$ and $3.95 \mathrm{eV}$ for $\mathrm{Si}(111)$ crystals at $E=7100$ and $11100 \mathrm{eV}$, respectively. It should be noted that with such a small $\gamma_{1}$ (which has been intentionally chosen to provide such energy resolution), the mirror probably does not provide more than a factor of 4-5 increase of the total flux but is still used as a low-pass filter. Moreover, since the Darwin width is smaller than the source divergence, the narrow widths serve only to limit flux and do not serve to improve the energy resolution. It would, of course, be better to use crystals with a Darwin width that approached the vertical divergence of $64.5 \mu \mathrm{rad}$. At the Mo K-edge with the $\operatorname{Si}(220)$ crystals and the mirror withdrawn, the energy resolution is determined by the opening angle, $\gamma_{1}$, and is thus $\Delta E=7.88 \mathrm{eV}$ for the $\mathrm{Si}(220)$ crystals at $20 \mathrm{keV}$.

For the ASAXS experiments, then, the energy resolution is a combination of the Darwin bandpass and that due to beam divergence; for these measurements, the vertical divergence is the limiting factor. We typically work at 4-6 incident $\mathrm{x}$-ray energies below each edge. At the Fe K-edge: $-200,-100,-50,-20,-10 \mathrm{eV}$ (edge at $7112 \mathrm{eV}$ ); the Ge K edge $-300,-200,-100,-50,-20,-10 \mathrm{eV}$ (edge at $11103 \mathrm{eV}$ ); the Mo K-edge: $-400,-100$, $-20,-10 \mathrm{eV}$ (edge at $20000 \mathrm{eV}$ ). The energy bandpass is thus sufficiently narrow to observe and record the anomalous scattering effects.

\subsection{SSRL Small-Angle Scattering Camera}

The SAXS camera used for the measurements is outlined in figure 3.2. The mirror aperture and a set of slits before the monochromator are used to define the beam and limit its divergence as it travels towards the hutch. Once in the hutch, the incident beam is further defined by two sets of slits (each approximately $0.25 \mathrm{~mm}$ vertical by $0.50 \mathrm{~mm}$ 

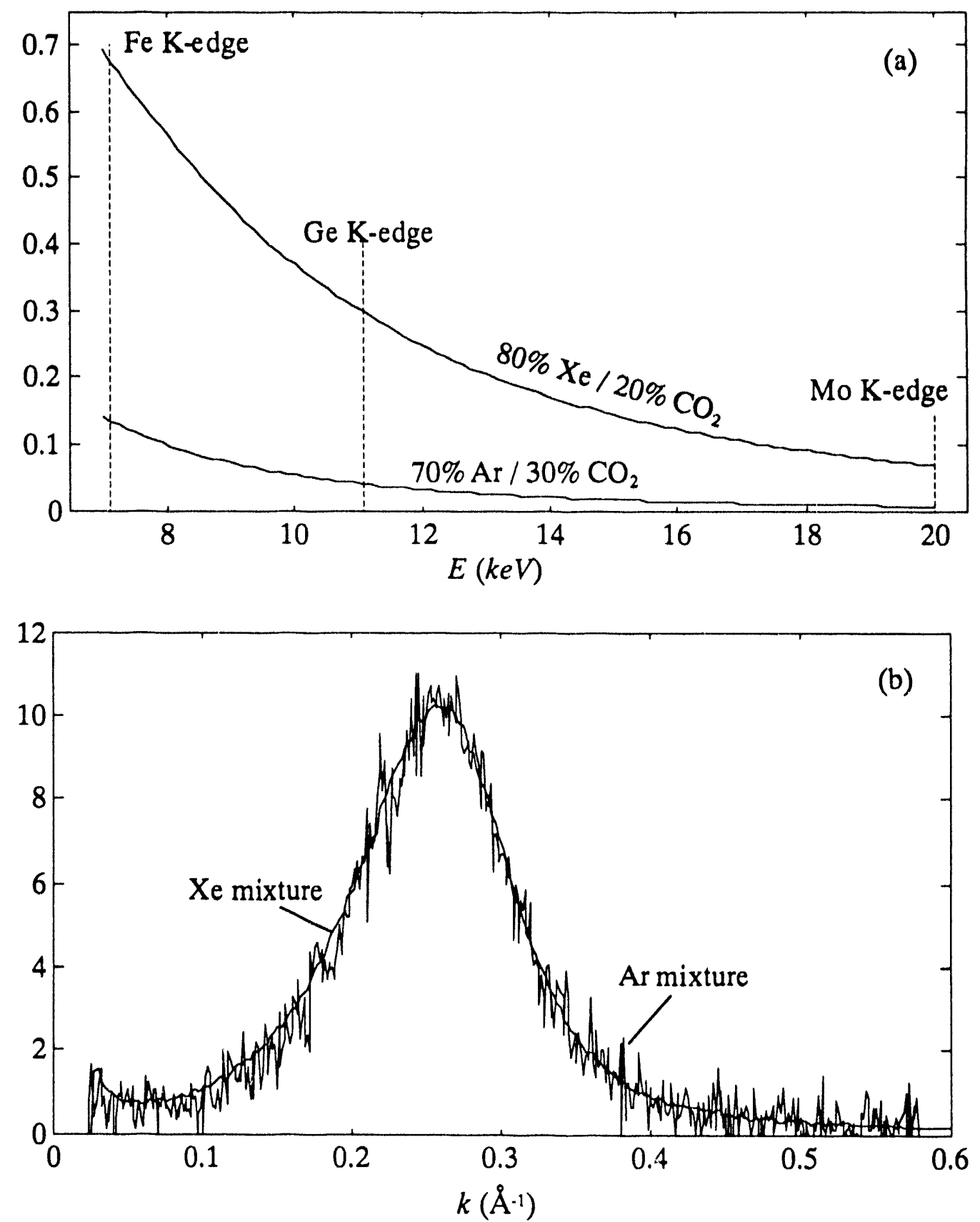

Figure 3.4. (a) Theoretical comparison of the efficiencies for gas mixtures of $\mathrm{Xe} / \mathrm{CO}_{2}$ and $\mathrm{Ar} / \mathrm{CO}_{2}$ with a $6 \mathrm{~mm}$ conversion depth as a function of photon energy (absorption from $\mathrm{Be}$ windows, air, etc. not included). (b) Absolute scaled scans at normal incidence for the 13.6 at.\% Fe sample with the Ar and Xe gas mixtures at $E=11093 \mathrm{eV}$. Similar data collection time for each scan with no smoothing performed. The Xe/CO gives a superior signal due to its greater absorption cross-section at the Ge and Mo-edges than Ar. ASAXS near the Mo K-edge is simply not possible with an $\mathrm{Ar} / \mathrm{CO}_{2}$ mixture. 
There is another problem, however. With an incident energy near the Mo K-edge, Ge fluorescence $\left(K \alpha_{1}=9886, K \alpha_{2}=9855, K \beta_{1}=10982, K \beta_{3}=10975 \mathrm{eV}\right)$ from the Mo-Ge samples swamp the detector, since the fluorescence is at lower energies and hence preferentially absorbed in the limited detector depth $\left(\mu(10500)^{-1}=1.5 \mathrm{~cm}\right.$ for the Xe mixture). With the Xe mixture and large fluorescence signal, the count rates are so large that space-charge effects and dead time corrections become appreciable. The energy resolution of the detector is approximately $20 \%$, so the fluorescence can be discriminated and excluded from the coherent signal. However, the beam flux must be reduced in any case to avoid the space-charge and deadtime effects, and the resulting count rate is not good enough to perform anomalous scattering. Another method is to employ absorbing filters with an absorption edge between the fluorescent radiation and the coherent signal (e.g. $\mathrm{Zn}$ with a K-edge at $\sim 9700 \mathrm{eV}$ ). However, placing such filters after the sample, for example, leads to fluorescence from the filter itself. This problem has been overcome by sputtering $-4.8 \mu \mathrm{m} \mathrm{Zn}$ onto a large sheet of $0.001 "$ kapton. The $\mathrm{Zn}$ filter is then placed over the detector, downstream of the beamstop, so the Ge fluorescence can be preferentially absorbed without excitation of $\mathrm{Zn}$ fluorescence from the direct beam. Two filters, stacked one on top of the other for a net $-9.6 \mu \mathrm{m} \mathrm{Zn}$, worked best. Of course not all $\mathrm{Ge}$ fluorescence is captured--Section 3.6 explains background subtraction. Fluorescence from $\mathrm{Fe}$ in the Fe-Ge samples was not so great as to warrant the use of an absorbing filter and was removed with the background subtraction method outlined later. This is most likely due to the small $\mathrm{Fe}$ concentrations studied and the low fluorescence yield expected from lighter elements.

\subsection{Data Normalization and the Absolute Scale}

Before the data collection and reduction procedure are discussed, it is instructive first to understand what is required to normalize and place the data on an absolute scale. 
Simply put, the absolute scaled differential cross-section is the number of photons that scatter into a given solid angle for a given time per incident flux on the sample. Since the observed SAXS is very weak, background scattering and sample absorption must be carefully considered. We suppose that an ion chamber $\left(I_{1}\right)$ is placed directly after the sample and that a linear detector is placed further downstream some distance from the sample with a number of absorbing elements (e.g. kapton, Be, or mica windows; air) in the path of the beam. For a given photon energy $E$ and incident intensity, window materials, and sample, the total scattering observed at any position $u$ along the length of the linear detector, $I_{\text {Tor }}(u)$, consists of two contributions, that from the sample, $I_{s}$, and all other sources, $I_{\text {other }}$. The window materials and the parasitic scatter around the main beam contribute to $I_{\text {other }}$. Since the only inelastic scattering at small angles is expected to result from the sample (fluorescence and resonant Raman scatter with only a negligible Compton shift at small angles), $I_{s}$ must be summed over all relevant photon energies:

$$
I_{\text {TOT }}(u)=\sum_{\dot{E} s E} I_{s}(u, \tilde{E})+I_{\text {other }}(u, E) .
$$

All units of intensity, $I$, are in counts/time and have not been corrected for the efficiency of the detecting system in order to give the absolute number of photons/sec. To understand how to remove the $I_{\text {other }}$, we must first consider the absorption of the sample.

For scattering at small angles with an oblique transmission geometry, the absorption correction is easy to calculate. For a given volume element $d V$ at a depth $z^{\prime}$ in the film, there are two absorption factors to consider. The first is the attenuation of the incident beam as it travels to depth $z^{\prime}$ in the sample, given by $e^{-\mu_{s}(E) z^{\prime}}$ with $\mu_{s}(E)$ the linear absorption coefficient for the sample and $t$ the film thickness. The second is the attenuation of the scattered beam as it traverses the remaining film, given by $e^{-\mu(\dot{E})\left(t / \sin \theta_{1}-z^{\prime}\right)}$ in the 
small-angle limit. The overall absorption correction factor for elastically scattered photons is the product of these factors, or $e^{-\mu,(E) / / \sin \theta_{t}}$.

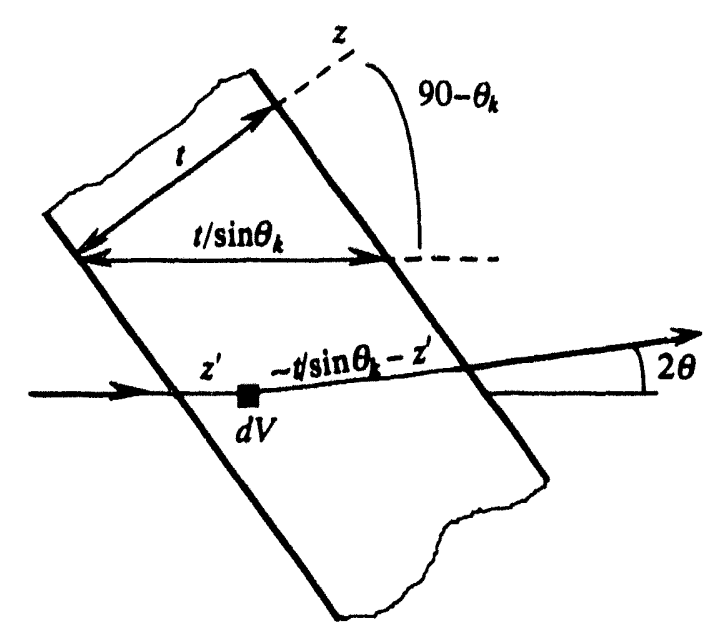

Figure 3.5. Absorption correction illustration for samples held in transmission.

With the sample in the path of the beam, the elastic scattering from the window materials is attenuated by $e^{-\mu_{,}(E) / s \sin \theta_{k}}$ and is removed by measuring the scattering with and without sample, $I_{\text {Tor }}(u)$ and $I_{\text {other }}(u, E)$ respectively, and then subtracting the two after removing the attenuation from the sample:

$$
\frac{\sum_{E_{\varepsilon} E} I_{s}(u, \tilde{E})}{I_{0} e^{-\mu_{t}(E) / \sin \theta_{t}}}=\frac{I_{\text {TOT }}(u)}{I_{0} e^{-\mu_{t}(E) / / \sin \theta_{t}}}-\frac{I_{\text {other }}^{\prime}(u, E)}{I_{0}^{\prime}}
$$

where an extra factor of $I_{0}$ (incident beam intensity) is included to account for changes in beam current and the prime indicates that the sample is removed. It is usually more practical to place an ion chamber immediately after the sample, to record $I_{1}=I_{0} e^{-\mu_{s}(E) / \sin \theta_{8}}$, in order to automatically include the sample absorption correction. With the sample removed, $I_{1}(E)$ is identical to $I_{0}$. Hence, Eq. (3.5.2) can be replaced with: 


$$
\frac{\sum_{E_{\varepsilon} \varepsilon E} I_{s}(u, \tilde{E})}{I_{1}(E)}=\frac{I_{T O T}(u)}{I_{1}(E)}-\frac{I_{\text {other }}^{\prime}(u, E)}{I_{1}^{\prime}(E)},
$$

The distance along the wire, $u$, is proportional to the magnitude of the momentum transfer $k$, which is determined by the distance from the sample to the detector and number of detector channels/length.

Now that it is clear how to isolate the sample scattering, we will focus on the differential cross-section of scattered radiation from the sample and the absolute scale. The scattering of a photon with final energy $\bar{E}$ from a differential volume element $d V$ in the sample given an incident energy $E$ can be written as

$$
\frac{\partial^{2}}{\partial V \partial \tilde{E}} I_{s}(\vec{k}, \tilde{E})=\kappa(E, \tilde{E}) I_{0} e^{-\mu_{s}(E) z} e^{-\mu_{s}(\tilde{E})\left(t / \sin \theta_{\varepsilon}-z\right)} \frac{d \sigma}{d \Omega_{V}}(\vec{k}, E, \tilde{E}),
$$

with the result scaled by the incident intensity, sample absorption, and the differential cross-section per unit volume, $d \sigma / d \Omega_{V} \cdot d \sigma / d \Omega_{V} \cdot d V$ is the number of scattered photons of energy $\tilde{E}$ from volume $d V$ into a solid angle $d \Omega$ given an incident flux of photons of energy $E$. The proportionality constant, $\kappa$, which defines the absolute scale, is the ratio of the efficiency of the $I_{0}$ (or $I_{1}$ ) ion chamber for energy $E$ to that of the linear detector for energy $\bar{E}$ multiplied by any extra absorption from the camera (e.g. detector's Be window, kapton or mica windows). $\kappa$ is also scaled by the effective solid angle $d \Omega$ spanned by each channel on the linear detector (which is the same for all channels along $u$ in the small-angle limit), in order to convert the $d \sigma / d \Omega_{V}$ on the right-side to intensity units on the left-side (Eq. 3.5.4).

As mentioned in Section 3.1, the inelastic scattering (fluorescence and resonant Raman scatter) is angle-independent but of course energy-dependent. Hence, we write the 
total differential cross-section per unit volume as the sum of the elastic and inelastic contributions:

$$
\frac{d \sigma}{d \Omega_{V}}(\vec{k}, E, \tilde{E})=\frac{d \sigma_{\text {elas }}}{d \Omega_{v}}(\vec{k}, E) \delta(E-\tilde{E})+\frac{d \sigma_{\text {inel }}}{d \Omega_{V}}(\tilde{E}<E)
$$

When substituted into Eq. 3.5.4 and integrated over $V$ and $\tilde{E}$, we have:

$$
\frac{d \sigma_{\text {elas }}}{d \Omega_{V}}(\vec{k}, E)+\Re(E)=\frac{\sum_{\dot{E} s E} I_{s}(\vec{k}, \tilde{E})}{I_{1}(E) t / \sin \theta_{k}} \frac{1}{\kappa(E) A}
$$

$A$ is the area of the beam defined by the slits, and $\mathfrak{R}(E)$ is given by

$$
\mathfrak{R}(E)=\int_{\dot{E}<E} d \tilde{E} \frac{\kappa(E, \tilde{E})}{\kappa(E) t / \sin \theta_{k}}\left[\frac{1-e^{-\left(\mu_{s}(\dot{E})-\mu_{s}(E)\right)_{t / s \sin \theta_{k}}}}{\mu_{s}(\tilde{E})-\mu_{s}(E)}\right] \cdot \frac{d \sigma_{\text {inel }}}{d \Omega_{v}}(E, \tilde{E})
$$

The $k$-independent function $\mathfrak{R}(E)$ is negligible for energies far from any absorption edges in the sample.

The elastic scattering from the sample will thus be superimposed on an energydependent 'background' determined by $\mathfrak{A}(E)$. Estimates of $d \sigma_{\text {inel }} / d \Omega_{v}$ can be found in the literature (e.g. for resonant Raman scatter, see Ref. 1), but without $\kappa(E, \tilde{E})$ and $\mu_{s}(\tilde{E})$ the overall function $\Re(E)$ cannot be determined. With the proper energy resolution, it would be possible to discriminate the elastic signal from $\mathfrak{R}(E)$. For the case of resonant Raman scattering of $\mathrm{Fe}$ at $E=7000 \mathrm{eV}$ (Fe K-edge is at $7112 \mathrm{eV}$ ), $\tilde{E}=6292$ (final state hole at LIII), 6279 (LII), and 6154 (LI) eV. With an energy resolution of only 20\%, the position sensitive proportional counter unfortunately cannot discriminate the resonant Raman from the elastic signal. The same is true at both the Ge and Mo K-edges. With 
more advanced detectors, it should be possible to exclude the resonant Raman signal. Methods are developed in Section 3.6 to remove the $\mathfrak{R}(E)$ and hence extract the elastic signal.

If the incident energy is below the $\mathrm{Fe} \mathrm{K}$ edge for the $\mathrm{Fe}-\mathrm{Ge}$ alloys or $\mathrm{Ge} \mathrm{K}$ edge for the Mo-Ge alloys, then the fluorescence should be minimized, with $\mathfrak{R}(E)$ consisting only of the resonant Raman contribution. There is still fluorescence from the L edges, but the characteristic radiation produced is at such low energies that it is easily absorbed by the window materials, air, etc. With only small changes in the sample's absorption from photons of energy $E$ to $\bar{E},\left(\mu_{s}(\bar{E})-\mu_{s}(E)\right) t / \sin \theta_{k} \ll 1$, Eq. 3.5 .7 can be simplified:

$$
\mathfrak{R}(E)=\int_{\dot{E}<E} d \tilde{E} \frac{\kappa(E, \tilde{E})}{\kappa(E)} \cdot \frac{d \sigma_{\text {inel }}}{d \Omega_{V}}(E, \tilde{E}),
$$

where the integration is performed over the relevant energies (e.g. $6100-6300$ for Fe-Ge and $E=7000)$. We are still limited, however, by the unknown $\kappa(E, \tilde{E})$, and it will not be possible to remove $\mathfrak{R}(E)$ from theoretical calculations of $d \sigma_{\text {inel }} / d \Omega_{v}$.

To arrive at the overall sum $d \sigma_{\text {elas }} / d \Omega_{v}+\mathfrak{R}(E)$, the quantity $\kappa(E)$ in Eq. 3.5.6 must be measured. Foils experiments ${ }^{8}$ can be used, where, for a particular energy, foils of different thicknesses are placed between $I_{1}$ and the linear detector. By extrapolation to 0 foils, $\kappa(E)$ can be determined. There are problems with this, however, especially with proportional counters. ${ }^{9}$ Space-charge and deadtime effects permit only low count rates ( $<1000 \mathrm{cps}$ on any one position of the wire) so that extrapolation to 0 foils is difficult to determine accurately. In addition, higher energy harmonics from the monochromator crystals can more easily penetrate the foils than the first-order reflection and tend to pollute the extrapolation. The preferred method is to use a secondary standard that has already been calibrated by a variety of methods. 
The secondary standard used in this work is a piece of polyethylene (PE) normalized by T.P. Russell et al. ${ }^{10}$ using small-angle $x$-ray and neutron scattering data. $\mathrm{PE}$, a strong small-angle scatterer with a well-defined scattering maximum at $k \sim 0.037 \AA^{-1}$, is a partially crystalline polymer which consists of $\mathrm{C}$ and $\mathrm{H}$ atoms. Since the absorption edges of these elements are far below the Fe K-edge energy, the $\mathrm{C}$ and $\mathrm{H}$ anomalous scattering factors are negligible, and the absolute scaled differential cross-section determined by Russell et al. is valid at photon energies near the $\mathrm{Fe}, \mathrm{Ge}$, and Mo K-edges. As with the window materials, the PE's inelastic scattering is negligible at small angles. The differential cross-section per unit volume of the PE standard is $d \sigma_{P E}\left(k^{\prime}\right) / d \Omega_{V}=4.25$ $\mathrm{cm}^{-1}$ for $k^{\prime}$ the scattering maximum. With short camera lengths, the PE maximum is not always resolvable, so a point was chosen at larger $k, k^{\prime \prime}=0.063 \AA^{-1}$, where the PE absolute scattering has been determined from long camera length measurements, $d \sigma_{P E}\left(k^{\prime \prime}\right) / d \Omega_{V}=1.93 \mathrm{~cm}^{-1}$. For changes of -200 to $0 \mathrm{eV}$ at the $\mathrm{Fe},-300$ to $0 \mathrm{eV}$ at the $\mathrm{Ge}$, and -400 to $0 \mathrm{eV}$ at the Mo K-edges, no changes in the PE's normalized intensity, $I_{P E}(k, E) / I_{1}(E)$, were resolvable. Apparently, the ratio of the efficiency of $I_{1}(E)$ to that of the linear delector (with either Ar or Xe gas mixtures) is essentially a constant over these small changes in energy. It should be noted that the absolute scale is difficult to achieve precisely--we suspect that there is probably a $10 \%$ error in the scale reported here.

The $d \sigma_{\text {elas }} / d \Omega_{V}+\Re(E)$ for a given sample, then, is determined by measuring PE under identical experimental conditions as that for the sample, subtracting the scattering from other sources (Eq. 3.5.3), and forming the following ratio to cancel the $\kappa(E) A$ in Eq. 3.5.6:

$$
\frac{d \sigma_{e l a s}}{d \Omega_{V}}(\bar{k}, E)+\Re(E)=\frac{\sum_{\tilde{E}_{s E}} I_{s}(\bar{k}, \tilde{E})}{I_{1}(E) t / \sin \theta_{k}} \cdot \frac{t_{P E} I_{1, P E}(E)}{I_{P E}\left(k^{\prime}, E\right)} \cdot \frac{d \sigma_{P E}}{d \Omega_{V}}\left(k^{\prime}, E\right),
$$


where $t_{P E}$ is the thickness of the PE.

\subsection{Data Collection and the Measured Structure Factor}

Data collection, normalization, and $\Re(E)$ subtraction are discussed next. For a given sample and tilt angle $\theta_{k}$, SAXS spectra are recorded at 4-6 energies, with typical scans at each energy $\sim 1-10 \mathrm{~min}$ in length. These are recorded cyclically; that is, spectra recorded and saved at each energy and then again, usually 4-6 times. The end result is usually 4-40 min collection time per angle per tilt angle $\theta_{k}$. There is essentially no dark current with the beam off ( $1-5$ cps integrated along the length of the detector), so that it is ignored. The data are centered, calibrated to a $\vec{k}$-scale, divided by $I_{1}(E)$, and averaged over cycles. ${ }^{7}$ In order to prevent extra background, no windows were placed on the $I_{1}$ ion chamber, so that the counter gas was simply hutch air at the $\mathrm{Fe}$ and $\mathrm{Ge} \mathrm{K}$-edges. At the Mo K-edge, however, the extra absorption cross-section from Ar was needed to improve the $I_{1}$ count rate--mylar windows were placed on the chamber and Ar flowed. Typical count rates for the linear detector are $-2000-20000 \mathrm{cps}$ integrated along the wire.

Finally, scattering from sources other than the sample is subtracted (Eq. 3.5.3) and the elastic scattering placed on an absolute scale with the PE standard (Eq. 3.5.8), as prescribed in the previous section. The range of reciprocal space covered, of course, depends on the sample-detector distance and the incident $\mathrm{x}$-ray energy, but typical values ranged from $k=0.005$ to $0.60 \AA^{-1}$. Smearing effects due to finite beam size have been considered but are negligible due to the small spot size used and relatively large smallangles studied and hence are ignored in the analysis. Figure 3.6 shows SAXS from the 18.2 at.\% $\mathrm{Fe}$ and 6.5 at.\% Mo samples near the $\mathrm{Fe}$ and $\mathrm{Ge} \mathrm{K}$-edges along with the background from the mica and kapton windows. The scattering from the mica windows is essentially flat in the scattering region of interest. Thus mica is a better window material 
than kapton, which has a weak ret comparatively strong scattering maximum near $k \sim 0.38$ $\AA^{-1}$. The x-ray signal from the sample itself is weak, no more than 3-5 times stronger than the background from other sources in the beam.

Figure 3.6 also shows two "dead spots" that are in (a) but not (b). During one of the periods of data collection, the detector had been partially destroyed by other users in those two regions. The proper way to correct for this problem is to uniformly irradiate the detector with $\mathrm{x}$-rays and measure the detector response function. Then, we divide this function into the collected scans, which should, in principle, correct for any changes in efficiency along the length of the linear detector. The easiest way to obtain the detector response function is to use $\mathrm{x}$-rays just above an absorption edge, for example $7200 \mathrm{eV}$ (just above the $\mathrm{Fe} \mathrm{K}$-edge). With an $\mathrm{Fe}$ filter in place, $\mathrm{Fe}$ characteristic radiation is emitted isotropically, which provides a strong signal with which to measure the response function. In addition, by placing the detector at $2 \theta-90^{\circ}$ and in the plane of the storage ring, there will be no Bragg scattering from the Fe filter due to the polarization of the synchrotron radiation. The detector response did not vary by more than $5 \%$ along the length of the detector and 1-2\% from channel to channel, except for the dead spots shown in figure 3.6a. In that case, the detector response function only imperfectly corrected for the dead spots (the degradation is a function of time and changes). The scattering maximum was intentionally oversampled ( 100 channels per $\left.0.1 \AA^{-1}\right)$, so that the dead area could be isolated as much as possible and discarded. In figure $3.6 \mathrm{~b}$, the linear detector was exceptionally clean with no dead channels. In that case, the sampling rate was decreased ( $\sim 40$ channels per $\left.0.1 \AA^{-1}\right)$.

There remains the issue of isolating the sample's elastic scattering, which is superimposed on the angular-independent, inelastic scattering from fluorescence and the resonant Raman effect. Methods in the literature have focused on the scattering at large $k$. 

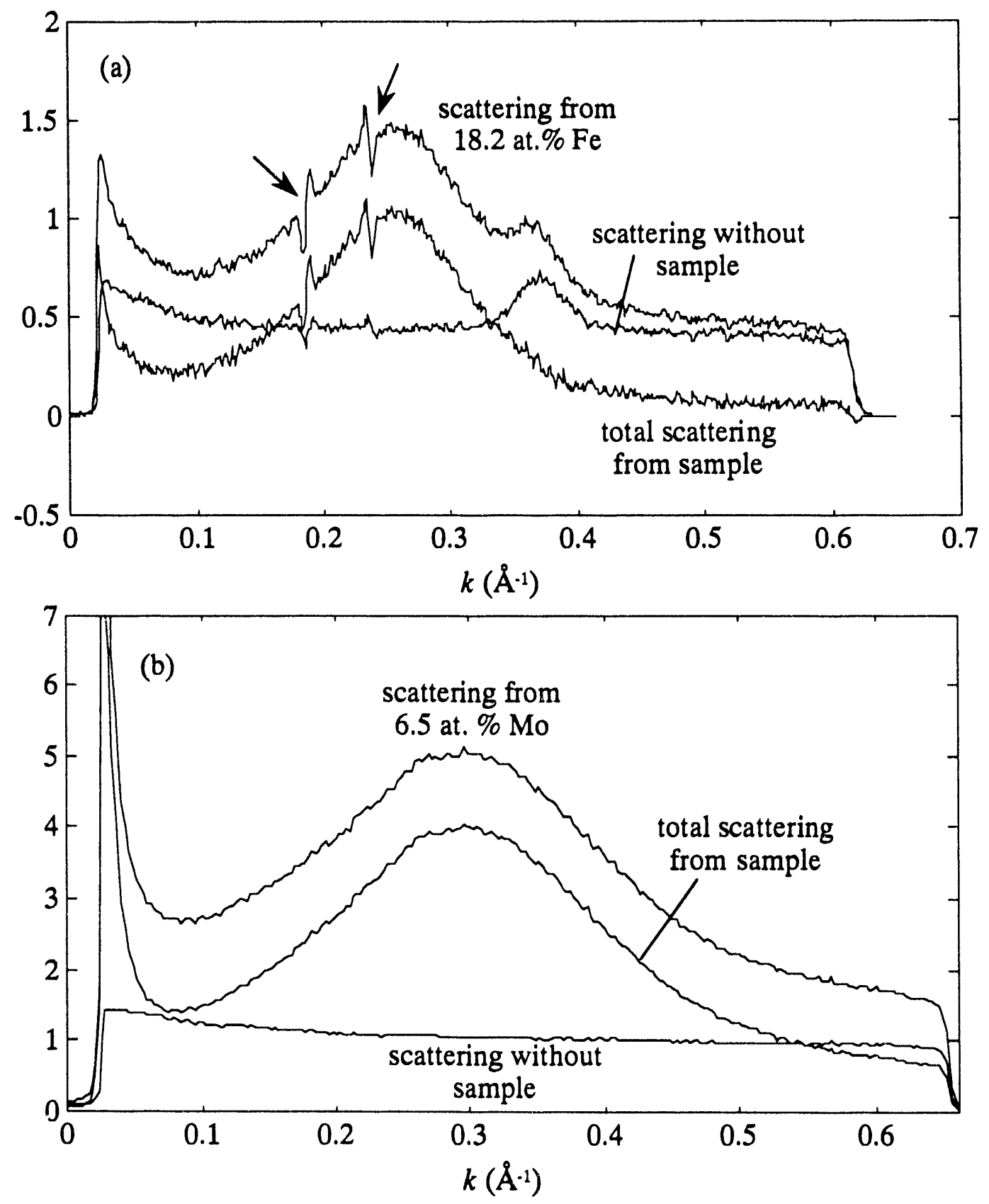

Figure 3.6. Scattering from the free-standing films: subtraction of scattering from window materials, prior to being placed on an absolute scale with the PE. The total recorded scattering is $I_{T O T}(\vec{k}) / I_{1}(E)$, with $I_{\text {TOT }}(\vec{k}) / I_{1}(E)-I_{\text {other }}^{\prime}(u, E) / I_{1}^{\prime}(E)$ the total scattering from the sample (see Eq. 3.5.3). In (a), recorded at $6912 \mathrm{eV}$ with an Ar counter gas, 0.001" kapton windows on the evacuated chambers contribute to the observed SAXS and are subtracted. The arrows show that there are two dead spots along the detector wire which must be corrected or smoothed (see text). (b) The 0.001 " mica windows have essentially a flat background in the scattering region of interest and are thus a better window material than kapton ( $E=10803 \mathrm{eV}$ with Xe gas mixture). 
For the case of the relatively strong-scattering $\mathrm{Cu}-\mathrm{Ni}-\mathrm{Fe}$ alloys, Simon and $\mathrm{Lyon}^{11}$ noticed little angle-dependent scattering for $k>0.35 \AA^{-1}$ and hence were able to remove the flat background by aligning all scans to 0 for $k>0.35 \AA^{-1}$. For the case of a broad SAXS maximum as observed in this work, Maret et al. ${ }^{12}$ fit the Porod law $\left(S(k) \propto 1 / k^{4}\right)$ and a constant background to the large $k$ scattering observed from a-TbCu and a-GdCu alloys. Due to the inverse relation between real and reciprocal space, at large $k$ the elastic scattering originates from the interface between distinct regions of different electron density (e.g. particle and matrix), often referred to as the surface of the particle. $k$ is still small compared to atomic dimensions. If the surface layer between the regions of electron density or particles and matrix is a sharp, abrupt density transition, then large $k$ scattering follows the Porod $k^{-4}$ law. Although they felt "the Porod asymptotic behavior is too inaccurate to give a reliable corrected value" for the structure factor, it is not clear if the problem of using such a method to correct for background is in the SNR of their data, the small $k$-range fit, or a fundamental problem with power-law scattering. In fact, Schmidt et al..$^{13}$ have found that power-law relationships $\left(S(k) \propto 1 / k^{\alpha}\right)$ that are fit over several decades in $k$ may differ $(-10 \%)$ from a local power-law fit over smaller ranges in $k$. The regions need not be of a particular size, identical in shape, or dilute in particle density.

Many years ago, Ruland ${ }^{14}$ showed that if the density transition is not sharp between the two phases, then one can approximate the transition from one phase to another as the convolution of a Gaussian with the step function. The Gaussian describes the interfacial width of the transition, and the step function, a perfect interface. With the Fourier deconvolution theorem, the observed scattering at large $k$ is thus:

$$
S(k) \propto \frac{\exp \left(-\sigma_{w}^{2} k^{2}\right)}{k^{4}},
$$


with the step function transforming as the Porod law and the Gaussian into another Gaussian. $\sigma_{w}$ is the standard deviation of the smoothing function, with the total interfacial width taken as $2 \sigma_{w}$. By plotting $\log \left[k^{4} S(k)\right]$ versus $k^{2}$, the interfacial widths can be determined. This is shown in figure 3.7 for the 10.8 at.\% $\mathrm{Fe}$ and 12.4 at.\% Mo samples. Since the photons are below in energy any of the $\mathrm{K}$ absorption edges, the $\mathfrak{R}(E)$ should consist only of resonant Raman scattering. The resonant Raman effect should contribute to $\Re_{\text {FeGe }}(6912 \mathrm{eV})$ for the 10.8 at.\% Fe sample but it should not significantly alter the elastic scattering (only when the energy is much closer to the edge). The scan of the 12.4 at.\% Mo sample at $7100 \mathrm{eV}$ is so far removed from the $\mathrm{K}$ edges that $\mathfrak{R}_{\text {MoGe }}(7100 \mathrm{eV})$ should be negligible. For the Fe-containing sample, the appropriate fitting region is $-0.35<k<0.50$ before the SNR worsens, whereas the SNR is sufficient to extend the fitting region to larger $k$ for the 12.4 at.\% Mo scan. The $2 \sigma_{w}$ are listed in table 3.2 and do not follow any apparent systematic behavior; in fact, for some sample the widths are unphysical. We will come back to these values after we have discussed an alternative approach to the large $k$ scattering. 

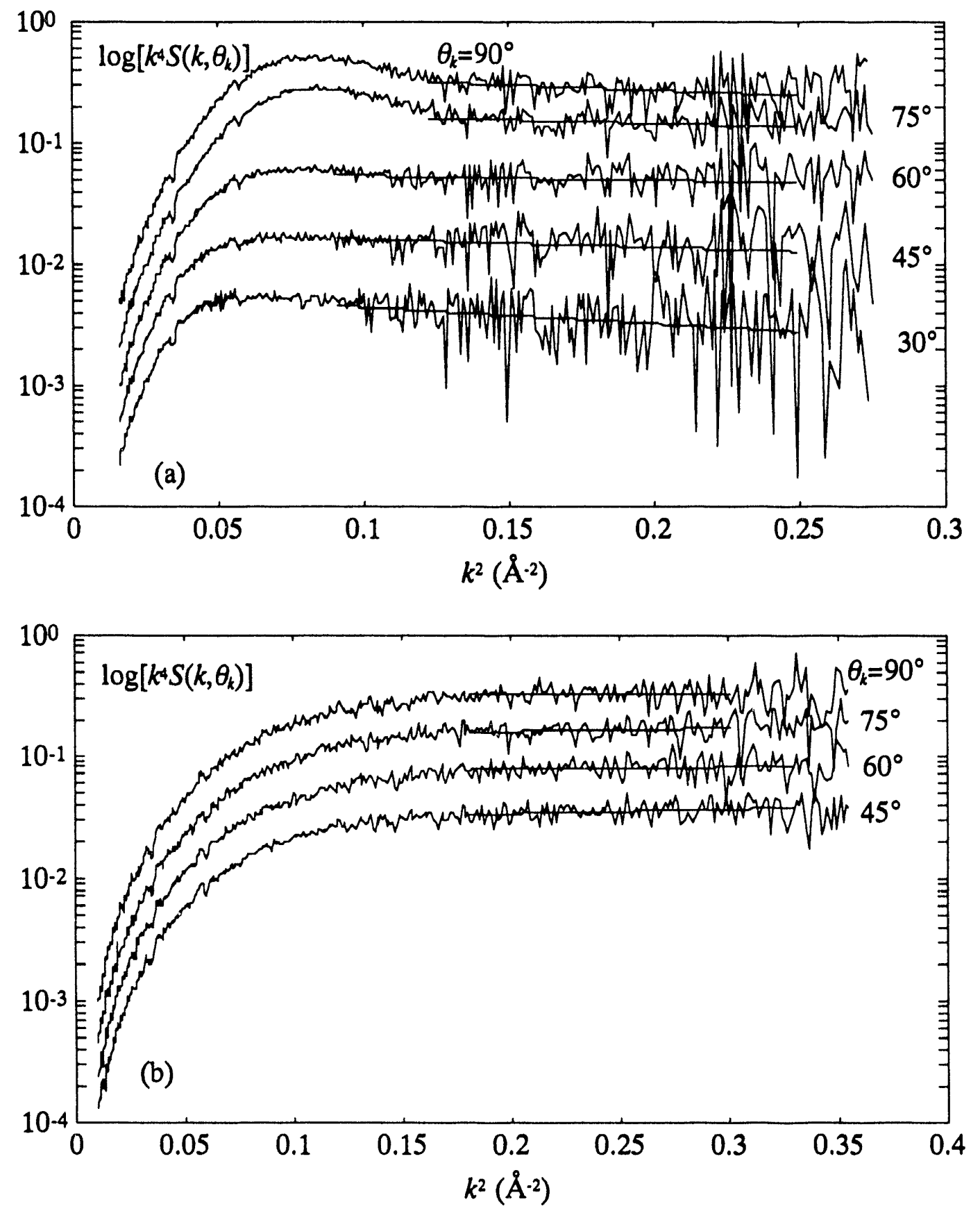

Figure 3.7. Ruland plots, $\log \left[k^{4} S\left(k, \theta_{k}\right)\right]$ versus $k^{2}$, for (a) 10.8 at.\% $\mathrm{Fe}$ and (b) 12.4 at.\% Mo. Plots offset for clarity. 
Table 3.2. Results of large $k$ fits to select samples for various $\theta_{k}$.

\begin{tabular}{|c|c|c|c|}
\hline $\begin{array}{c}\text { sample } \\
\text { fitting region }\end{array}$ & $\begin{array}{c}\text { polar angle } \\
\left(\theta_{k}\right)\end{array}$ & $\begin{array}{c}\alpha \\
\propto 1 / k^{\alpha}\end{array}$ & $\begin{array}{c}2 \sigma_{w} \\
(\AA)\end{array}$ \\
\hline \hline $\begin{array}{c}10.8 \text { at.\% Fe } \\
0.35<k<0.50\end{array}$ & 90 & 4.7 & 2.8 \\
\hline $0.35<k<0.50$ & 75 & 4.5 & 2.4 \\
\hline $0.35<k<0.50$ & 60 & 4.3 & 1.8 \\
\hline $0.35<k<0.50$ & 45 & 4.5 & 2.5 \\
\hline $0.35<k<0.50$ & 30 & 4.3 & 3.5 \\
\hline $\begin{array}{c}12.4 \text { at. \% Mo } \\
0.425<k<0.55\end{array}$ & 90 & 4.0 & 0.08 \\
\hline $0.425<k<0.55$ & 75 & 3.7 & $1.6 i$ \\
\hline $0.425<k<0.575$ & 60 & 3.8 & $1.3 i$ \\
\hline $0.425<k<0.575$ & 45 & 3.6 & $1.8 i$ \\
\hline
\end{tabular}

In a different approach to the diffuse transition between the two phases, Schmidt et al. ${ }^{13}$ have shown that the SAXS at large $k$ can decay at a rate greater than $k^{-4}$ and as great as $k^{-6}$ in the form of power law scattering. To see if a power-law relationship exists, it is instructive to create a log-log plot of the scattering. If there is a power-law at large $k$, then it should be observed as a linear region on the log-log plot. Figure 3.8 shows the oblique transmission results from the 10.8 at.\% $\mathrm{Fe}(E=6912 \mathrm{eV})$ and 12.4 at.\% Mo $(E=7100 \mathrm{eV})$ samples. Table 3.2 shows the power laws determined by extracting the slope from the log$\log$ plots. The large $k$ power-law does not change by more than $10 \%$ with changes in $\theta_{k}$ for a particular sample, so it is not clear if the changes are significant or not. From the Ruland plots, we find the interfacial widths are on the order of a few atoms, and for those power-laws less than 4 , they are imaginary. As mentioned previously, Schmidt has found 

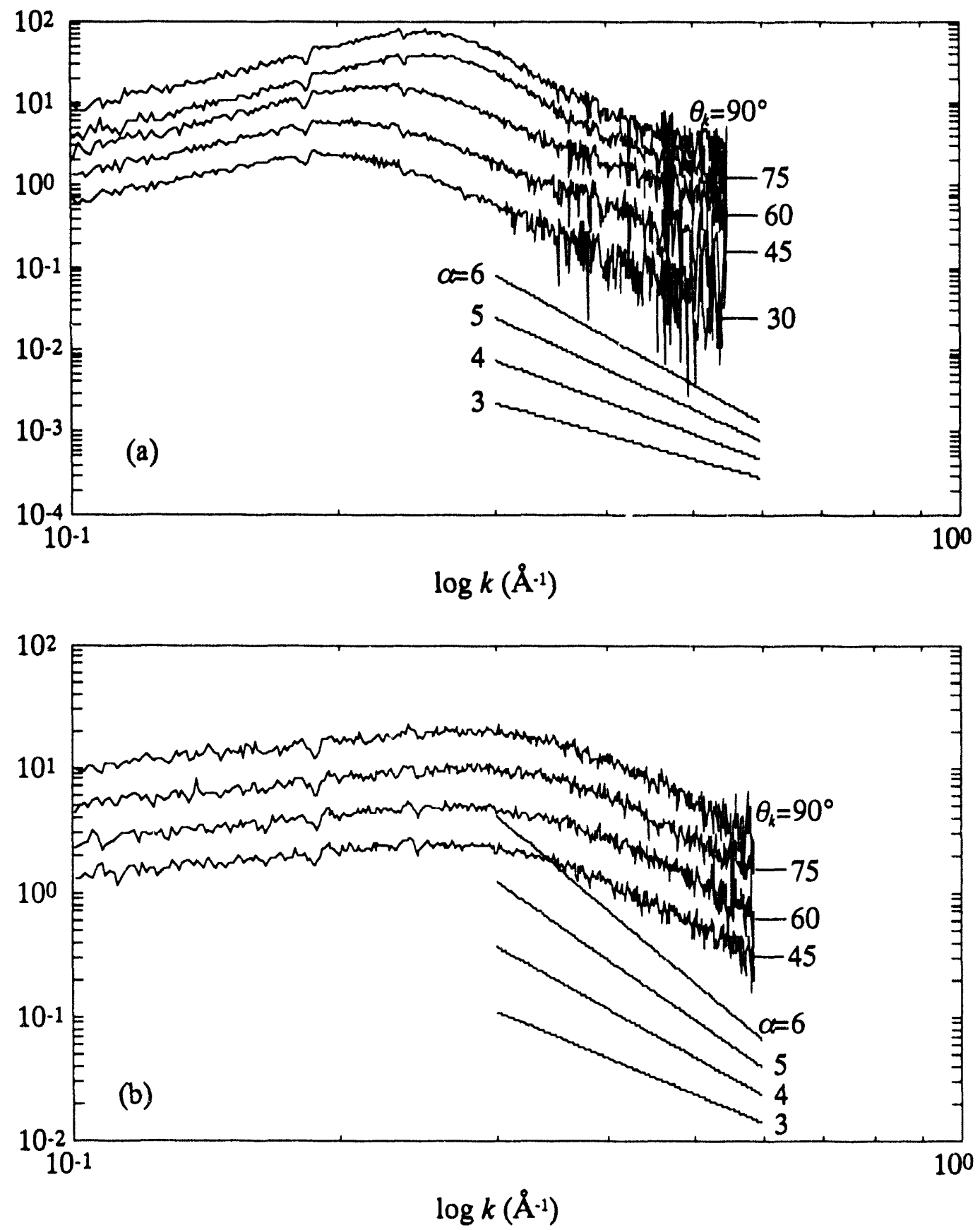

Figure 3.8. log-log plots of absolute-scaled scattering, $d \sigma_{\text {elas }}(\vec{k}, E) / d \Omega_{V}+\Re(E)$ (plots offset for clarity). For comparison, power-law plots for various exponents are included; these illustrate that a power-law close to 4 works well. (a) 10.8 at.\% $\mathrm{Fe}$ sample with $E=6912 \mathrm{eV}(200 \mathrm{eV}$ below the Fe K-edge). (b) 12.4 at.\% Mo sample with $E=7100 \mathrm{eV}$. The photon energy is far removed from any absorption edges in $(b)$, so $\Re(E)$ should be zero. It is probably close to zero in (a) as well. 
variations of up to $10 \%$ of a local power law from that fit over several decades in $k$. This provides a reasonable explanation for the imaginary interfacial widths for some of the scans $(\alpha<4)$.

To investigate the relationship between the interfacial widths and the power laws, we determined the interfacial width as a function of $\alpha$ for similar fitting regions in $k$ as that for our samples, shown in figure 3.9. The functions of course are not the same, but when one is fit to the other over such a small $k$-range $(0.4<k<0.60)$, they are essentially identical within the SNR of the data (see figure 3.9). For a $10 \%$ change in power-law, for example from $\alpha=4$ to $\alpha=4.4$, the interfacial width changes from 0 to $2.5 \AA$. Since the cylindrical correlation function (see Chapters 4 and 5) indicates that regions of similar electron density (i.e. "particles") are on the order of $10 \AA$ in any direction $\theta_{x}$ for either the Mo-Ge or Fe-Ge samples, the total interfacial width may consist of up to $50 \%$ of the particle (with the $\alpha=5$ power-law). Since we cannot measure the power-law scattering fall-off at large $k$ better than this $10 \%$, the meaning of the interfacial widths have little value. They do indicate, however, that we cannot quantitatively assess the density transition from particle to matrix. It is thus by no means clear that the interfaces are sharp between "particle" and matrix.

It appears as though the fall-off at large $k$ goes dpproximately as $k^{4}$ or, at most, $k^{5}$, which is consistent with a Ruland-type model. It should be noted that most theories on power-law scattering assume that the structure factor is isotropic. We have applied these models in an ad hoc way; that is, we have fit the scattering for a given $\theta_{k}$ to an isotropic scattering theory. From an empirical point of view, we find that the power-law scattering does not appear to depend significantly on the anisotropy.

Figure 3.10 illustrates how a constant background (e.g. resonant Raman scattering) can contaminate the power-law that is measured for a sample. In figure 3.10a, the log-log plot of scattering from the 6.5 at.\% Mo sample is shown, collected at $7100 \mathrm{eV}$ and 10803 

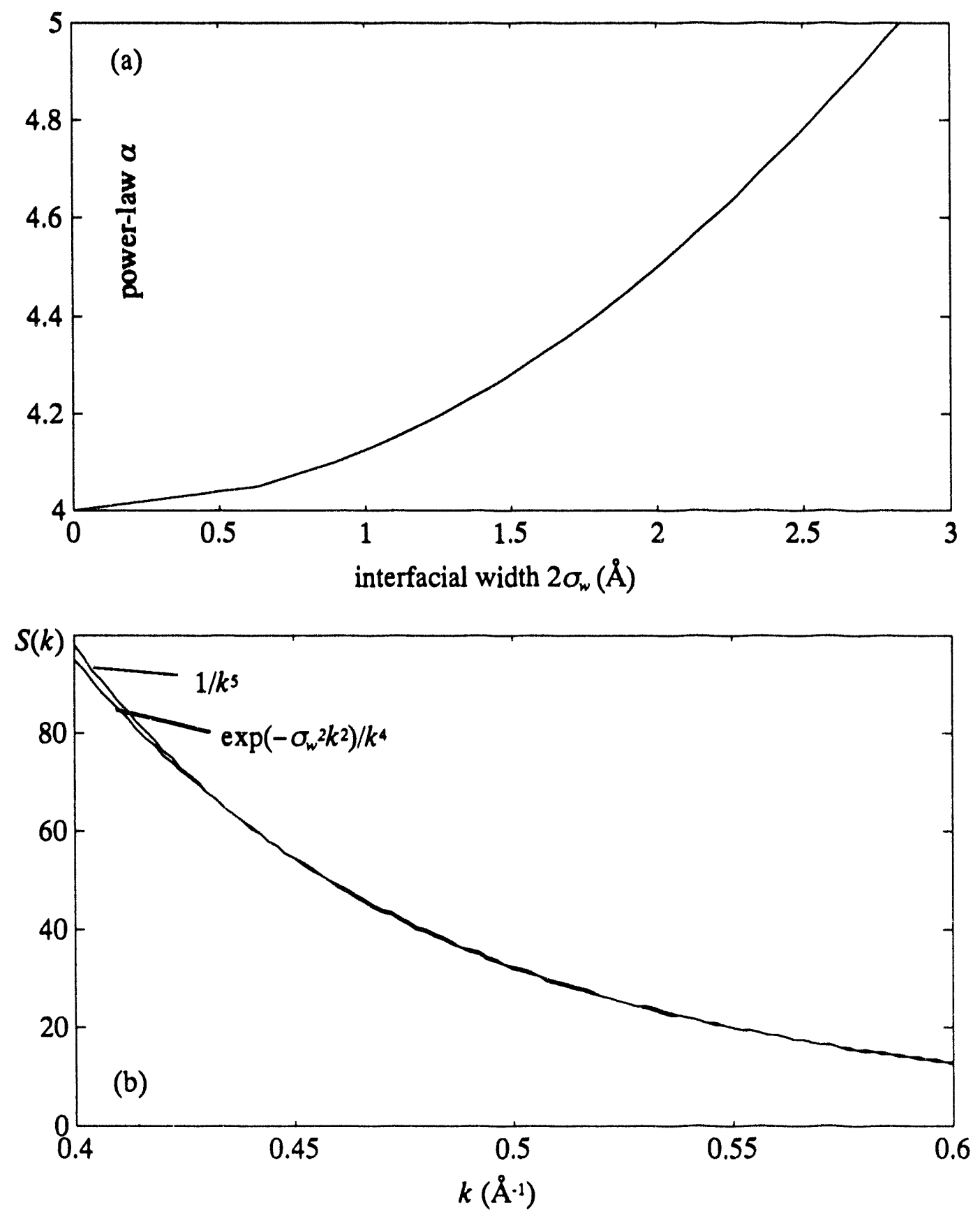

Figure 3.9. (a) Relationship between interfacial width $2 \sigma_{w}$ and power law $\alpha .1 / k^{a}$ data set created for $0.4<k<0.6$ and then fit to the interfacial width model to extract $2 \sigma_{w}$. (b) Comparison of power law $\alpha=5$ and the Porod law with a finite interfacial width. 

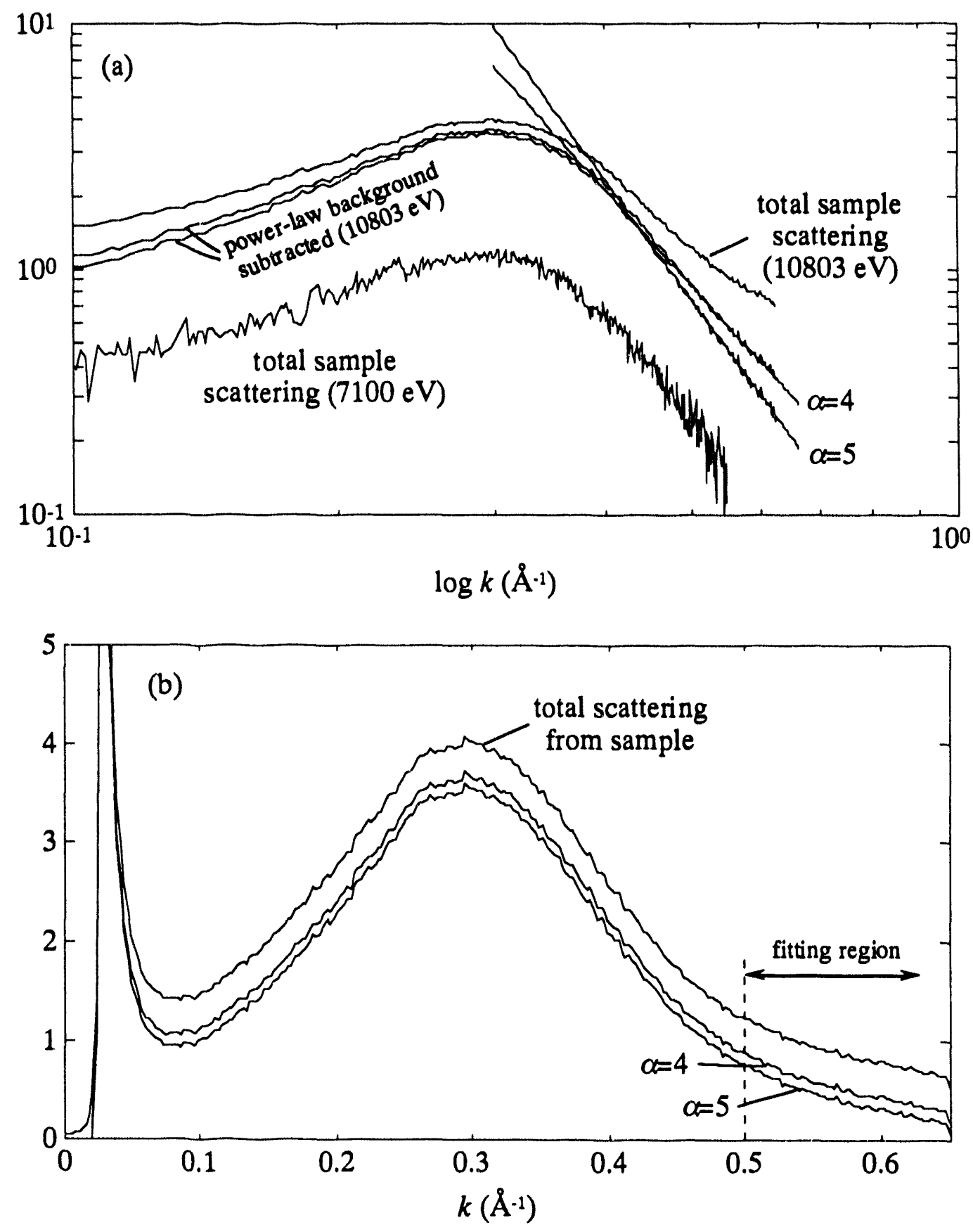

Figure 3.10. At $300 \mathrm{eV}$ below the $\mathrm{Ge} \mathrm{K}$-edge $(10803 \mathrm{eV})$, scattering at large $k$ from the 6.5 at.\% Mo sample does not exhibit a power-law close to 4 that is observed at $7100 \mathrm{eV}$. This is likely due to the presence of $\mathfrak{R}(10803 \mathrm{eV})$. With subtraction of different constants, however, the scattering can be fit to various power-laws, most notably $\alpha=4$ and 5 in the above. 
$\mathrm{eV}$ along with the necessary $\mathfrak{R}(E)$ subtracted to fit the $10803 \mathrm{eV}$ data to the elastic scattering $\alpha=4$ and 5 power-laws. It is clear that the power-law scattering at large $k$ is different at the two energies, which is due to the contamination of the elastic scattering signal by the resonant Raman scattering. For a fitting region $0.40<k<0.50 \AA^{-1}$, we have $\alpha=4.5$ and 3.4 for energies 7100 and $10803 \mathrm{eV}$, respectively.

Although not too surprising, I have found that the partial structure factors are fairly insensitive to the method of background subtraction, as long as it is performed in some consistent way. This has been the case for this work, when one of the PSFs is far greater than the other two, but it is not likely to be true in general. Figure 4.4 shows the PSFs for the 13.6 at.\% Fe sample calculated for various types of background subtraction, from fitting the high $k$-side of the SAXS maximum to power-law fits of $\alpha=4$ and 5 to aligning the energy-dependent scans to coincide at very large $k$. The method of background subtraction is important, however, when calculating the total elastic cross-section (Section 4.2) which defines the mean square fluctuation in electron density (Eq. 3.1.9). The elastic cross-section is determined by integrating the elastic scattering from 0 to $k \rightarrow \infty$, so an accurate subtraction of the $\Re(E)$ and extrapolation to $\infty$ with, for example, a power law is essential.

We have tried to fit the function $A+B / k^{\alpha}$ to the scattering patterns with a nonlinear least-squares routine. The results are inconclusive, however, since the relevant $k$ range that can be fit is less than a decade--limited by the large interparticle interference at small $k$ and the poor SNR at larger $k$. With such a small $k$-range, it is possible to fit various power-laws that agree quite nicely with the data yet are simply wrong. For example, consider the analytic function $1 / k^{4}$ presented in figure 3.11. By including a constant offset ( $5 \%$ of the maximum intensity in the fitting range) and random noise that increases with $k$, the best-fit power-law from a $\log -\log$ plot is $\alpha=3.65$. The greatest 


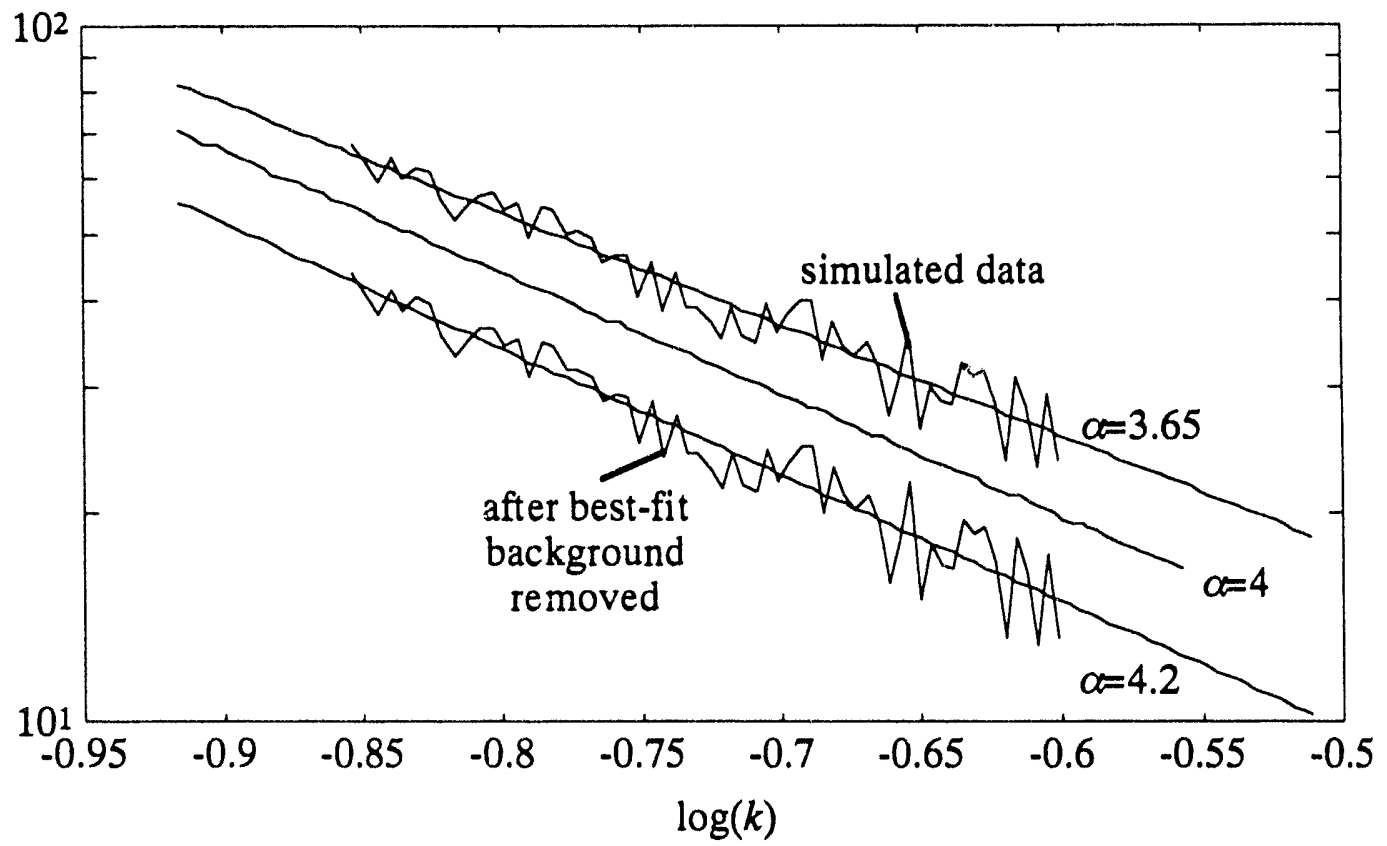

Figure 3.11. Simulated, noisy, large $k$ data that illustrates the difficulty in determining an accurate power-law $\alpha$ with such a small $k$ range. The simulated data follow an $\alpha=4$ power-law with an added constant of $5 \%$ of the largest intensity and random noise that increases with $k$. The fit to $A+\frac{B}{k^{a}}$ gives $\alpha=4.2$. The problem is with the limited $k$-range, which allows very similar sets of $\{A, B, \alpha\}$ to reproduce the data. 

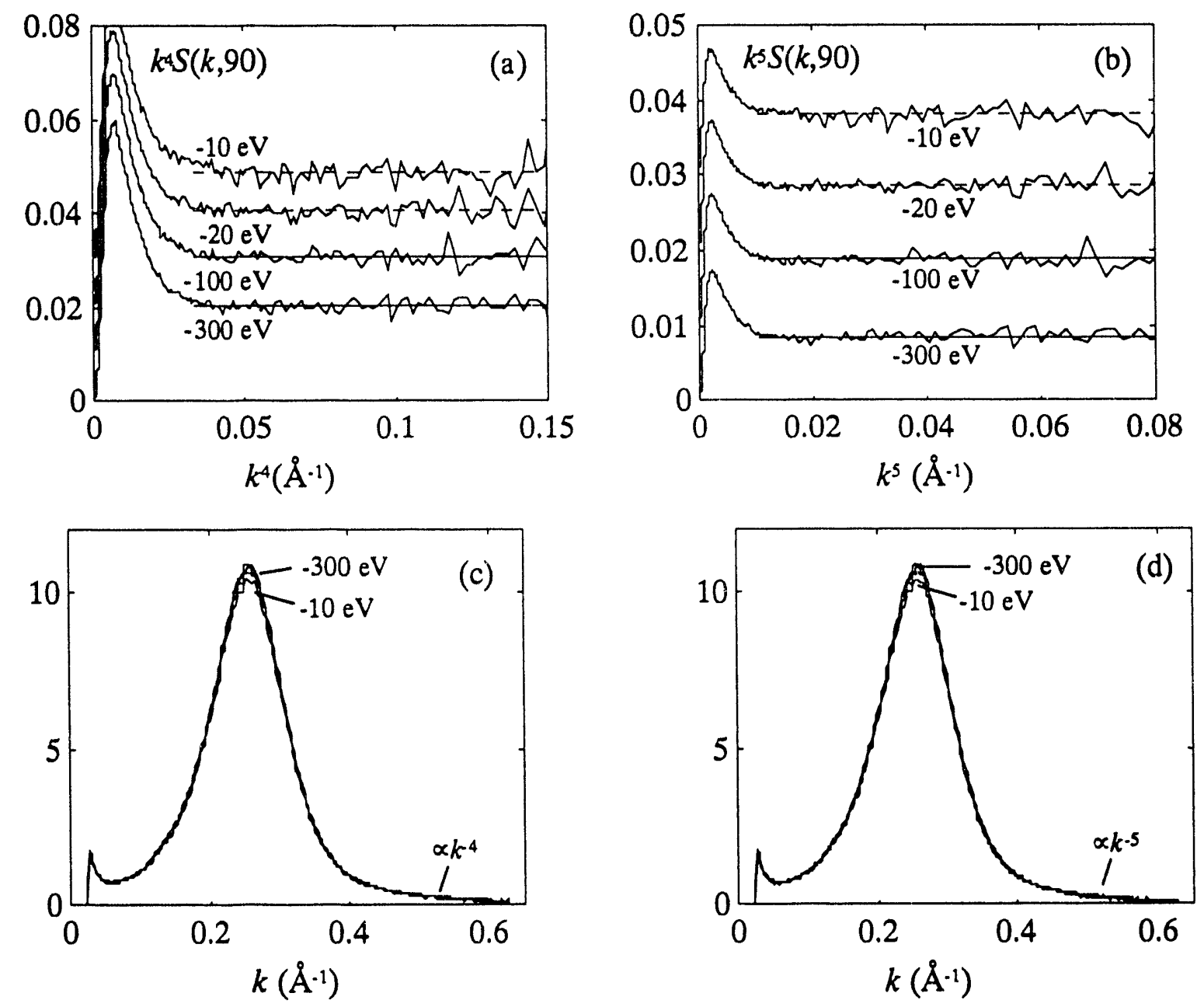

Figure 3.12. Background subtraction for the 13.6 at. $\% \mathrm{Fe}$ sample for $\theta_{k}=90^{\circ}$ for four energies below the Ge K-edge. (a) Porod plot (offset for clarity): $k^{4} r_{e}{ }^{2} S(k, 90)$ versus $k^{4}$ after subtraction of the $B\left(E, \theta_{k}\right)$. (b) $\alpha=5$ power law plot: $k^{5} r_{e}{ }^{2} S(k, 90)$ versus $k^{5}$ after subtraction of the $B\left(E, \theta_{k}\right)$. (c) $r_{e}{ }^{2} S(k, 90)$ with assumed $k^{-4}$ fall-off. (d) $r_{e}{ }^{2} S(k, 90)$ with assumed $k^{-5}$ fall-off. 
deviation from the $\alpha=4$ law is at the larger $k$ values in the fitting region, which are more susceptible to the constant offset. When fit to the function $A+B / k^{\alpha}$, the best-fit powerlaw is $\alpha=4.2$ (using a Simplex routine in MATLAB). With such a small $k$-range, the fit is extremely sensitive to any errors. This is a model case, but if there is a problem with imperfect window subtraction, for example, it becomes even more difficult.

The data presented in this work have been subtracted in the following way (similar to that by Maret et al. ${ }^{12}$ ). For a particular sample and oblique transmission angle, the elastic scattering is assumed to follow a power law. That is, it is fit to the large $k$ SAXS to remove background:

$$
\frac{d \sigma_{e l a s}\left(k, \theta_{k}, E\right)}{d \Omega_{V}}+\Re(E)=A\left(E, \theta_{k}\right) / k^{\alpha}+B\left(E, \theta_{k}\right)
$$

The $B$ is then removed from each scan, rendering $d \sigma_{\text {elas }}\left(k, \theta_{k}, E\right) / d \Omega_{v}$. The values of $B$ and $A$ (for $\alpha=4$ they are related to the "range of inhomogeneity parameter" or "Porod radius" often found in SAXS textbooks ${ }^{15}$ ) are only used for background subtraction and not for structural information; rather, the real-space cylindrical correlation function has been determined and provides a more sensitive means to extract structural information from an anisotropic system than the change in 'Porod radius' with $\theta_{k}$. Since it is not possible to unambiguously determine $\alpha$, the integrated intensities have been determined for two different $\alpha, \alpha=4$ and 5 to test the sensitivity of physical results to the choice of $\alpha$. Figure 3.12 demonstrates the two different power-law fits for a sample held at $\theta_{k}=90^{\circ}$ and energies below the Ge K-edge. Evidently, the fits are quite good for either power law. 
References for Chapter 3

${ }^{1}$ C.J. Sparks, Phys. Rev. Lett. 33, 262 (1974).

2P.H. Fuoss, P. Eisenberger, W.K. Warburton, and A. Bienenstock, Phys. Rev. Lett. 46, 1537 (1981).

3N.J. Shevchik, Phil. Mag. 35, 805, 1289 (1977).

${ }^{4}$ M. Rice, S. Wakatsuki, and A. Bienenstock, J. Appl. Cryst. 24, 598 (1991).

${ }^{5}$ See, for example, Section 14.5, B.E. Warren, $X$-Ray Diffraction (Addison-Wesley, Reading, Mass., 1969).

${ }^{6}$ S. Wakatsuki, K.O. Hodgson, D. Eliezer, M. Rice, S. Hubbard, N. Gillis, S. Doniach, and U. Spann, Rev. Sci. Instrum. 63, 1736 (1992).

7M. Rice and S. Wakatsuki, A User's Guide to the Small-Angle Scattering/Diffraction Data Acquisition System at SSRL, SSRL Publication PROD-UG-SAS 09/91-R01 (1991).

8V. Luzzati, Acta. Cryst. 13, 939 (1960).

${ }^{9}$ T.P. Russell, R.S. Stein, M.K. Kopp, R.E. Zedler, R.W. Hendricks, and J.S. Lin, The Application of a One-Dimensional Position Sensitive Detector to a Kratky SmallAngle X-ray Camera, Oak Ridge Laboratory Publication ORNL-TM-6678 (1978).

${ }^{10}$ T.P. Russell, J.S. Lin, S. Spooner, and G.D. Wignall, J. Appl. Cryst. 21, 629 (1988).

${ }^{11}$ O. Lyon and J.P. Simon, Phys. Rev. B 35, 5164 (1987).

${ }^{12}$ M. Maret, J.P. Simon, B. Boucher, R. Tourbot, and O. Lyon, J. Phys. Condens. Matter 4, 9709 (1992).

13P.W. Schmidt, D. Avnir, D. Levy, A. Hohr, M. Steiner, and A. Roll, J. Chem. Phys. 94, 1474 (1991).

${ }^{14}$ W. Ruland, J. Appl. Cryst. 4, 70 (1971). 
${ }^{15}$ See, for example, O. Glatter and O. Kratky, Small-Angle X-Ray Scattering (Academic Press, London, 1982). 


\section{Chapter 4}

\section{Amorphous $\mathrm{Fe}_{\mathrm{x}} \mathrm{Ge}_{1-\mathrm{x}}$ Results}

In this chapter we report the oblique and anomalous $\mathrm{x}$-ray scattering measurements for amorphous $\mathrm{Fe}_{\mathrm{x}} \mathrm{Ge}_{1-\mathrm{x}}$ and interpret the data in terms of scattering theory. The methods of differential anomalous scattering (DAS) as applied to amorphous components are well understood as are the basic tenets of small-angle $\mathbf{x}$-ray scattering. The combination of both theories along with the full differential cross-section as determined by the oblique measurements provides new incights to the structure of these films.

\subsection{Anisotropy and DAS Results}

Figure 4.1 shows the structure factor $S(\vec{k}, E)$ determined from the oblique transmission experiment for several $\mathrm{Fe}_{\mathrm{x}} \mathrm{Ge}_{1 . \mathrm{x}}$ samples. A strong anisotropy appears, observable from the change in the scattering peak with sample orientation. As the sample is tilted, the peak shifts inward to smaller $k$, monotonically decreasing with $\theta_{k}$. Although the existence of a peak in a SAXS data set is not necessarily a tell-tale sign of a particularly important lattice spacing or structure, it is at least a firm indication of welldefined medium-range distances. These data demonstrate that there exists a range of in- 


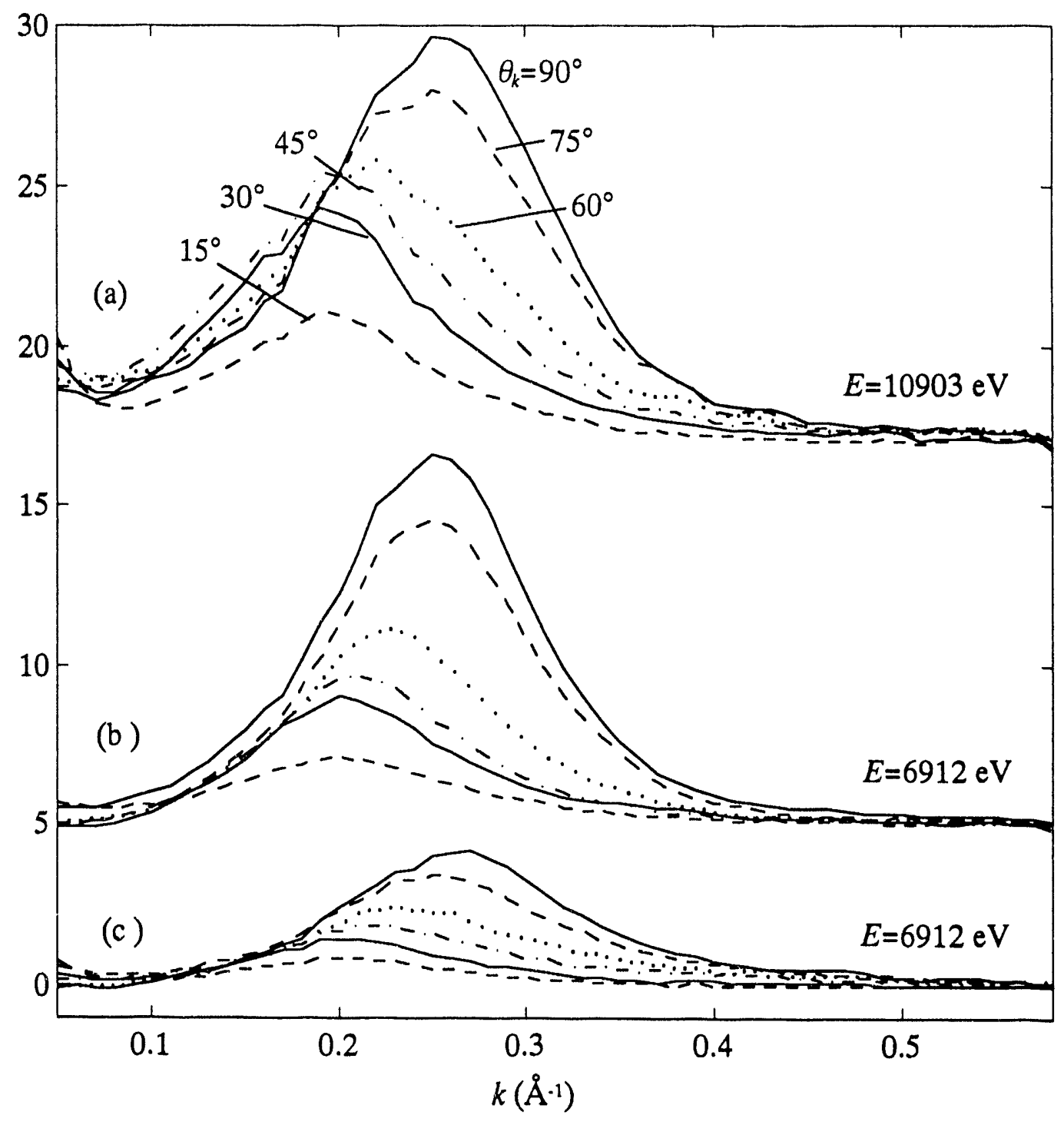

Figure 4.1. $r_{e}{ }^{2} S\left(k, \theta_{k}\right)\left(\mathrm{cm}^{-1}\right)$ for a-Fe $\mathrm{Fe}_{\mathrm{x}} \mathrm{Ge}_{1 \cdot \mathrm{x}}:$ (a) 18.2 at. $\% \mathrm{Fe}$, (b) 13.6 at. $\% \mathrm{Fe}$, and (c) 6.3 at. $\% \mathrm{Fe}$. In each case, as $\theta_{k}$ changes from $90^{\circ}$ to $0^{\circ}$, the SAXS peak monononically decreases in amplitude and shifts inward to smaller $k$. Data smoothed over an interval $\Delta k=0.02 \AA^{-1}$. 
plane correlations, which become gradually weaker and of smaller momentum transfer as the scattering vector shifts in orientation from the sample surface to the sample normal. All samples in this composition range $(x<0.33)$ illustrate the same behavior: as the scattering vector direction moves from sample surface to sample normal there is a monotonic decrease in scattering intensity and shift inward in peak position. However, by adding even more $\mathrm{Fe}$ to the alloy, the SAXS maximum disappears and we report no scattering for samples with 44 and 47 at.\% $\mathrm{Fe}$. The very existence of this peak and behavior with scattering vector direction has been difficult to understand in terms of model structures; consequently, Chapter 6 is spent discussing different models that give rise to its existence.

By changing the deposition conditions, films identical in composition can be grown to different states of phase separation. Figure 4.2 compares the scattering from samples of three compositions that have been prepared with two different target powers. By manipulating the target power, a host of variables in the vapor deposition process are altered (e.g. adatom thermal energy and deposition rate), so that it is not surprising that the samples exhibit different states of phase separation. Samples grown at 400 watts show a more "advanced" state of phase separation than those at 100 watts; that is, the SAXS maximum appears at a smaller magnitude of the scattering vector with greater amplitude. The greatest change is observed for the 6 at.\% Fe sample while only an intensity difference is apparent for the 25 and 27 at.\% $\mathrm{Fe}$ samples. The change in intensity for the 25 and 27 at.\% samples, however, may be due to the different overall film composition and not from the change in target power.

For all samples ( $<33$ at.\% $\mathrm{Fe}$ ) and oblique angles studied, only slight if any changes in $S(\vec{k}, E)$ are observed with energy near the Ge K-edge, but large changes exist at the Fe $\mathrm{K}$-edge. This result has previously been observed by Rice ${ }^{1}$ for amorphous M$\mathrm{Ge}(\mathrm{M}=\mathrm{Fe}$ and $\mathrm{W})$ at the $\mathrm{Fe}, \mathrm{W}$, and $\mathrm{Ge}$ edges and at normal incidence, but it appears to 


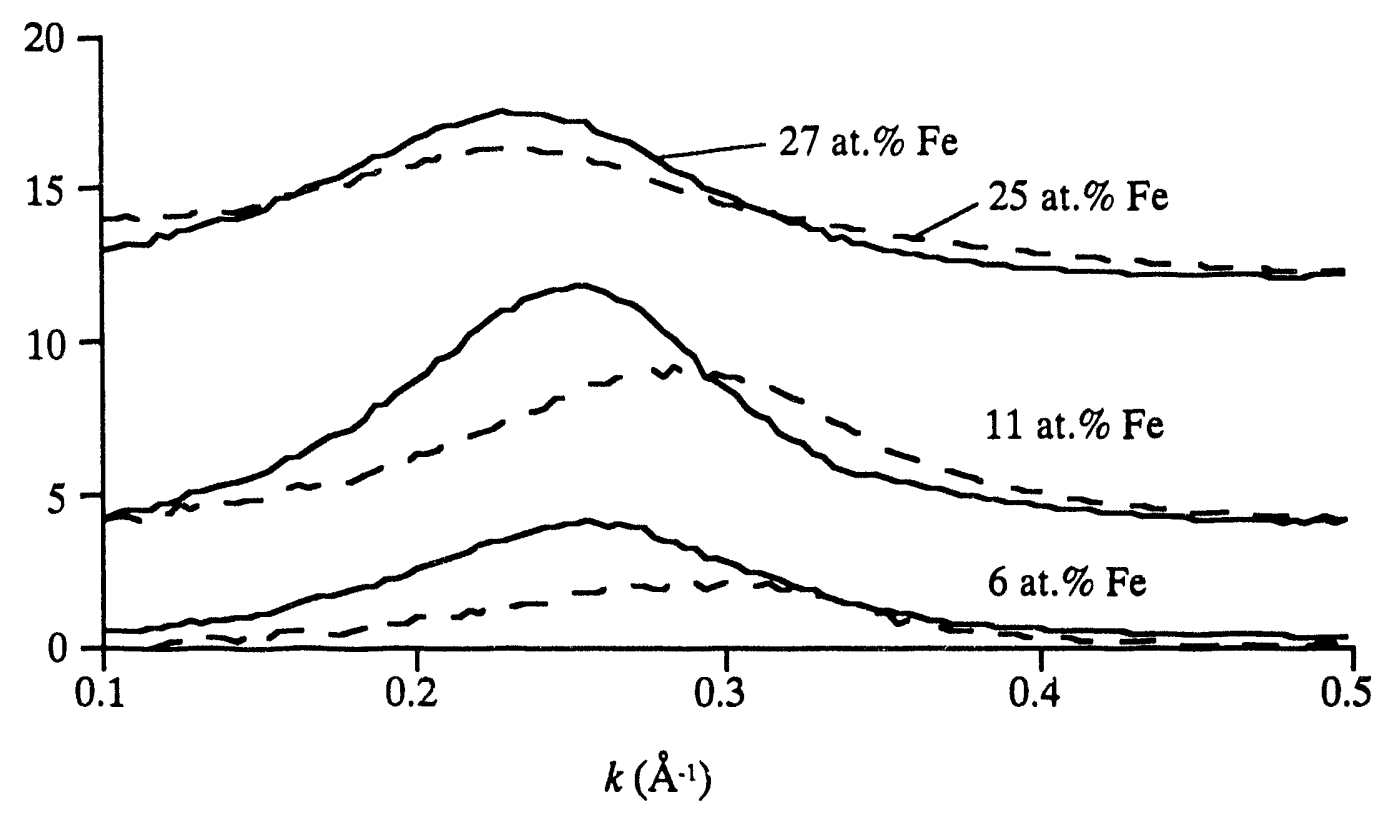

Figure 4.2. SAXS, $r_{e}{ }^{2} S(k, 90)\left(\mathrm{cm}^{-1}\right)$, as a function of composition and power delivered to the sputtering targets: - $400 \mathrm{~W}$ power; - - $100 \mathrm{~W}$. Data smoothed over an interval $\Delta k=0.02 \AA^{-1}$. 
be true for all oblique angles studied too. For differences of $16 \%$ in $f_{G e}(E)$, an approximate $4 \%$ intensity change of the main scattering peak is observed, whereas differences of $17 \%$ in $f_{F e}(E)$ lead to a $30 \%$ intensity shange The technique of DAS is employed to ascertain the origin of these observations. Figure 4.3 shows the changes at the $\mathrm{Ge}$ and $\mathrm{Fe} \mathrm{K}$-edges with respect to the change in $f^{\prime}$ for the 13.6 at.\% Fe sample. The Ge edge scans that are shown were obtained with a $\mathrm{Xe} / \mathrm{CO}_{2}$ detector gas--the scans that were collected with the $\mathrm{Ar} / \mathrm{CO}_{2}$ gas have a SNR that is significantly worse and consequently have not been used to determine species-specific information. The large change at the $\mathrm{Fe}$ edge is evidence that the $\mathrm{Fe}$ atoms are not distributed homogeneously. A small change at the Ge edge is observed, and since it is approximately an order of magnitude less intense than at the $\mathrm{Fe}$ edge, the $\mathrm{Ge}$ atoms, at least with respect to $\mathrm{Fe}$, are distributed considerably more homogeneously. To understand the degree of homogeneity on a more quantitative level, the PSFs must be determined.

The PSFs have been extracted using the Munro approach and are shown in figure 4.4 for the normal transmission case $\left(\theta_{k}=90^{\circ}\right)$ and different methods of background subtraction. The type of background subtraction employed seems to have little effect on the results, whether the scans are fit to power laws with $\alpha=4$ or 5 or are aligned at large $k \sim 0.55 \AA^{-1}$. There appears little, if any, Ge contribution to the total scattering pattern. Although the weighted-average PSFs (figure 4.3) indicate that there might exist a nonzero $S_{\text {FeGe }}$ and $S_{G e G e}$, the SNR and the large condition number lead to PSFs with little information about the Ge-Ge correlations except that they appear negligible compared to the $S_{F e F e}$ for all measured $k$. With $S_{F e G e}$ and $S_{G e G e}$ much smaller than $S_{F e F e}$, the observed scattering can be considered due to the inhomogeneity of $\mathrm{Fe}$ atoms. The most striking feature is the absence of any Ge-specific scattering throughout all oblique angles and composition range studied $(\mathrm{x}<0.33) . S(\vec{k}, E)$ appears comprised solely of $S_{\text {FeFe }}(\vec{k})$, which 

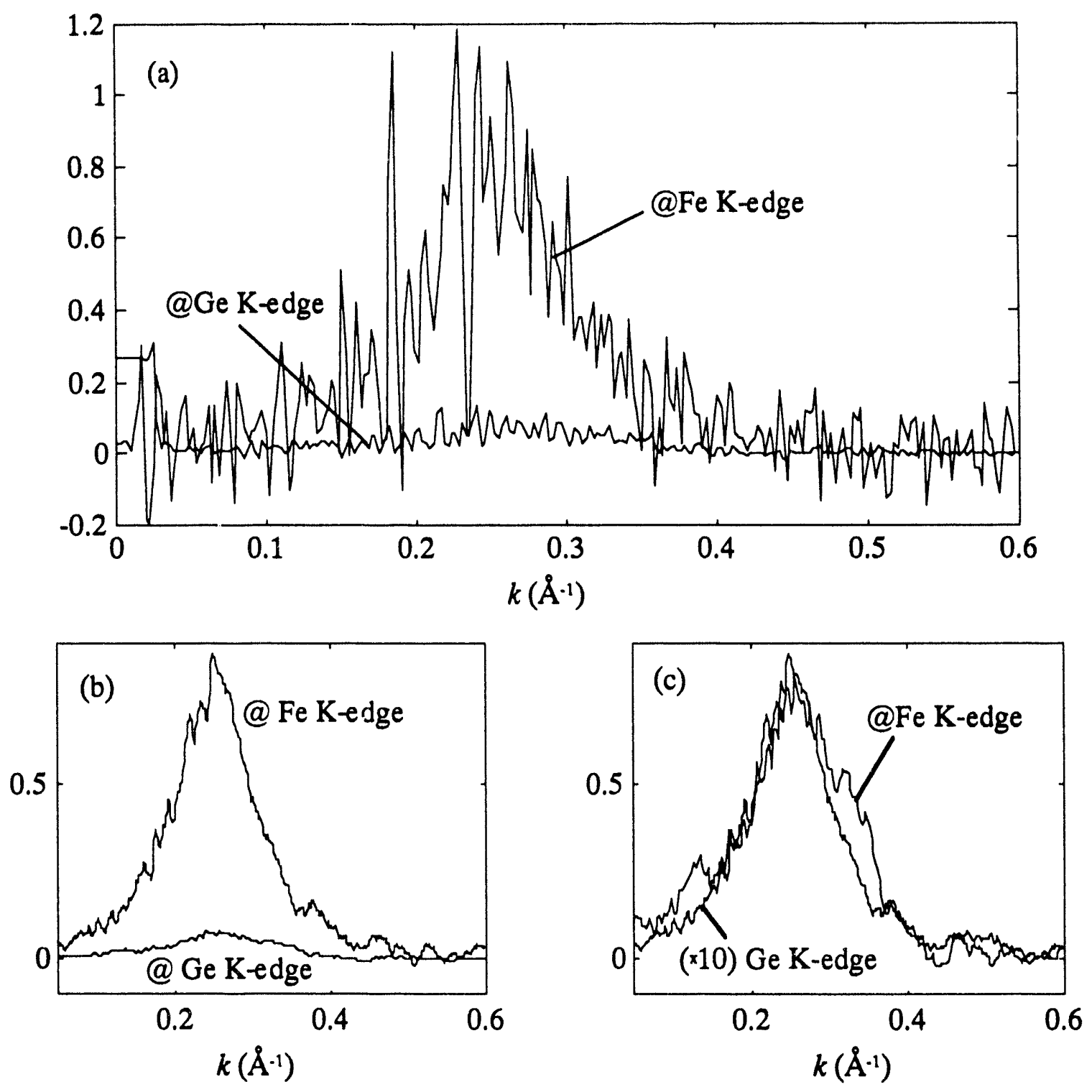

Figure 4.3. Weighted-average PSFs for 13.6 at. \% Fe sample. (a) Absolute changes in SAXS at the $\mathrm{Fe}$ and $\mathrm{Ge} \mathrm{K}$-edges, scaled by the relative change in $f(E)$. That is, $r_{e}^{2}\left(S_{E_{1}}(h, 90)-S_{E_{2}}(h, 90)\right) /\left(f^{\prime}\left(E_{1}\right)-f^{\prime}\left(E_{2}\right)\right)\left(\mathrm{cm}^{-1}\right)$. At the Ge edge, $E_{1}=-300$ and $E_{2}=-10$ $\mathrm{eV}$ below the edge, and $E_{1}=-200$ and $E_{2}=-10 \mathrm{eV}$ below the Fe edge. (b) Data smoothed over an interval $\Delta k=0.02 \AA^{-1}$. (c) Difference at the Ge edge scaled by an extra factor of 10. There is approximately an order of magnitude difference of homogeneity between the $\mathrm{Fe}$ and $\mathrm{Ge}$ atoms in this sample. Background subtracted with an assumed Porod $k^{-4}$ fit at large $k$. 

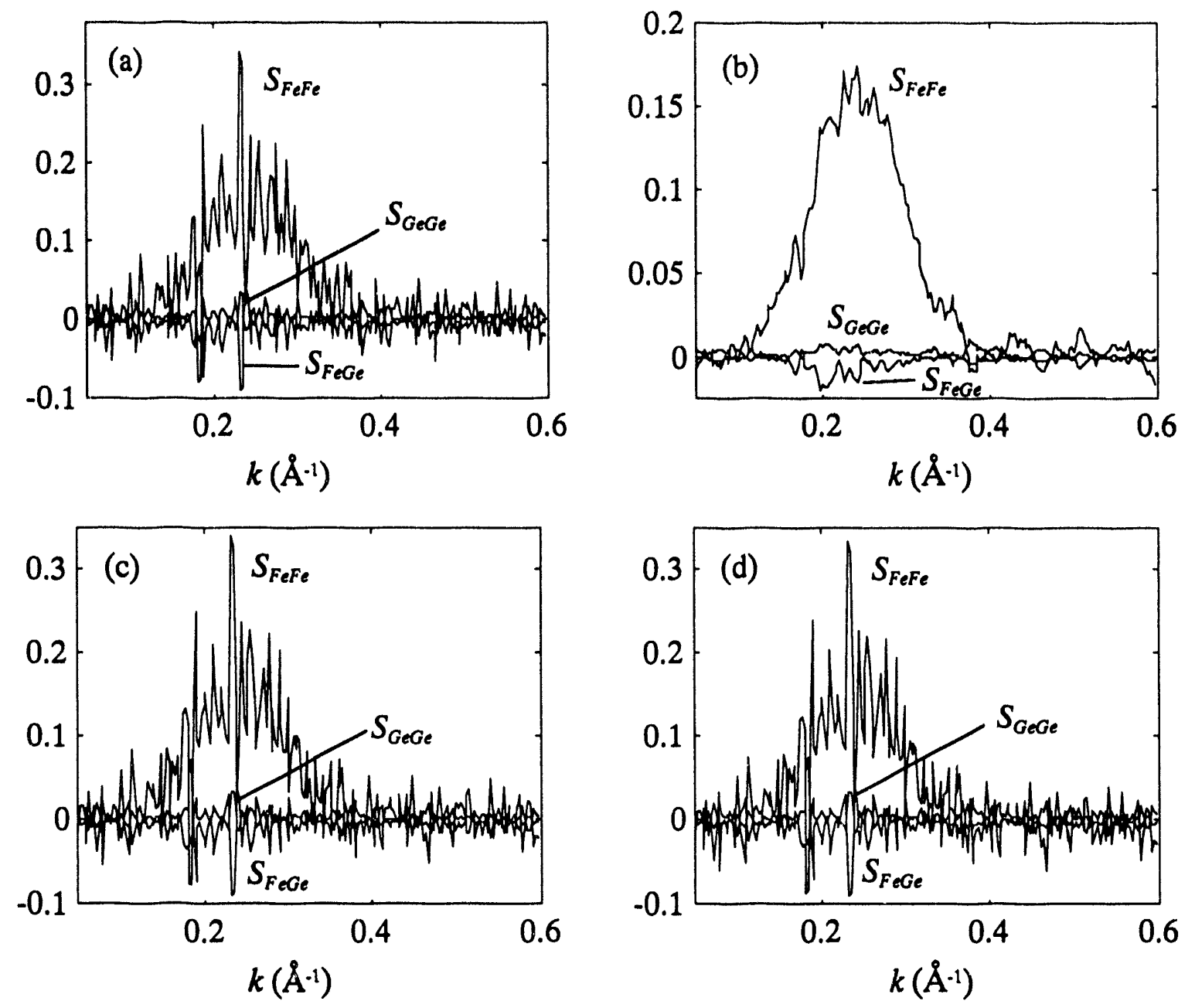

Figure 4.4. Normal incidence $\left(\theta_{k}=90^{\circ}\right)$ PSFs for the 13.6 at. $\%$ Fe sample determined with the Munro method (scaled by $r_{e}^{2}$ and in units $\mathrm{cm}^{-1}$ ). (a) PSFs from raw data with scans from energies $E_{1}=6912$ and $E_{2}=7102 \mathrm{eV}$ subtracted, energies $E_{3}=10803$ and $E_{4}=11093 \mathrm{eV}$ subtracted, and energy $E_{5}=6912 \mathrm{eV}$. Background removed with a Porod $k^{4}$ fit. (b) PSFs from (a) smoothed over an interval $\Delta k=0.02 \AA^{-1}$. (c) PSFs determined with the same method and energies as in (a) but with the background removed with an assumed $k^{-5}$ fit. (d) PSFs determined as in (c) but with the background removed by assuming the coherent scattering is 0 for $k>0.55$ for all energies and scans. Within the SNR, the PSFs appear identical and independent of the precise method of background subtraction. The SNR and large matrix condition numbers lead to PSFs that cannot isolate the Ge-Ge and Fe-Ge correlations that are observed in the weighted-average PSFs (figure 4.3b). 
must also be responsible for the anisotropy or change in correlation with film orientation. This result also implies that, for all samples, voids do not contribute appreciably to the SAXS. The concentration of voids appears negligibly small since any voids would greatly influence the scattering. The difference in electron density between regions of an amorphous phase and voids would be far greater than between two solid phases and would be easily observed as a non-zero $S_{\text {GeGe }}(\bar{k})$.

\subsection{Mean Square Fluctuation in Effective Electron Density}

As mentioned previously in Chapter 3, a general parameter that is commonly used in SAXS studies is the mean-square fluctuation in electron density, $\left\langle\eta^{2}\right\rangle$. With the anomalous dispersion relations, $\left\langle\eta^{2}\right\rangle$ becomes the mean-square fluctuation in effective electron density, $\left\langle\eta^{2}(E)\right\rangle$, as noted in Eq. 3.1.9. It is proportional to the elastic crosssection, also known as the integrated intensity or invariant in different textbooks. We start this section by reporting the mean-square fluctuation in effective electron density for several Fe-Ge samples. Since the transition width between particle and matrix cannot be unambiguously determined from these experiments (see Section 3.6), it appears particularly difficult to extract useful information from the $\left\langle\eta^{2}(E)\right\rangle$. This is not the case, however, and we will argue that it is possible, albeit with assumptions, to identify the composition of the phase-separating species.

\section{2.a Calculation and Values}

In practice, $\left\langle\eta^{2}(E)\right\rangle$ is calculated in 2-dimensions with an assumed azimuthal symmetry. That is, the integral in Eq. 3.1.9 is performed by integrating each radial scan in $k$ (for a given polar angle $\theta_{k}$ ) and then over $\theta_{k}$ : 


$$
\frac{\left\langle\eta^{2}(E)\right\rangle}{n_{G e}}=\frac{1}{\left(n_{G e} t\right) A r_{e}^{2} 2 \pi^{2}} \int_{0}^{\pi / 2}\left[\int_{0}^{\infty} \frac{d \sigma}{d \Omega}\left(k, \theta_{k}, E\right) k^{2} d k\right] \sin \theta_{k} d \theta_{k} .
$$

The integral over $k$ is performed in the usual way: the contribution from 0 to $k_{\min }$ summed with a triangular approximation, the data from $k_{\min }$ to $k_{\max }$ integrated with the trapezoidal rule, and the contribution from $k_{\max }$ to $\infty$ included by analytically integrating the $\alpha$ power law:

$$
\int_{0}^{\infty} \frac{d \sigma}{d \Omega}(\vec{k}, E) k^{2} d k \sim \frac{1}{2}\left|\vec{k}_{\min }\right|^{3} \frac{d \sigma}{d \Omega}\left(\vec{k}_{\min }, E\right)+\int_{k_{\operatorname{mbn}}}^{k_{\max }} \frac{d \sigma}{d \Omega}(\vec{k}, E) k^{2} d k+\frac{d \sigma}{d \Omega}\left(\vec{k}_{\max }, E\right) \frac{\left|\vec{k}_{\max }\right|^{3}}{(\alpha-3)}
$$

The contribution of the triangular approximation to the total integrated intensity is usually less than $0.1 \%$, but the contribution from the power law extrapolation can be as great as 20-30\%, which is due in large part to the finite $k$-range sampled. The value of $\left\langle\eta^{2}(E)\right\rangle$ is thus largely dependent on the quality of the $1 / k^{a}$ extrapolation and has been entirely excluded in some analyses, ${ }^{2}$ where a relative comparison between scans was desired. In our case, the fit to a power law can be quite good for $4<\alpha<5$, depending on the fitting region in $k$ (see Section 3.6). As mentioned previcusly, the contribution to the integral is determined for two different cases ( $\alpha=4$ and 5), which should span the range of possible $\alpha$ observed in these films. The integral over the polar angle is performed with a 7-point Simpson's rule. $\theta_{k}=0^{\circ}$ is not experimentally accessible but is not necessary either in order to calculate $\left\langle\eta^{2}(E)\right\rangle$, since the $\sin \theta_{k}$ term in the integrand forces the $\theta_{k}=0^{\circ}$ contribution to 0 . The measurements at $\theta_{k}=90,75,60,45,30$, and $15^{\circ}$ appear sufficient to adequately sample the anisotropy; Section 4.4 addresses this issue in more detail, where the cylindrical distribution function is calculated from an expansion in spherical harmonics. 
Figure 4.5 depicts the relationship between $\left\langle\eta^{2}(E)\right\rangle / n_{G e}$ and $\left|f_{F e}(E)\right|^{2}$ for several samples at the Fe K-edge and the two different power law extrapolations. Since there is no change in $\left\langle\eta^{2}(E)\right\rangle / n_{G e}$ with energy at the Ge K-edge (consistent with DAS results), it is not shown; we will come back to this null result later, however. $n_{G e}$ is used as a means to normalize $\left\langle\eta^{2}(E)\right\rangle$ to the sample thickness. Since the thickness is not known precisely, yet the absorption edge-jump measurements (Section 2.3) provide $n_{G e} t$, a factor of $n_{G e}$ is explicitly included in $\left\langle\eta^{2}(E)\right\rangle$. Nothing has been assumed about the densities in the sample up to this point. The different power-law extrapolations can change the overall mean-square fluctuation in density, in some cases up to $20 \%$. This is due to the different assumptions that are made about the phase separation. For sharp boundaries between regions of two different electron densities, as one might expect from distinct nuclei, the mean-square change in electron density will be larger than that from two regions of the same difference in electron density but with diffuse boundaries (e.g. early stages of spinodal decomposition).

Section 3.6 pointed out that we. veen unable to unambiguously define the size of the transition width between regions of different electron density. With the limited SNR at large $k$ (less than a decade), it is not possible to determine an accurate transition width from the Ruland plots; equivalently, we can fit an $\alpha=4$ (sharp transition) or an $\alpha=5$ (diffuse transition) power-law to the data. As mentioned in Section 3.6, with $\alpha=5$ the transition widths approach $\sim 40-50 \%$ of the regions of like electron density ("particle"), since these regions are no larger than $~ 10 \AA$ (see Section 4.3). In what follows, two models are discussed. In the first case, we assume the interfaces are sharp and employ the $\left\langle\eta^{2}(E)\right\rangle$ that are obtained with the Porod law. Alternatively, in the second case, we focus on a finite transition width that occupies a significant volume fraction of the sample. In either case, it is possible to determine the compositions of the 

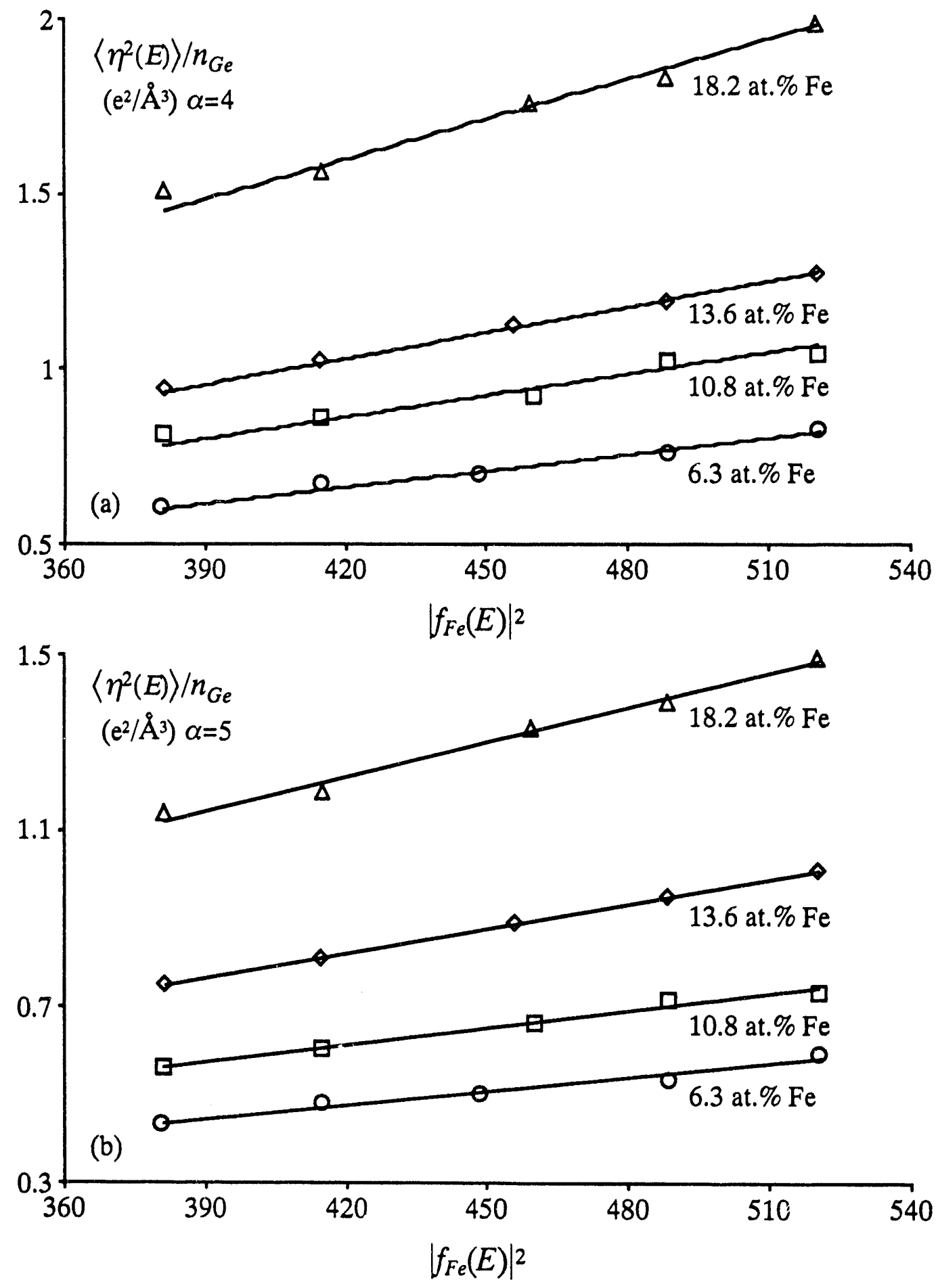

Figure 4.5. Mean-square fluctuation of the effective electron density as a function of the film composition $\mathrm{x}$ and different power-law fall-off at large $k$. (a) An assumed Porod $k^{4}$ fall-off (sharp transition between regions of different electron density); (b) an assumed $k^{-5}$ fall-off (finite transition width). 
phase-separating species. These two extreme examples allow us to place limits on the computed compositions, since the actual transition widths will probably vary between these two examples.

\section{2.b Sharp Transition}

If the samples are phase-separated into regions of well-defined electron densities with sharp, abrupt interfaces, then the large $k$ scattering obeys the $k^{4}$ Porod law. The mean-square fluctuation in electron density, for a two-phase system, can be explicitly written as

$$
\left\langle\eta^{2}(E)\right\rangle=c\left(\rho_{p}-\rho_{0}\right)^{2}+(1-c)\left(\rho_{q}-\rho_{0}\right)^{2}
$$

with $c$ the volume fraction of $F e_{p} G e_{1-p}$ with effective electron density $\rho_{p}$ and similarly for $(1-c)$ and $F e_{q} G e_{1-q}$. Since the previous section concludes that the contribution to the SAXS from voids is negligible, they have not been included in Eq. 4.2.3. (If voids are included, it is easy to show after some manipulation that they drop out of the calculation.) Previously, Rice et al. ${ }^{3}$ used a two-phase model to interpret the anomalous scattering from amorphous films. The approach developed here is identical, except that the anisotropy must be included. To see how this is related to the anomalous $x$-ray scattering experiments, the effective electron densities $\rho_{p}$ and $\rho_{q}$ can be written as linear combinations of the scattering factors for each element. For example, the phase of stoichiometry $F e_{p} G e_{1-p}$ has an electron density

$$
\rho_{p}(E)=n_{F e}^{p} f_{F e}(E)+n_{G e}^{p} f_{G e}(E)
$$


where $n_{F e}^{p}$ and $n_{G e}^{p}$ are the number densities of Fe and Ge atoms, respectively, in $F e_{p} G e_{1-p}$. By expanding Eq. 4.2.3 in terms of the scattering factors and number densities,

$$
\left\langle\eta^{2}(E)\right\rangle=c(1-c)\left|\left(n_{F e}^{p}-n_{F e}^{q}\right) f_{F e}(E)+\left(n_{G e}^{p}-n_{G e}^{q}\right) f_{G e}(E)\right|^{2}
$$

As noted, there is no resolvable change in $\left\langle\eta^{2}(E)\right\rangle$ with energy at the Ge K-edge while there are large changes with energy near the Fe K-edge. This implies $n_{G e}^{p}=n_{G e}^{q}$. In addition, we assume one endpoint is a-Ge (i.e. $p=0$ or $n_{\mathrm{Fe}}^{p}=0$ ) and hence rewrite Eq. 4.2 .5 as

$$
\left\langle\eta^{2}(E)\right\rangle=\frac{x}{1-x} n_{F e}^{q} n_{G e}\left(1-\frac{x}{1-x} \frac{n_{\sigma_{e}}}{n f_{e}}\right)\left|f_{F_{e}}(E)\right|^{2}
$$

where the volume fraction $c$ is expressed in terms of the measured atomic fraction $\mathbf{x}$ and $n_{G e}=n_{G e}^{p}=n_{G e}^{q}$. There could easily be $1-2 \%$ solubility of Fe in a-Ge, but since we observe the SAXS with the addition of only 6.3 at.\% Fe, the assumption of one endpoint consisting strictly of a-Ge seems reasonable.

With $\left\langle\eta^{2}(E)\right\rangle / n_{G e}, f_{F e}(E)$, and $\mathrm{x}$ measured in the ASAXS and absorption experiments, a linear fit of $\left\langle\eta^{2}(E)\right\rangle / n_{G e}$ to $\left|f_{F e}(E)\right|^{2}$ allows one to find the slope, $\frac{x}{1-x}\left(n_{F e}^{q}-\frac{x}{1-x} n_{G e}\right)$, with the constraint that the $y$-intercept equal 0 . Thrin, from the slope, $n_{F e}^{q}$ can be determined for a given $n_{G e}$ (or vice-versa), and thus the atomic fraction $q$ of the phase $\mathrm{Fe}_{q} \mathrm{Ge}_{1-q}$ determined:

$$
q=\frac{n_{F e}^{q}}{n_{G e}+n_{F e}^{q}}
$$




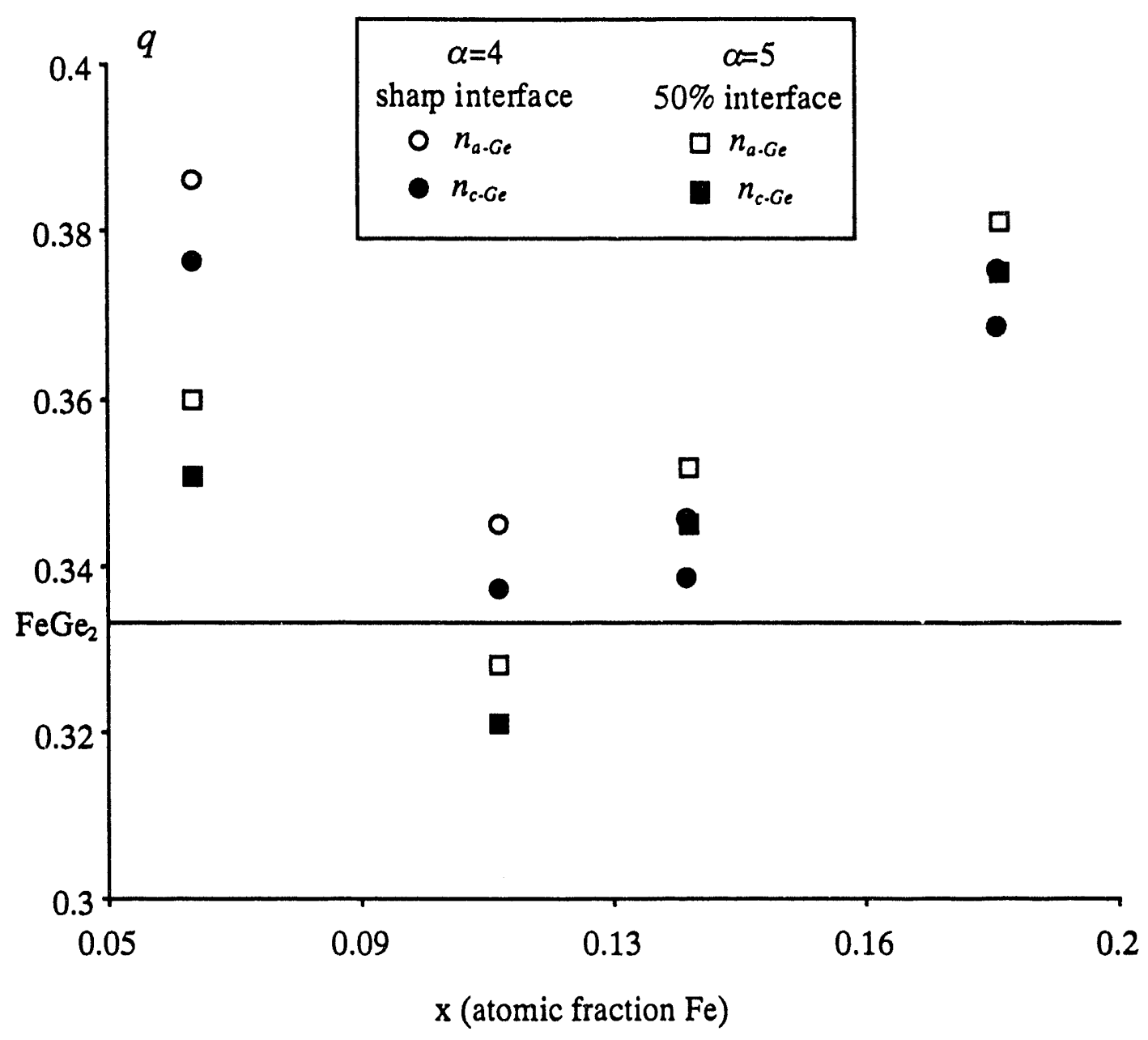

Figure 4.6. Endpoint compositions $q$ determined from the mean-square fluctuation in effective electron density for four different $\mathrm{Fe}-\mathrm{Ge}$ samples, with assumed number densities of Ge that are equal to that for c-Ge and $95 \%$ of c-Ge (a-Ge). For the theory of a sharp transition between the two-phase regions, $\left\langle\eta^{2}(E)\right\rangle / n_{G e}$ is determined with the Porod law extrapolation. For the theory of a $50 \%$ transition width, $\left\langle\eta^{2}(E)\right\rangle / n_{G e}$ is determined with a $k^{5}$ law. 


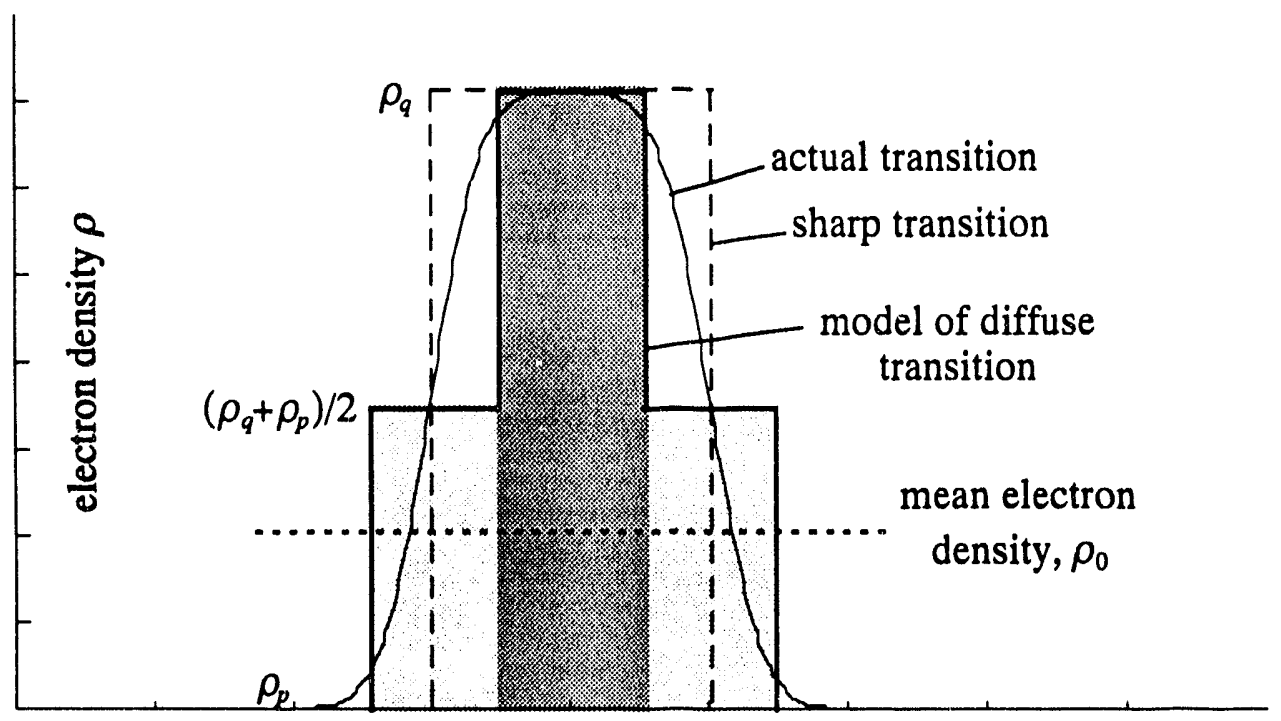

Figure 4.7. Diffuse interface model for two-phase system. The electron density of the interface is approximated as the average electron density between the two neighboring regions. 
The evident linear relationship between $\left\langle\eta^{2}(E)\right\rangle / n_{G e}$, calculated for $\alpha=4$, and $\left|f_{F e}(E)\right|^{2}$ shown in figure $4.5 \mathrm{a}$, illustrate that there is good agreement with the two-phase approximation of Eq. 4.2.6. For number densities of $95 \%$ of, and the crystalline value for, crystalline $\mathrm{Ge}$, the different possible endpoints $q$ are shown in figure 4.6. The results are convincing; for all samples studied, $q$ ranges from $\sim 34$ at. $\% \mathrm{Fe}$ to $\sim 38$ at. $\% \mathrm{Fe}$.

\section{2.c Broad Transition}

Consider the other extreme to the previous example. Suppose that the transition width occupies a large fraction of one of the phases; it was shown in Section 3.6 that this region may be on the order of $50 \%$ of the particle for power laws with $\alpha=5$. As an approximation to the electron density of the transition region, which in theory should vary continuously from $\rho_{p}$ to $\rho_{q}$, let the average of $\rho_{p}$ and $\rho_{q}$ occupy this region. Figure 4.7 illustrates such a three-phase model. If the volume fraction of material that has electron density $\rho_{q}$ is $c / 2$ and the volume fraction of electron density $\rho_{p}$ is $1-c$, then the overall electron densities must be related by

$$
\frac{c}{2} \rho_{q}+(1-c) \rho_{p}+\frac{c}{2}\left(\frac{\rho_{q}+\rho_{p}}{2}\right)=\rho_{0}
$$

The mean square fluctuation in electron density can be analytically written as:

$$
\left\langle\eta^{2}(E)\right\rangle=\frac{c}{2}\left(\rho_{q}-\rho_{0}\right)^{2}+(1-c)\left(\rho_{p}-\rho_{0}\right)^{2}+\frac{c}{2}\left(\frac{\rho_{q}+\rho_{p}}{2}-\rho_{0}\right)^{2} .
$$

With the electron densities expanded in terms of the atomic scattering factors and number densities and the volume fraction $c$ in terms of the atomic fraction $x$, we have 


$$
\left\langle\eta^{2}(E)\right\rangle=\frac{x}{1-x} n_{G e}\left(\frac{5}{b} n_{F e}^{q}-\frac{x}{1-x} n_{G e}\right)\left|f_{F e}(E)\right|^{2} .
$$

As in the previous example, the slope can be determined in Eq. 4.2.10, shown in figure $4.5 \mathrm{~b}$, which allows us to calculate the atomic fraction $q$ given a number density for a-Ge. For this model of a broad transition between the two phases, the $\left\langle\eta^{2}(E)\right\rangle / n_{G e}$ must be calculated with the $\alpha=5$ power law. The different possible endpoints $q$ are shown in figure 4.6 for two different number densities for a-Ge. For all samples studied, $q$ ranges from $\sim 32$ at. $\% \mathrm{Fe}$ to $\sim 38$ at. $\% \mathrm{Fe}$.

These two models are two extreme examples of the type of interfacial region that we suspect exist in these samples. Although we are unable to assess accurately the interfacial structure, it appears that, regardless of the interface, the composition of the regions that are somewhat removed from the interfacial regions can be determined. In this case, it appears that there is phase separation into a-Ge and an Fe-Ge intermetallic, close in stoichiometry to an amorphous $\mathrm{FeGe}_{2}$.

\subsection{Cylindrical Correlation Functions (CCFs)}

It is difficult to interpret the anisotropy from the scattering patterns alone, since the scaltering along a given polar angle $\theta_{k}$ is given by the Fourier transform of the projection of the real-space pair correlation function onto the plane defined by $\theta_{k}$. Any robust understanding, especially of an anisotropic noncrystalline system, thus relies on determining the electron density pair correlation function. From the vapor deposition process itself, the pair correlation function is likely to exhibit an "in-plane" isotropy with an axis of cylindrical symmetry in the direction of growth. The appropriate function in this case is the cylindrically symmetric pair correlation function. ${ }^{4}$ Such functions have 


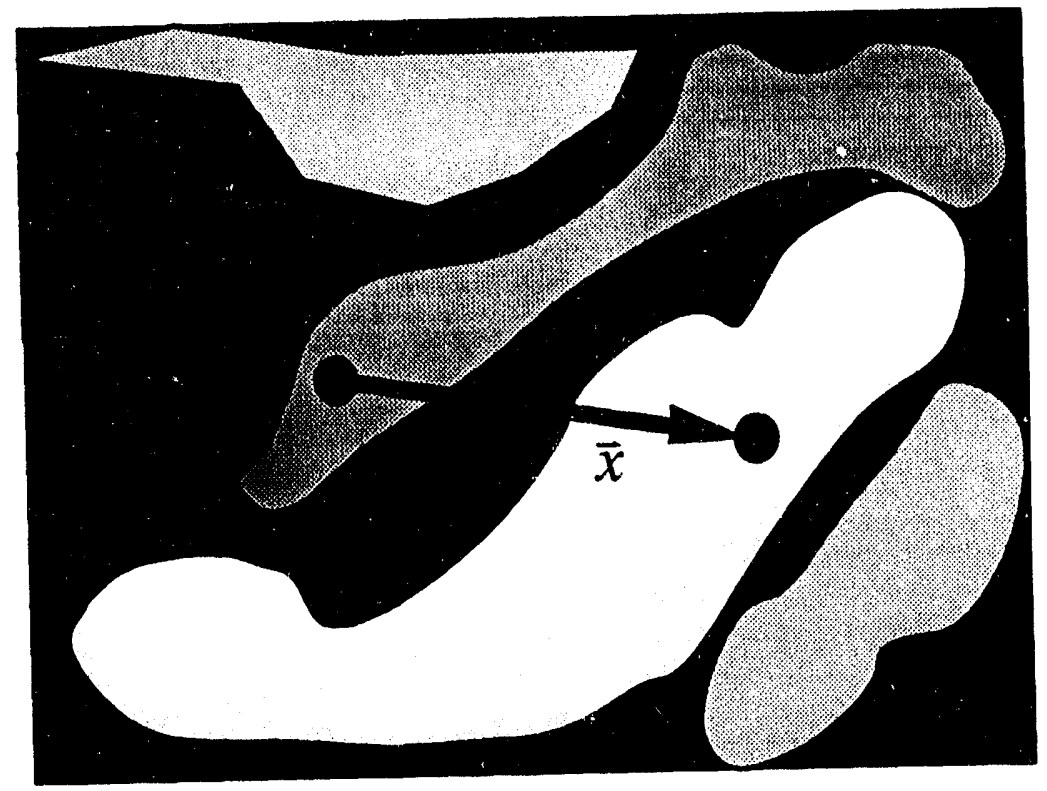

Figure 4.8. The pair correlation function that is determined by integrating $k \rightarrow \infty$ with the Porod law contains no atomic structure. A region is considered to be of a constant electron density with sharp boundaries. The pair correlation function (Eq. 3.1.7) for a vector $\bar{x}$ is thus the product of the electron density at the tail and arrow of vector $\vec{x}$, averaged over all starting points. 
been used to study oriented noncrystalline polymers, ${ }^{5}$ but to our knowledge have not been applied to vapor-deposited amorphous semiconductors or metals.

It should be stressed that direct Fourier transformation of the data is difficult due to truncation errors; the atomic scale structure (i.e. at large $k$ ) is not measured so that we cannot determine the 'true' CCF one would obtain by integrating over the entire reciprocal space. Despite this limitation, we have calculated the CCF by extending the observed intensities to $k \rightarrow \infty$ with the Porod law (as is most often done in SAXS studies $\left.{ }^{6}\right)$. The pair correlation function that is thus determined contains no information about the atomic structure. It assumes that the electron density for a particular region is a constant and that there are sharp boundaries between regions of different electron density (see figure 4.8).

\section{3.a Theory of the CCF}

As noted in Section 3.1, the pair correlation function is the average over all pair correlations of the deviation in effective electron density from the average. It is the Fourier transform of the observed $\mathrm{x}$-ray scattering function, the structure factor $S(\vec{k}, E)$. The CCF has been calculated for scans measured at one energy, $E=6912 \mathrm{eV}$, so for convenience the variable $E$ will be dropped from discussion although it is important to remember that $E$ defines an effective electron density. For cylindrically symmetric functions, $\gamma(\vec{x}, E)$ and $S(\vec{k}, E)$ can be expanded in terms of Legendre polynomials,

$$
\gamma\left(x, \theta_{x}\right)=\sum_{n=0,2,4 \ldots}^{\infty} P_{n}\left(\cos \theta_{x}\right) \gamma_{n}(x) \text { and } S\left(k, \theta_{k}\right)=\sum_{n=0,2,4 .}^{\infty} P_{n}\left(\cos \theta_{k}\right) S_{n}(k)
$$

where the coefficients $\gamma_{n}(x)$ and $S_{n}(k)$ satisfy the spherical Bessel transform 


$$
\gamma_{n}(x)=\frac{i^{n}}{2 \pi^{2}} \int_{0}^{\infty} k^{2} j_{n}(k x) S_{n}(k) d k
$$

and $\left(x, \theta_{x}, \phi_{x}\right)$ and $\left(k, \theta_{k}, \phi_{k}\right)$ are spherical polar coordinates in real and reciprocal space that describe $\gamma(\bar{x})$ (see figure 3.1). Due to the cylindrical symmetry, $\phi_{k}$ and $\phi_{x}$ do not enter into :ne final relations and are dropped from discussion. The $S_{n}(k)$ are readily obtained from the orthogonality relations, i.e.

$$
S_{n}(k)=(2 n+1) \int_{0}^{\pi / 2} S\left(k, \theta_{k}\right) P_{n}\left(\cos \theta_{k}\right) \sin \theta_{k} d \theta_{k}
$$

Only the even terms in $n$ are of interest, since there exists an assumed inversion symmetry with respect to the $x_{1}-x_{2}$ or $k_{1}-k_{2}$ plane. This need not be the case, however, as one can imagine a situation where correlations in the direction of film growth $+\hat{x}_{3}$ differ from those in the $-\hat{x}_{3}$ direction. 7

Legendre polynomials form a complete set, but it is not possible to uniquely determine all $S_{n}(k)$ from the finite data collected. For example, if there exist nine different radial scans in $k$ that span $\theta_{k}=0^{\circ}$ to $90^{\circ}$, the largest $n$ that can be determined is eight (the highest order polynomial one expects to fit nine data points is of order eight). Hence, large regions of reciprocal space must be sampled in order to approximate $S(k, \theta)$ as a Legendre series. Only those samples that lack long-range order and exhibit slight anisotropy are amenable to this method.

In contrast to atomic pair correlation functions used in studies of short-range order, the "independent scattering" of the individual particles has not been removed, since it does not appear obvious how to remove the self-scattering contribution from SAXS data. The observed scattering is from both particle and interparticle effects, and it 
is usually desirable to remove the particle scattering so that one can highlight the interparticle features. Indeed, for the case of large interparticle interference, it is still not possible to remove the contribution from the particles' self-correlations since there exists a distribution in particle size as well as particle interpenetration. This issue is discussed in more detail in Section 6.4. The CCF which is presented in this section thus includes the self-scattering contribution. The number of vectors that give an identical correlation between $x$ and $x+d x$ and $\theta_{x}$ and $\theta_{x}+d \theta_{x}$ is thus $4 \pi x^{2} \sin \theta_{x} \gamma\left(x, \theta_{x}\right) d \theta_{x} d x$, with a factor of 2 included for inversion symmetry.

\section{3.b Application of CCF to Amorphous Films}

Since the structure factor is very similar for all $a-\mathrm{Fe}_{\mathrm{x}} \mathrm{Ge}_{1-\mathrm{x}}$ samples $(\mathrm{x}<0.33)$--that is, there is a monotonic change in scattering intensity and shift in peak position with changes in $\theta_{k}$-we choose the 6.3 at.\% Fe sample as representative of the $x<0.33$ alloy and examine in close detail its CCF. It should be noted that each scan has been extrapolated in the normal way in order to perform the $k$ to $x$ transformation. Scans are extrapolated to large $k$ with the $\alpha=4$ power law and to $k=0$ with a Gaussian function, $a \exp \left[b\left(\theta_{\mathrm{k}}\right) k^{2}\right]$.

The anisotropic scattering data have been used to determine the first four $S_{n}(k)$ from Eq. 4.3.3 by employing a 7-point Simpson's rule. For each $k$, there are six measured points $\theta_{k}=90,75,60,45,30$, and $15^{\circ}$. The seventh is not measurable but not necessary to compute the $S_{n}(k)$, since the factor of $\sin \theta_{k}$ in the integrand forces the $\theta_{k}=0^{\circ}$ term of the total integrand to 0 . Figure 4.9a shows the computed $S_{n}(k)$. The $n=2$ and $n=4$ terms are far from negligible and illustrate the importance of including the anisotropy. The $n=6$ term, however, is much weaker, contributing far less to the series. The $S\left(k, \theta_{k}\right)$ computed from the $S_{n}(k)$ provides an approximation of better than $2 \%$ to the raw data, except near the SAXS maximum of the $\theta_{k}=90^{\circ}$ scan, where the greatest anisotropy is observed and 

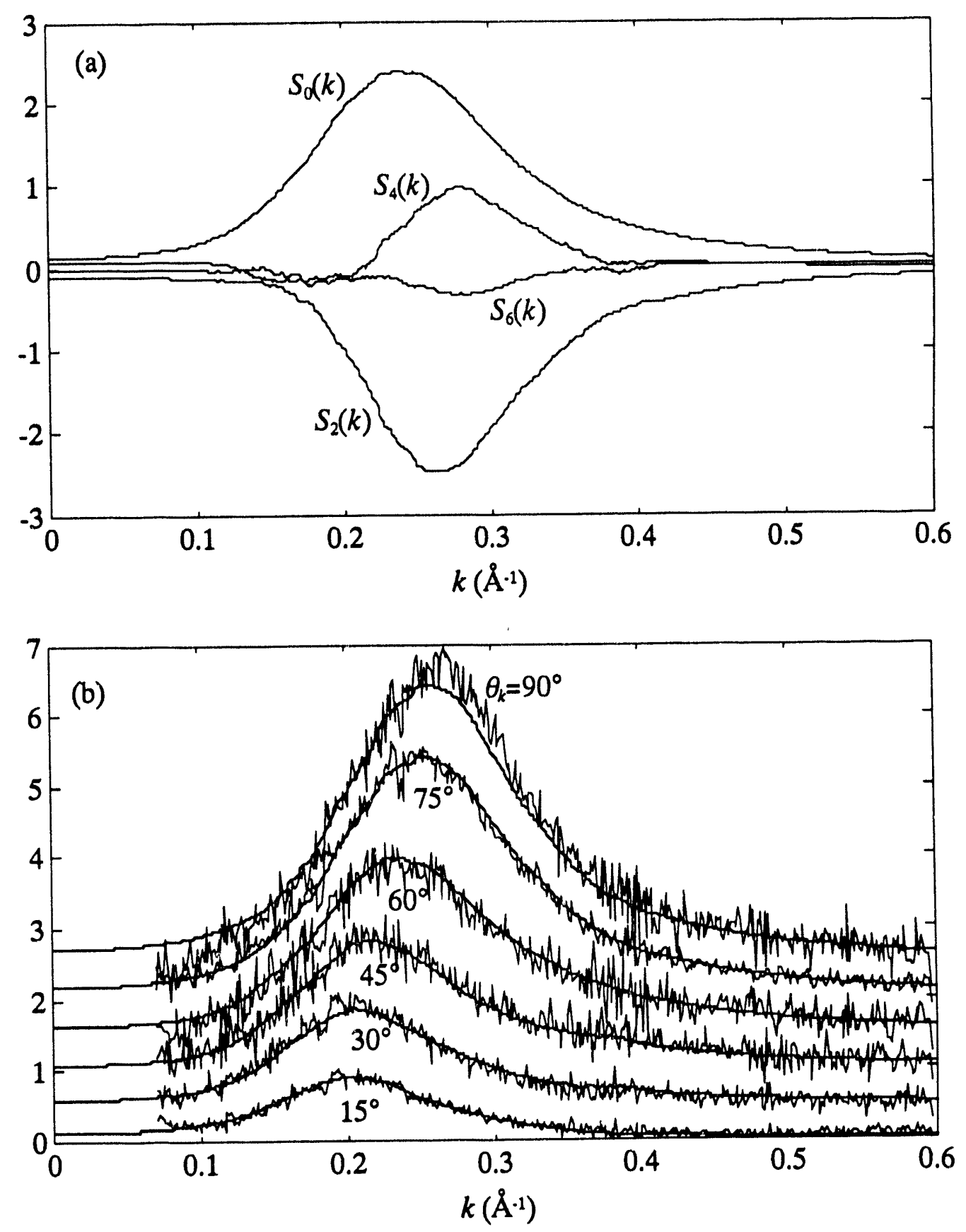

Figure 4.9. (a) Legendre cuefficients $r_{e}{ }^{2} S_{n}(k)\left(\mathrm{cm}^{-1}\right)$ for the 6.3 at. \% Fe sample. The $n=2$ and $n=4$ terms are far from negligible and illustrate the importance of including the anisotropy. The $n=6$ term, however, is much weaker, contributing far less to the series. (b) Comparison between the absolute scaled, unsmoothed data and the computed $S\left(k, \theta_{k}\right)$ from the $S_{n}(k)$. The Legendre sum provides an approximation of better than $2 \%$ to the data except near the SAXS maximum of the $\theta_{k}=90^{\circ}$ scan, where the greatest anisotropy is observed and the difference from that measured is $\sim 5 \%$. Plots offset for clarity. 
the fit is no worse than $5 \%$, a result of not including higher order $S_{n}(k)$ or, consequently, an inadequate sampling of reciprocal space (figure 4.9b). Such good agreement, then, helps validate the degree of $k$-space sampling and the 7-point Simpson's integration used in both the $\gamma(x, \theta)$ and $\left\langle\eta^{2}(E)\right\rangle$ calculations.

The spherical Bessel transforms of the $S_{n}(k)$ have been computed with a trapezoidal rule and the corresponding Legendre series summed to obtain the CCF (figure 4.10). The large maximum near $\vec{x}=0$ is from regions of electron density correlated with themselves--i.e. regions that are large enough to define a local electron density but small enough so that variations in density within are not observed. Correlations for $x<10 \AA$ also exhibit a large, positive $\gamma\left(x, \theta_{x}\right)$, due to the similarity of electron density of neighboring regions and are often considered the result of a "particle" of certain dimensions of a particular electron density. In this case, the dimensions of the particle appear elongated in the $x_{3}$-direction by more than a factor of 3 to 2 .

Beyond the contribution from self-correlation $(x>10 \AA)$, oscillations in $\gamma\left(x, \theta_{x}\right)$ about 0 are present. The CCF has been truncated at small $x$ in order to allow a close inspection of the oscillations. If $\gamma\left(x, \theta_{x}\right)<0$, then the difference in electron density from the mean for two points separated by $\bar{x}$ will be of opposite sign, on average. That is, one point will have an electron density greater than the mean electron density in the sample, and the second point, less than the mean. The amplitude of $\gamma\left(x, \theta_{x}\right)$ for this $\vec{x}$ is a measure of the likelihood that this is true any two given points in the sample separated by $\vec{x}$. The

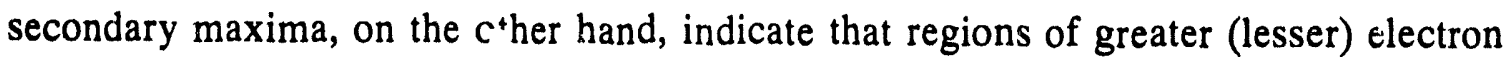
density than the mean exist and are correlated on a particular length scale. Similarly, the amplitude of the secondary maxima provide a measure of the likelihood that this is true for any two given points separated by $\vec{x}$. 
Table 4.1. Correlation parameters for different film directions $(6.3$ at. $\% \mathrm{Fe}$ ).

\begin{tabular}{|c|c|c|}
\hline$\theta_{x}\left({ }^{\circ}\right)$ & $\begin{array}{c}\text { FWHM } \\
\bar{x}=0(\AA)\end{array}$ & $\begin{array}{c}\text { secondary } \\
\text { maxima }(\AA)\end{array}$ \\
\hline $90\left(x_{1}-x_{2}\right)$ & 7.3 & $26.6,50.0,76.3$ \\
\hline 45 & 9.0 & $32.4,64.8$ \\
\hline $0\left(x_{3}\right)$ & 11.7 & 41 (v. weak) \\
\hline
\end{tabular}
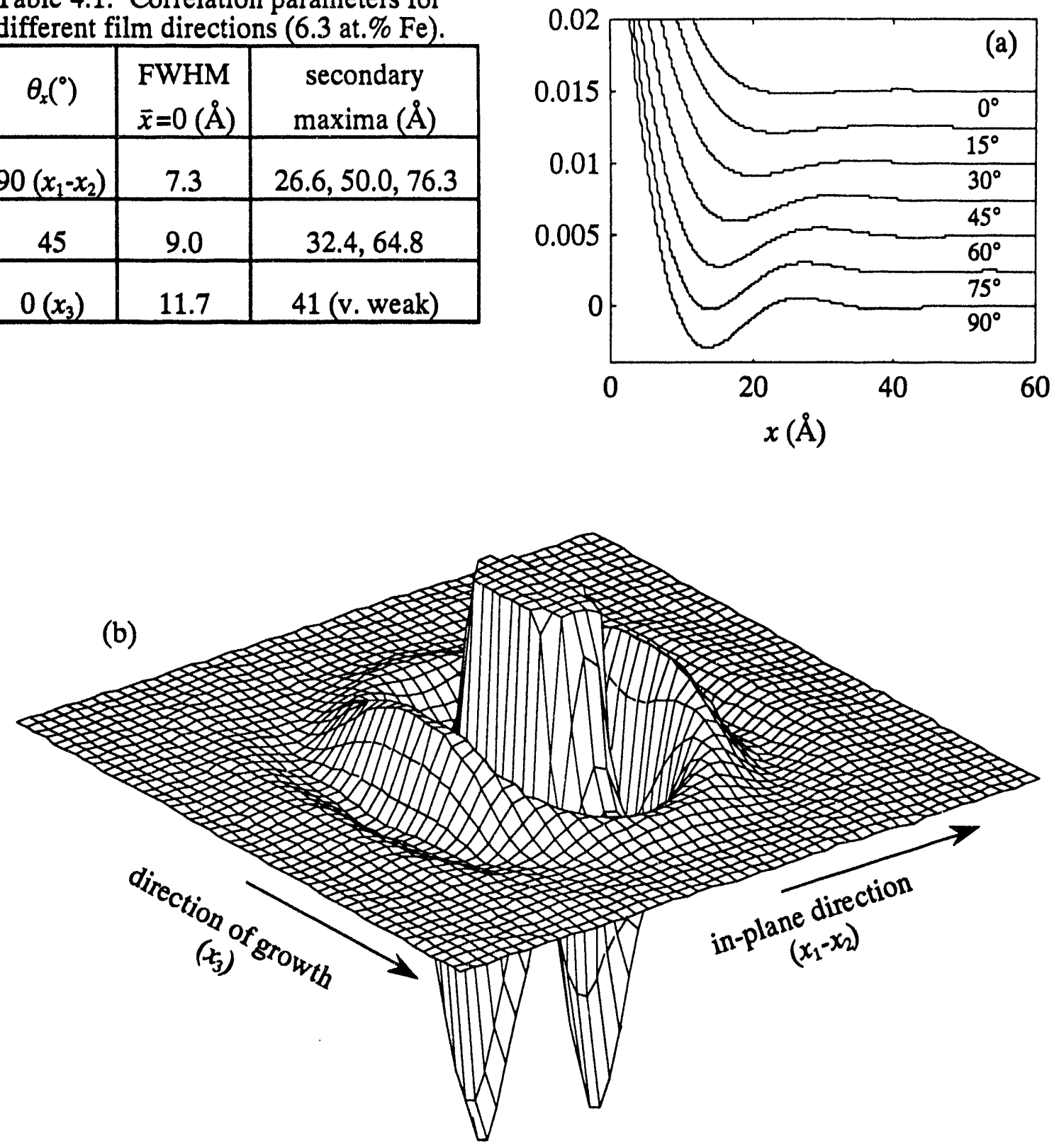

Figure 4.10. Cylindrical correlation function, $\gamma\left(x, \theta_{x}\right)$, in units of $\left(\mathrm{e} / \AA^{3}\right)^{2}$. (a) CCF along different radial directions. Plots offset and truncated at small $x$ in order to better observe the oscillations. (b) Three-dimensional mesh plot of the CCF. Mesh lines are spaced $3 \AA$ by $3 \AA ̊$. 
Table 4.1 shows the secondary maxima for a few $\theta_{x}$, which illustrate the strong oscillations in amplitude present for correlations in the plane of the sample. For those along $\theta_{x}=45^{\circ}$, the oscillations begin to wane in magnitude and frequency. Finally, for a correlation vector in the direction of film growth, oscillations are not obvious but exist at much greater distance than in the previous cases and at much smaller magnitude. In addition, table 4.1 indicates that the intraparticle correlations extend considerably further in $x_{3}$ than in $x_{1}$. These results are consistent with inhomogeneous and close-packed particle models which are developed in Chapter 6. By applying a cylindrical correlation function analysis, there exists a more direct and satisfying approach to the question of anisotropy, especially since the particle models assume monodispersity and an equilibrium liquid structure factor to explain interparticle interference (close-packed models) in the vapor-deposited state. 
References for Chapter 4

${ }^{1}$ M. Rice, Ph.D. Thesis, Stanford University (in preparation).

${ }^{2}$ M. Maret, J.P. Simon, B. Boucher, R. Tourbot, and O. Lyon, J. Phys.: Condens. Matter 4, 9709 (1992).

${ }^{3}$ M. Rice, S. Wakatsuki, and A. Bienenstock in Thin Film Structures and Phase Stability, ed. by B.M. Clemens and W.L. Johnson (Materials Research Society, Pittsburgh, PA, 1990), MRS Symp. Proc., Vol. 187, 53.

${ }^{4}$ N. Norman, Ph.D. Thesis, University of Oslo (1954).

${ }^{5}$ M.E. Milberg, J. Appl. Phys. 34, 722 (1962).

${ }^{6}$ Th. Gerber, private communication, 1993.

${ }^{7}$ L.C. Wilson, Ph.D. Thesis, Stanford University (1990). 


\section{Chapter 5}

\section{Amorphous $\mathrm{Mo}_{\mathrm{x}} \mathrm{Ge}_{1 \cdot \mathrm{x}}$ Results}

Since the amorphous $\mathrm{Fe}_{x} \mathrm{Ge}_{1 \cdot x}$ and $\mathrm{Mo}_{x} \mathrm{Ge}_{1 \cdot x}$ have fairly similar equilibrium phase diagrams for $x<0.33$, namely no solid solubility, one might expect the phase separation observed in $\mathrm{Fe}_{x} \mathrm{Ge}_{1 \cdot x}$ to be a good indicator as to what to expect for the $\mathrm{Mo}_{x} \mathrm{Ge}_{1 \cdot x}$ alloys. Indeed, phase separation is observed in $\mathrm{a}-\mathrm{Mo}_{\mathrm{x}} \mathrm{Ge}_{1 \cdot-\mathrm{x}}$, but the anisotropy is not as pronounced, and, more strikingly, phase separation disappears near $\sim 24$ at.\% Mo. Density calculations confirm endpoint compositions near 24-26 at.\% Mo for the samples that do exhibit phase separation, leading one to believe that the endpcint of phase separation is an intermetallic close in composition to an a-MoGe $e_{3}$. This is, of course, quite a bit different from the $\mathrm{Fe}_{\mathrm{x}} \mathrm{Ge}_{1 \cdot \mathrm{x}}$ results, where the endpoint composition is predicted close to an intermetallic like a- $\mathrm{FeGe}_{2}$.

\subsection{Anisotropy}

Figure 5.1 illustrates the structure factor at normal incidence for several $\mathrm{Mo}_{\mathbf{x}} \mathrm{Ge}_{1 \cdot x}$ $(0.065<x<0.236)$ alloys. The scattering is much weaker than that recorded from the Fe-Ge alloys. The data were collected at $E=10803 \mathrm{eV}$ with a $\mathrm{Xe} / \mathrm{CO}_{2}$ gas filled detector and have been background subtracted and placed on an absolute scale; the 12.4 at.\% Mo scan was 


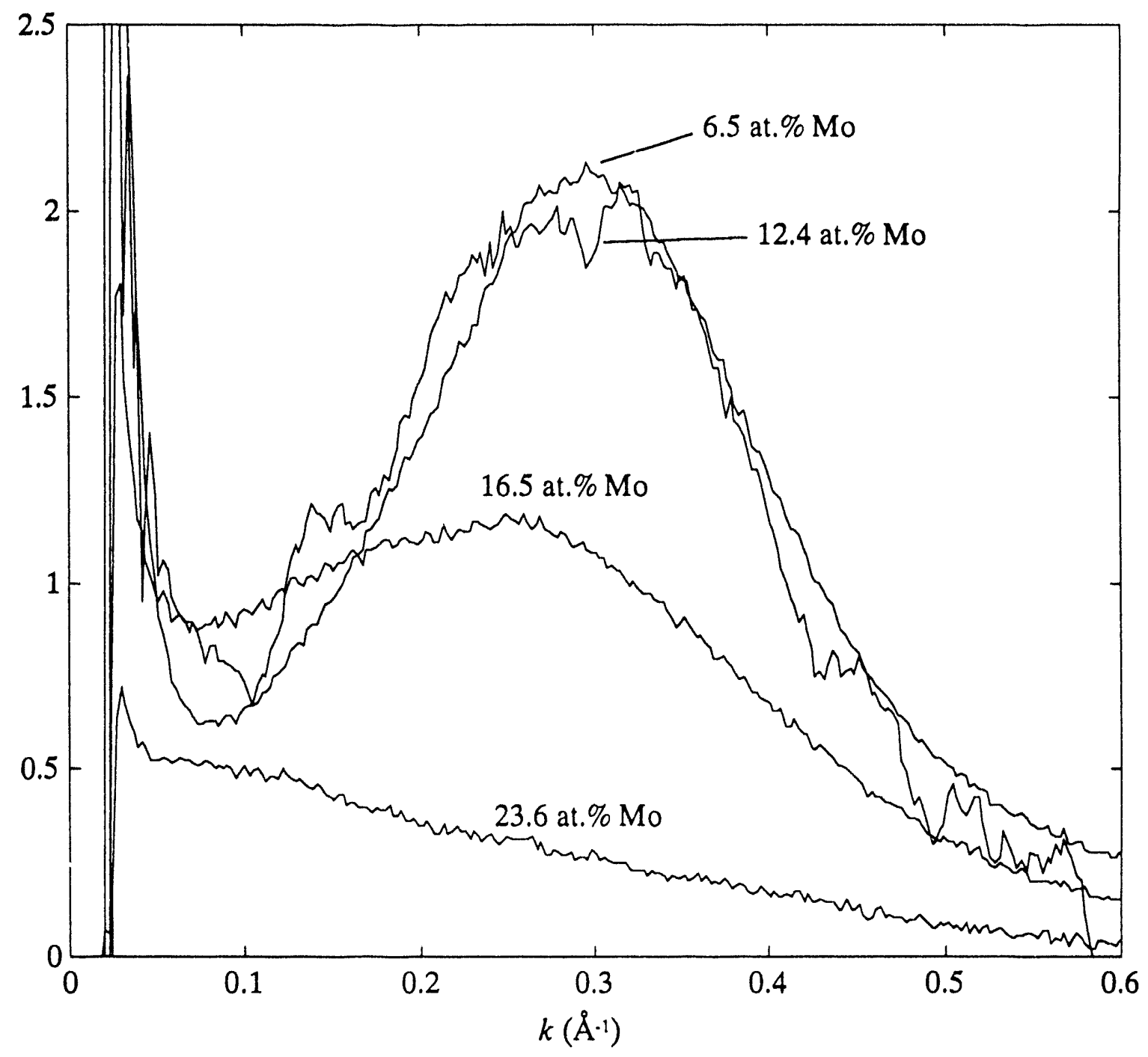

Figure 5.1. SAXS, $r_{e}^{2} S(k, 90)\left(\mathrm{cm}^{-1}\right)$, as a function of composition for the $\mathrm{Mo}_{\mathrm{x}} \mathrm{Ge}_{1 \cdot \mathrm{x}}$ alloys. Since the 12.4 at.\% Mo scan was collected with an $\mathrm{Ar} / \mathrm{CO}_{2}$ filled detector, it has a much lower signal-to-noise ratio than the others (collected with $\mathrm{Xe} / \mathrm{CO}_{2}$ gas). In the data presented above, the 12.4 at.\% Mo scan has been smoothed over an interval $\Delta k=0.02 \AA^{-1}$. $E=10803 \mathrm{eV}$. 
collected at a previous time with an $\mathrm{Ar} / \mathrm{CO}_{2}$ gas and has been smoothed over an interval $\Delta k=0.02 \AA^{-1}$. The 6.5 and 12.4 at.\% Mo samples exhibit the greatest magnitude of scattering, with it less intense for the 16.5 at.\% Mo sample, while little scattering is recorded for 23.6 at.\% Mo. The scattering that is recorded for this sample is extremely weak and is of a different kind, at small $k$, with only a small, if any, scattering maximum resolvable. The apparent maximum near $k \sim 0.1 \AA^{-1}$ may result, however, from an imperfect subtraction of the mica windows and/or from parasitic scattering from and around the main beam. Kortright and Bienenstock (cited previously, see Chapter 1) dit not observe appreciable SAXS of any kind for their 25 at.\% Mo sample (grown under identical conditions as the 23.6 at.\% Mo sample reported here) in the small-angle region spanning $0.01<k<0.15 \AA^{-1}$. It is thus likely that the SAXS reported for our sample is either from background sources or indicates that the volume fraction of the chemical inhomogeneity is decreasing rapidly and becomes essentially zero at -25 at.\% Mo.

This agrees with a previous structural study of Kortright and Bienenstock (see Chapter 1) who delineate three different structural regions of which the composition $\mathrm{x} \sim 0.23$ is at a boundary between two of the regions. As noted earlier, their SAXS study did not extend out to large enough $k$ to observe the nanoscale phase separation. Rather, with the observation of a weak, monotonically decreasing signal (localized below $k \sim 0.05$ $\AA^{-1}$ ) and a diffuse, constant intensity background, they concluded that the source of the scattering for $k<0.05 \AA^{-1}$ is unknown (likely cracks or voids) and that the diffuse signal, which shows a trend with composition, is related to a fine size scale inhomogeneity of $\sim 10 \AA$ that is a maximum at 8 at.\% Mo. The use of independent particle scatterers $(5 \AA$ spheres in this case) to model the diffuse background, however, is certainly not valid in a $k$-range where interparticle interference dominates (for example, see figure 6.10). The results do, however, provide an order of magnitude estimate of the size of inhomogeneity 
and are consistent with their other results (large-angle scattering, EXAFS) that are interpreted in terms of a model of nanoscale inhomogeneity for structural region $\mathrm{I}$.

The oblique transmission results for the 6.5 at.\% Mo sample at $E=7100 \mathrm{eV}$ are presented in figure 5.2. The anisotropy is not strong-the $\theta_{k}=0^{\circ}$ scan is only $\sim 1.5$ times that of the $\theta_{k}=60^{\circ}$ scan (in magnitude). The anisotropy in the $\mathrm{Fe}_{\mathrm{x}} \mathrm{Ge}_{1-\mathrm{x}}$ samples is more pronounced, with the $\theta_{k}=0^{\circ}$ scans $\sim 5$ times that of the $\theta_{k}=60^{\circ}$ scans, but the percent change in the position of the SAXS maximum with $\theta_{k}$ appear similar for both the Fe and Mo containing alloys. The positions of the maxima are, of course, highly dependent on the concentrations of the metal atoms, but, for similar compositions, the 6.5 at.\% Mo sample has $k_{\max } \sim 0.3 \AA^{-1}$ and for 6.3 at.\% $\mathrm{Fe}, k_{\max } \sim 0.275 \AA^{-1}$. Slightly longer length scales are thus expected in the Fe-Ge system, which can be more readily observed by comparing the CCFs.

Figure 5.3 shows the computed CCF for the 6.5 at.\% Mo sample, with views along the radial direction $x$ as a function of $\theta_{x}$ as well as a three-dimensional view of the full CCF. In comparison to the CCF from the 6.3 at.\% Fe sample (figure 4.9), the large, positive $\gamma\left(x, \theta_{x}\right)$ at small $x$ for this CCF does not extend to as large $x$ and the anisotropy of the FWHM of the peak at small $x$ is not as pronounced. That is, the "particles" are smaller, elongated by a factor $\sim 1.4$. The secondary maxima indicate that strong correlations exist between the particles in the plane of the sample, which persist with longer length scales as the correlation direction changes from an in-plane direction to that in the direction of growth. The CCF confirms that the overall length scale, from the size of particles to the oscillations in $\gamma\left(x, \theta_{x}\right)$, is shorter in the 6.5 at.\% Mo alloy than that for 6.3 at.\% Fe. Interestingly, the correlations in the direction of growth appear, relative to its in-plane correlations, stronger in the Mo-containing sample than in the corresponding Fe sample. 


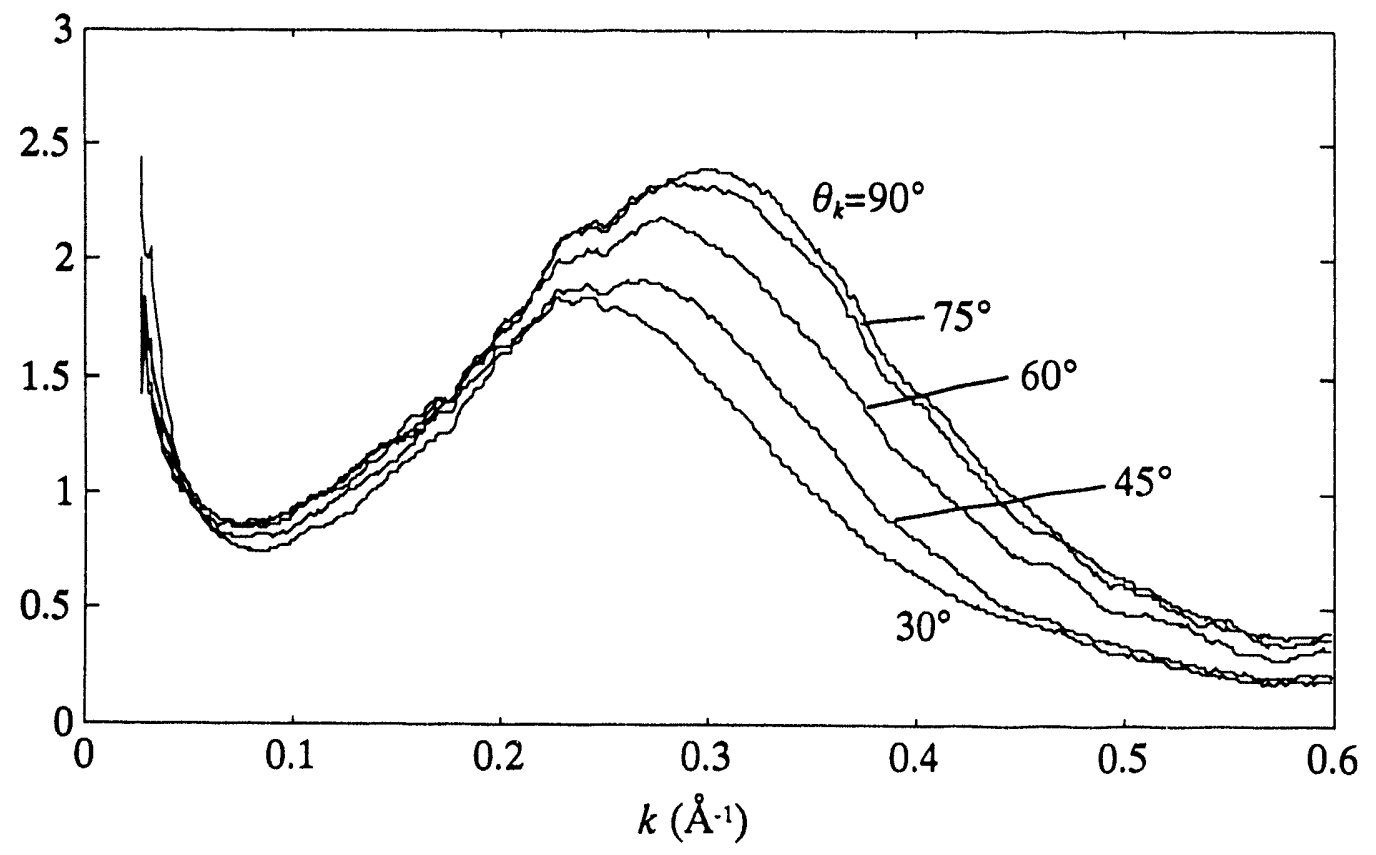

Figure 5.2 SAXS, $r_{e}{ }^{2} S\left(k, \theta_{k}\right)\left(\mathrm{cm}^{-1}\right)$, as a function of oblique transmission angle for the 6.5 at.\% Mo sample. Collected at $7100 \mathrm{eV}$. Data smoothed over an interval $\Delta k=0.02 \AA^{-1}$. 
Table 5.1. Correlation parameters for different film directions $\left(\mathrm{a}-\mathrm{MO}_{6.5} \mathrm{Ge}_{93.5}\right)$.

\begin{tabular}{|c|c|c|}
\hline$\theta_{x}\left({ }^{\circ}\right)$ & $\begin{array}{c}\text { FWHM } \\
x=0(\AA)\end{array}$ & $\begin{array}{c}\text { secondary } \\
\text { maxima }(\AA)\end{array}$ \\
\hline $90\left(x_{1}-x_{2}\right)$ & 6.2 & $22.5,53.3,61.0$ \\
\hline 45 & 7.0 & $25.5,50.8,67.8$ \\
\hline $0\left(x_{3}\right)$ & 8.6 & $29.1,49.3$ \\
\hline
\end{tabular}
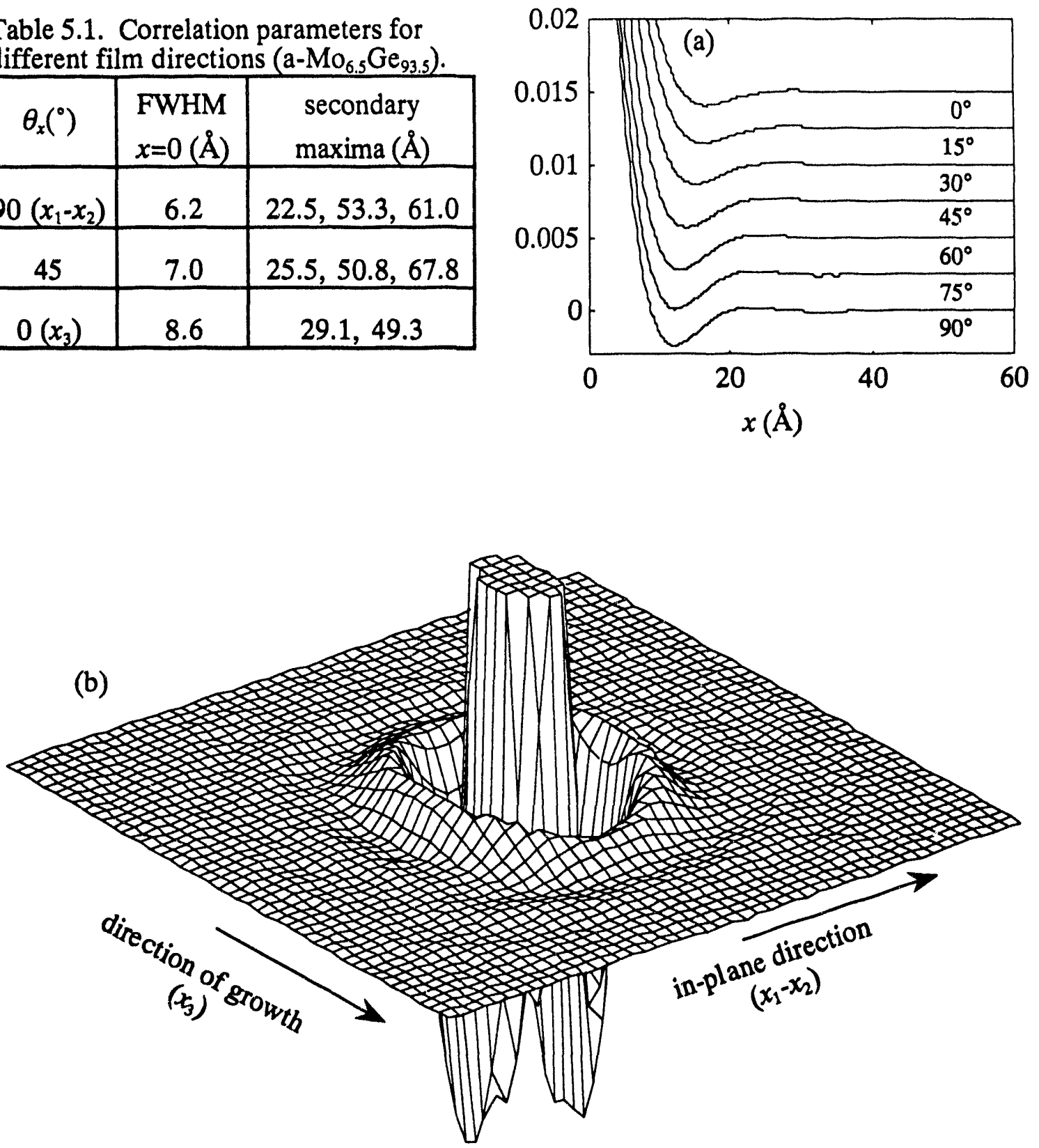

Figure 5.3. Cylindrical correlation function, $\gamma\left(x, \theta_{x}\right)$, in units of $\left(e / \AA^{3}\right)^{2}$. (a) CCF along different radial directions. Plots offset and truncated at small $x$ in order to better observe the oscillations. (b) Three-dimensional mesh plot of the CCF. Mesh lines are spaced $3 \AA$ by $3 \AA$. 


\subsection{DAS Results}

As mentioned previously, in the last period of data collection a $\mathrm{Xe} / \mathrm{CO}_{2}$ filled detector was used to improve the counting statistics at the Mo and Ge K-edges. By using a $\mathrm{Xe} / \mathrm{CO}_{2}$ counter gas and $\mathrm{Zn}$ filters to reduce Ge fluorescence, a coherent $\mathrm{x}$-ray scattering signal was observed at the Mo K-edge and anomalous scattering was thus possible. Figure 5.4 shows the anomalous scattering patterns for the 6.5 at.\% Mo alloy and the corresponding weighted-average PSFs (smoothed and unsmoothed). The relative change at the Mo edge is approximately a factor of 5-10 greater than that observed at the Ge edge. An $11 \%$ change in $f_{M O}(E)$ causes an approximate $30 \%$ change of the main scattering peak, whereas the $16 \%$ change in $f_{G e}(E)$ leads to a mere $6 \%$ intensity change. Although the weighted average PSFs have been obtained, the PSFs have not. The poor Mo edge SNR introduces large uncertainties in the PSFs whether using the standard Keating or Munro approaches. It is clear that the dominant change occurs at the Mo edge with a much smaller effect at the Ge-edge. Although Mo K-edge data were collected for only one sample (6.5 at.\% Mo) at normal transmission $\left(\theta_{k}=90^{\circ}\right)$, it is expected that the anomalous scattering results are similar for the 12.4 and 16.5 at.\% Mo alloys and at all oblique angles. The difficulty with the SNR and Ge fluorescence with limited beamtime allowed us a thorough study of only one of the strongest scattering samples at one transmission angle.

In a follow-up study, it would be interesting to study the 23.6 at.\% Mo sample with Mo and Ge K-edge measurements. After all, if there is still phase separation at this composition and the "particles" are fairly large, then there would be an appreciable separation of the particles because the a-Ge component would be a small fraction of the entire sample. As a result, there would be little interparticle interference and one would expect scattering of the type we observe. For this sample, then, the anomalous scattering could help discern the particle scattering from background or mica windows. 

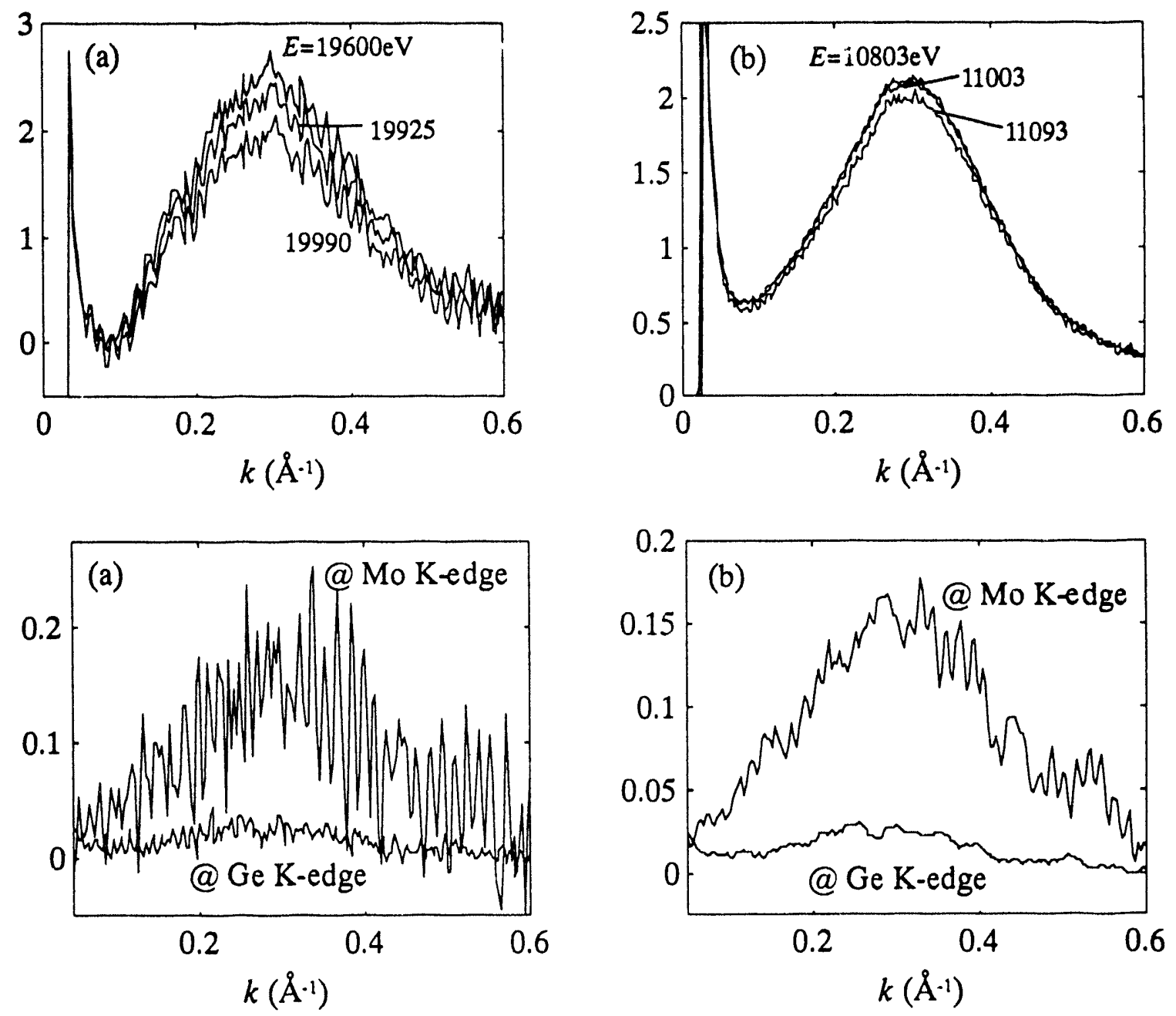

Figure 5.4. Anomalous scattering results at the (a) Mo and (b) Ge K-edges for 6.5 at.\% Mo sample. It should be stressed that 4-6 energies have been recorded at each edge to check for self-consistency, with only 3 shown for clarity in (a) and (b). (c) Weighted average PSFs. Absolute changes in SAXS at the Mo and Ge K-edges, scaled by the relative change in $f^{\prime}(E)$ at each edge and $r_{e}{ }^{2}$. At the Ge edge, $E_{1}=-300$ and $E_{2}=-10 \mathrm{eV}$ below the edge, and $E_{1}=-400$ and $E_{2}=-10 \mathrm{eV}$ below the Mo edge. (d) Data smoothed over an interval $\Delta k=0.02 \AA^{-1}$ with the difference at the Ge edge a factor of $-5-10$ less than that at the Mo edge. 


\subsection{Mean-Square Fluctuation in Density}

Since only the $\theta_{k}=90^{\circ}$ scans could be recorded with the $\mathrm{Xe} / \mathrm{CO}_{2}$ detector gas at the Mo K-edge, the mean-square fluctuation in effective electron density cannot be determined as a function of Mo K-edge energies. In an earlier run, the oblique transmission measurements were performed as a function of energy at the Ge K-edge, but the change in scattering with energy is so small that no resolvable change in $\left\langle\eta^{2}(E)\right\rangle$ can be observed. This latter result is expected, since the number density of Ge atoms is essentially constant throughout the sample.

At a single, lower energy $(7100 \mathrm{eV})$, which allows for an improved SNR, the oblique measurements were performed on the 6.5 and 12.4 at.\% Mo samples. The calculated mean-square fluctuation in density per Ge number density are listed in table 5.2 for the two different power-law extrapolations ( $\alpha=4$ and 5 ). Since the mean-square fluctuation in density is known only at one energy, the slope method to determine the endpoint composition discussed in Section 4.2 cannot be used. As with any SAXS study, however, the endpoint compositicn can still be determined by dividing out the atomic scattering factors. For a model of a sharp transition between the two phases, Eq. 4.2.6 is written as

$$
\frac{x}{1-x}\left(n_{M o}^{q}-\frac{x}{1-x} n_{G e}\right)=\left[\frac{\left\langle\eta^{2}\right\rangle}{n_{G e}}\right] \frac{1}{\left|f_{M o}\right|^{2}}
$$

for a x-ray energy of $7100 \mathrm{eV}$ (and similarly for Eq. 4.2 .10 and the $50 \%$ interface width model). Then as in Section 4.2, for a given $n_{G e}$, a $n_{M o}^{q}$ can be determined and hence the composition $q$ of phase $\mathrm{Mo}_{q} \mathrm{Ge}_{1 \cdot q}$ (Eq. 4.2.7).

When interpreted in terms of sharp and 50\% diffuse transition widths between the two phases, the computed endpoints $q$ range from $\sim 23-27$ at. $\%$ Mo, summarized in table 
5.2. This is in remarkable agreement with the disappearance of the SAXS at 23.6 to 25 at.\% Mo and is consistent with the structural model of Kortright and Bienenstock, who argue that structural regions I and II meet at approximately 23 at.\% Mo. These results indicate that the phase separation likely results in a film with regions of a-Ge and a Ge-rich intermetallic, close in stoichiometry to an amorphous $\mathrm{MoGe}_{3}$. Interestingly, c- $\mathrm{MoGe}_{3}$ is not in the equilibrium phase diagram; alloys with this composition are phase-separated into c-Ge anu c-MoGe ${ }_{2}$. This, of course, does not rule out atomic arrangements in the asdeposited alloys that prefer a short-range order that results in an overall composition of $\mathrm{MoGe}_{3}$.

Table 5.2. Results from the deinsity and endpoint composition calculations for $E=7100 \mathrm{eV}$.

\begin{tabular}{|c|c|c|c|c|c|c|}
\hline \multirow{2}{*}{$\begin{array}{c}\text { Sample } \\
\text { (at.\% Mo) }\end{array}$} & \multicolumn{3}{|c|}{ Sharp Boundaries $(\alpha=4)$} & \multicolumn{3}{|c|}{$50 \%$ Diffuse $(\alpha=5)$} \\
\hline & $\begin{array}{r}\left\langle\eta^{2}\right\rangle / n_{G e} \\
\left(e^{2} / \AA^{3)}\right. \\
\end{array}$ & $\begin{array}{c}q \cdot 100 \\
n_{G e}=a-G e\end{array}$ & $\begin{array}{c}q \cdot 100 \\
n_{G e}=c-G e\end{array}$ & $\begin{array}{c}\left\langle\eta^{2}\right\rangle / n_{G e} \\
\left(e^{2} / \AA^{3)}\right. \\
\end{array}$ & $\begin{array}{c}q \cdot 100 \\
n_{G e}=\mathrm{a}-\mathrm{Ge}\end{array}$ & $\begin{array}{c}q \cdot 100 \\
n_{G e}=c-G e\end{array}$ \\
\hline 6.5 & 1.539 & 27.3 & 26.5 & 0.9893 & 24.1 & 23.5 \\
\hline 12.4 & 1.846 & 24.3 & 23.9 & 1.187 & 23.6 & 23.2 \\
\hline
\end{tabular}




\section{Chapter 6}

\section{Models of Phase Separation}

The $\mathrm{x}$-ray scattering results lead naturally to the following two questions: does a unique model structure exist that incorporates this anisotropy and are there growth models that explain how a vapor-deposited film evolves into such a structure? X-ray scattering cannot unambiguously identify a particular model or structure, but of course any model must be able to reproduce all of the scattering results. This chapter is devoted to trying to answer these two questions and arriving at a plausible model.

As an attempt to explain these data, it has been suggested that the surface plays an important role. Adatoms have a few seconds to rearrange on the surface before being covered by the advanced surface and governed by the much slower bulk transport coefficients. With mobilities greater and activation energies lower at the surface than in the bulk, it seems likely that most of the structural properties are determined at the surface, with only slight modification in the bulk (e.g. structural relaxation). Our efforts to model the data initially focused on the growth process itself, but simple growth models that we have considered were inconsistent with the data. This chapter begins by discussing these simple models and then proceeds to a discussion of particle models that have been constructed to fit the features of the observed scattering. 
$\bar{k}=\left(k_{12}, k_{3}\right)$. For a film that evolves as it grows, the new surface can be considered related to the previous layer by a simple recursion relation:

$$
\tilde{\eta}\left(k_{12}, x_{3}+\Delta\right)=M\left(k_{12}, \Delta\right) \tilde{\eta}\left(k_{12}, x_{3}\right)
$$

with $M\left(k_{12}, \Delta\right)$ a replication factor that transforms the previous layer to the next. This can be transformed to an equivalent first-order linear differential equation

$$
\frac{\partial \tilde{\eta}\left(k_{12}, x_{3}\right)}{\partial x_{3}}=R\left(k_{12}\right) \tilde{\eta}\left(k_{12}, x_{3}\right)
$$

where $R\left(k_{12}\right)$ is an amplification factor that specifies those Fourier components in electron density that are stable and grow exponentially $\left(R\left(k_{12}\right)>0\right)$. The film consists of interconnected waves of electron density, and as the film grows thicker, select waves dominate. This equation is that suggested by Srolovitz and Kessler as a modification of Cahn's linear theory ${ }^{2}$ of spinodal decomposition for phase separation during growth. They show that it is straightforward to include a moving reference frame into the equations, which modifies Cahn's amplification factor $R\left(k_{12}\right)$ by a constant, remaining a fourth-order polynomial. For a given depth $x_{3}$ in the sample (corresponding to different times during the deposition process), the solution of Eq. 6.1.5 is

$$
\bar{\eta}\left(k_{12}, x_{3}\right)=\bar{\eta}\left(k_{12}, 0\right) e^{R\left(k_{12}\right) x_{3}},
$$

where $\bar{\eta}\left(k_{12}, 0\right)$ is the initial density distribution at the film's substrate.

This is the traditional Cahn theory of spinodal decomposition but with the time variable replaced by the $\hat{x}_{3}$ direction of growth. Some mention should be made of the 
that for some choices of thickness, the high- $k$ ripples cause the scattering peak to shift inward with decreasing $\theta_{k}$. In these cases, however, the amplitude change is not in agreement with data.

As another specific example, consider a film that grows layer by layer with no appreciable interaction between layers--this is exactly opposite to the columnar model. An approximation to the film's structure is then to consider the phase uncorrelated in the $x_{3^{-}}$ direction. The average over all pair correlations that are not in the same layer is 0 :

$$
\gamma(\bar{x})=\left\langle\eta\left(\bar{x}_{0}\right) \eta\left(\vec{x}_{0}+\bar{x}\right)\right\rangle_{\bar{x}_{0}} \cdot \delta\left(x_{3}\right) .
$$

The structure factor for such a model is simply $S\left(k, \theta_{k}\right)=S\left(k \sin \theta_{k}, 90^{\circ}\right)$. The model spectra are illustrated in figure $6.1 \mathrm{~d}$ and also show no agreement with experiment. There is no intensity change with tilt and the peak shift is incorrect.

\section{1.b Dynamic Structure: Surface Evolution}

Another approach is to assume that the surface structure is not static during the deposition process--the film structurally evolves as it grows. A layer deposited on the surface adapts to the morphology it "sees" from the previous layer and adjusts even further for a limited time until covered by the advanced surface. The completed film, then, consists of layers stacked one on top of the other, each depending on the previous layer. A simple linear first-order differential equation can be developed to study phase separation in growing films (Srolovitz and Kessler ${ }^{1}$ ). For a given depth $x_{3}$ in the film (with $x_{3}=0$ the film/substrate interface), let the two-dimensional Fourier transform in $x_{1}$ and $x_{2}$ be given by $\bar{\eta}\left(k_{12}, x_{3}\right)$, where an isotropy is assumed in the $x_{1}-x_{2}$ plane so the reciprocal vector is 


\subsection{Simple Two-Dimensional Growth Picture}

The plan is to show that the two-dimensional models considered fail to explain the strong anisotropy in the amorphous films. This will be done with some general arguments, and then a few simple models are constructed to illustrate this point. They explicitly include correlations due to chemical modulations, thermal fluctuations, etc., in the plane of the film and, depending on the model, correlations in the direction of growth $x_{3}$. The full SAXS spectrum is computed for each model and results presented in terms of the radial component $k$ and polar angle $\theta_{k}$ of the total scattering vector $\vec{k}$.

\section{1.a Static Structures}

One specific structure common to vapor-deposited films is that from a columnar growth process. For a columnar structure, perfect phase correlation exists in the direction of growth--the electron density at $\bar{x}$ is the same as the density at point $\vec{x}=\left(x_{1}, x_{2}, 0\right)$. To compute the structure factor, we have from Eq. 3.1.1:

$$
S\left(\vec{k}_{12}, k_{3}\right)=\frac{1}{V}\left|\int_{0}^{T} e^{i k_{2} x_{3}} d x_{3}\right|^{2}\left|\int_{A} \rho\left(\vec{x}_{12}\right) e^{i \vec{k}_{12} \bar{x}_{12}} d^{2} x_{12}\right|^{2},
$$

with a finite film thickness $T$. The integral can be evaluated and the solution expressed in terms of polar coordinates and the structure factor at normal incidence:

$$
S\left(k, \theta_{k}\right)=S\left(k \sin \theta_{k}, 90^{\circ}\right) \frac{\sin ^{2}\left(k T \cos \theta_{k} / 2\right)}{\left(k T \cos \theta_{k} / 2\right)^{2}}
$$

where an in-plane isotropy has been assumed. Given a similar SAXS spectrum as for the amorphous films at normal incidence $\left(\theta_{k}=90^{\circ}\right)$, the peak shifts outward and decreases markedly in intensity with decreasing $\theta_{k}$ (figure 6.1a-c). Figure 6.1(c) shows, however, 


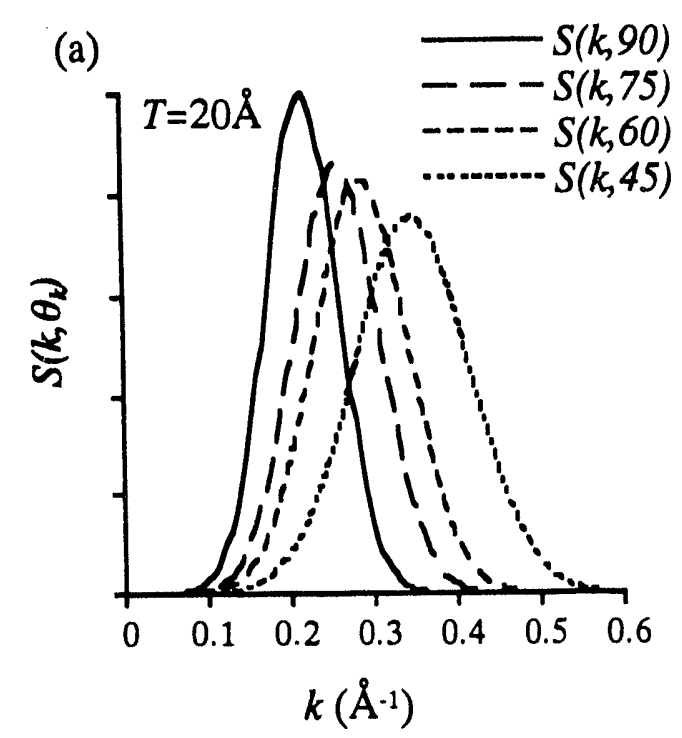

(c)

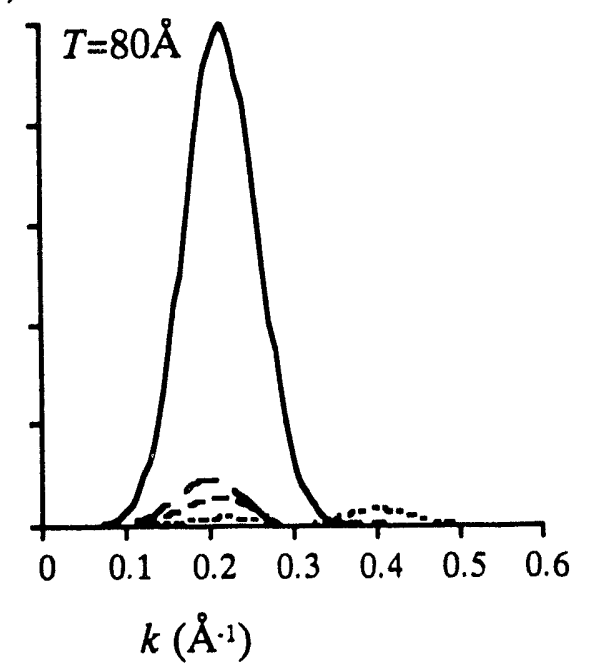

(b)

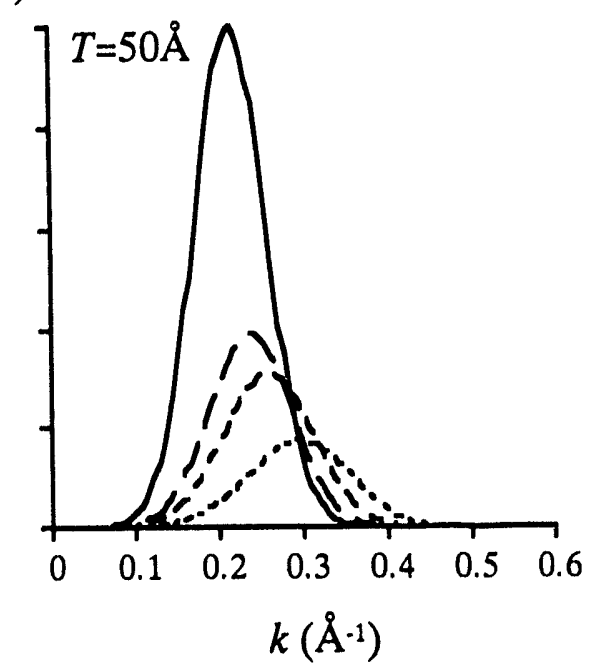

(d)

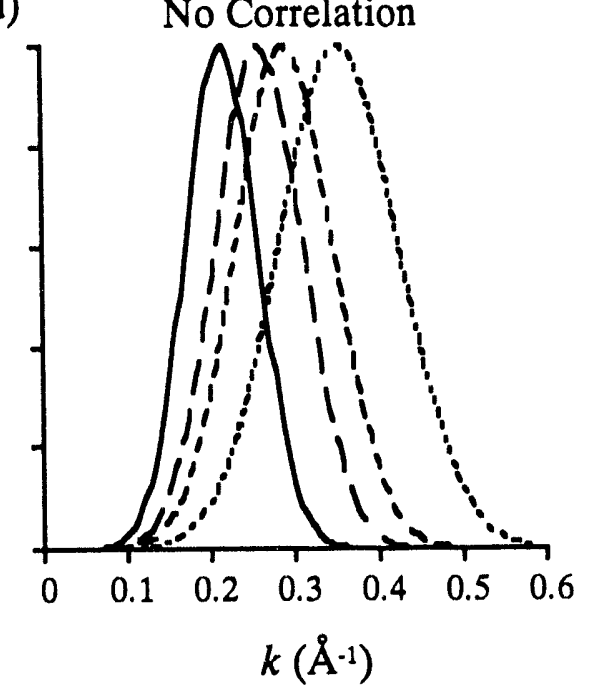

Figure 6.1. $S\left(k, \theta_{k}\right)$ for the columnar model (a-c) for various column heights $T$ and for the model of no correlation in the direction of growth (d). Neither model can reproduce the observed results from the oblique transmission experiment. 
assumptions in the theory. (a) The film is deposited at a rate $v$, with surface diffusion assumed to occur to a depth $\Delta$ interatomic spacing and with bulk diffusion assumed negligible. In-plane isotropy is assumed as well; that is, diffusion on the surface is considered equally probable in any direction. (b) The equations are linearized and hence good only during the initial stages of decomposition (early-time or early-growth approximation). Since the non-linear terms in the diffusion equation have been discarded, the theory is only valid for a short time after which other processes (nucleation, coarsening, etc.) have a greater chance of occurring. (c) Fluctuations during the growth process are neglected.

The structure factor for a film of thickness $T$ is

$$
S\left(k_{12}, k_{3}\right) \propto\left\langle\left.\eta\left(k_{12}, 0\right)\right|^{2}\right\rangle\left[\frac{e^{2 R\left(k_{12}\right) T}-2 e^{R\left(k_{12}\right) T} \cos \left(k_{3} T\right)+1}{R\left(k_{12}\right)^{2}+k_{3}^{2}}\right] .
$$

Figure 6.2 shows the resulting structure factor for a certain amplification factor $R(k)$, given in the Srolovitz-Kessler theory as $R\left(k_{12}\right)=\left(R\left(k_{12}\right)_{\mathrm{Cahn}}-v / \Delta\right) / v$, with $R\left(k_{12}\right)_{\text {Cabn }}$ the traditional Cahn amplification factor, $v$ the film deposition rate, and $\Delta$ the thickness per layer of deposited material. The averaged $\left|\eta\left(k_{12}, 0\right)\right|^{2}$ is an assumed Ornstein-Zernike relation of thermal fluctuations above the critical point. Since most amplification factors in the theory are quite similar, it appears almost certain that this model of surface generated spinodal decomposition cannot explain the composition modulations in the films. In addition, since this model is valid only during the initial stages of phase separation, it cannot explain a structural evolution that evolves throughout the entire thickness of our films, since they are quite thick (microns). It does, however, provide a picture of how the phase separation might initially start and predict a columnar type structure. 

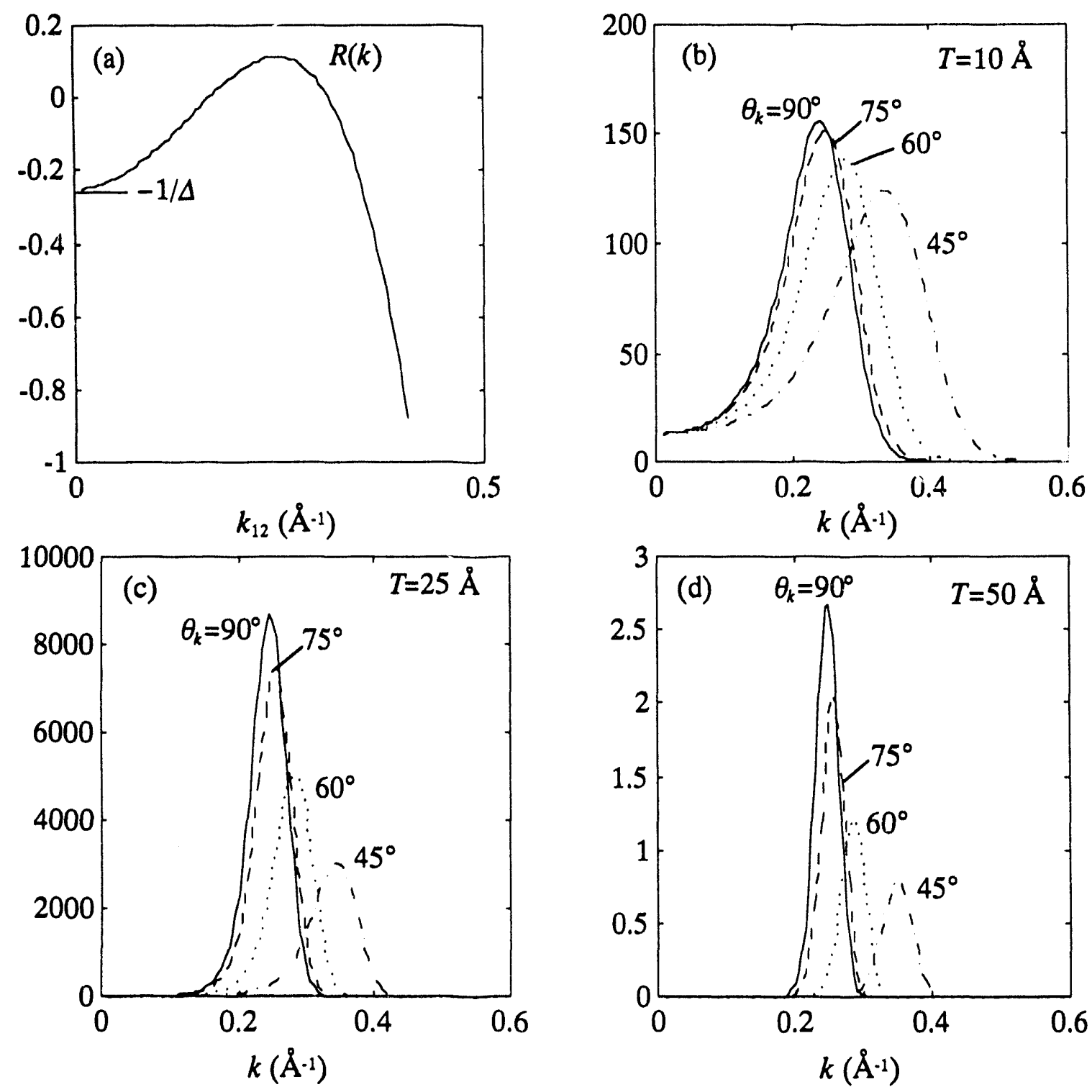

Figure 6.2. $S\left(k, \theta_{k}\right)$ for the surface evolution model. (a) Amplification factor; (b) the computed $S\left(k, \theta_{k}\right)$ with an Ornstein-Zernike prefactor, amplification factor given in (a), and film thickness $T=10 \AA$; (c) $T=25 \AA$; (d) $T=50 \AA$. 
If a spinodal decomposition model is to be applied, there needs to be a mechanism for breaking up the columnar coherence. Eq. 6.1.5 does not permit new fluctuations to intermittently unseat the steady exponential progression of the phase separation. The data suggest that non-trivial correlations exist in the direction of growth, and they cannot be modeled by these simple approaches. In fact, as will be demonstrated in the next two sections, a critical feature lacking in these models is the ability to nucleate new layers with Fourier coefficients of a completely new amplitude and, most importantly, phase.

\subsection{Single Particle Models}

Whether phase separation occurs via a spinodal decomposition or nucleation process, the late-time structures are fairly similar: there exist regions of well-defined electron density. Regions of well-defined electron density are believed to exist in these alloys since the large- $k$ side of the scattering pattern exhibits a power law close to the $1 / k^{4}$ Porod law of smooth interfaces. This is a part of reciprocal space where interparticle interference effects are less important while scattering from surfaces of the regions in space and electron density dominate. Consequently, one approach is to consider the scattering due to distinct particles of electron density, $\rho(\vec{x})$, embedded in a constant "background" density. To describe the small-angle scattering results from a collection of particles, we need to understand not only the contribution of the single particle to the scattering pattern but also the interparticle interference effects that modify the spectrum. To motivate this discussion, we first start by separating the particle from the interparticle scattering and then proceed to a study of the single particle models and interparticle interference.

For a collection of $N$ particles each of volume $V_{m}$ in a total volume $V$, the Fourier transform over the electron density can be written as a sum over the transform of each particle, 


$$
\int_{V} v(\vec{x}) e^{-i \vec{k} \cdot x} d^{3} x-\sum_{m} e^{-i k \cdot \bar{x}_{m}} \int_{V_{m}} v_{m}(\vec{x}) e^{-i \vec{k} \cdot x} d^{3} x
$$

with $v_{m}(\vec{x})$ the difference in electron density of particle $m$ from the background as a function of $\bar{x}$ within the particle with respect to the coordinates of particle $m, \vec{X}_{m} . v(\vec{x})$ is similar to $\eta(\bar{x})$ in Eq. 3.1.4, except it is the deviation in electron density of the particle from the background and not from the average. As with the atomic form factor, denote $F_{m}(\bar{k})$ as the particle $m$ "form factor" and equal to the integral on the left-hand side of Eq. 6.2.1. To compute the structure factor, we use similar arguments as in the derivation of Eq. 3.1.5:

$$
S(\bar{k})=\frac{1}{V}\left|\sum e_{m}^{-i k \cdot \bar{x}_{m}} F_{m}(\bar{k})\right|^{2} .
$$

Since the observed structure factor is that from the assembly of particles averaged with equal probability over all orientations in space and sizes of particles consistent with the symmetry of the system, Eq. 6.2 .2 thus becomes:

$$
S(\bar{k})=\rho_{p}\left\langle|F(\bar{k})|^{2}\right\rangle+\frac{1}{V}\left\langle\sum_{m} \sum_{n=m} e^{-i \bar{k} \cdot\left(\bar{x}_{m}-\bar{x}_{n}\right)} F_{i}(\bar{k}) F_{j}^{\cdot}(\bar{k})\right\rangle,
$$

with $\langle\ldots\rangle$ the averaging operator, $\rho_{p}$ the density of particles, and the $m=n$ term explicitly calculated. The first term is the scattering from individual particles, and the second is the contribution from interparticle interference effects. Although first derived by Debye in 1930 for atoms (the $F_{i}(\bar{k})$ known as the atomic scattering factors), the equations can be analogously applied to particle scattering. It should be noted that nothing has been assumed about the particles at this point--they are polydisperse, inhomogeneous, etc. For 
the remainder of this section, single particle models are presented as an attempt to explain the anisotropic structure factors.

\section{2.a Homogeneous Single Particles}

If a collection of particles is sufficiently dilute, then a common practice is to neglect interparticle interference effects. Then, the structure factor originates from individual particles, and the first term in Eq. 6.2.3 dominates the second. However, the problem is intractable without some simplifying assumptions. For a homogeneous particle (i.e. $v(\vec{x})=v$, a constant), the form factor for an ellipsoid is:

$$
F(\bar{k})=\int_{v_{e}} v(\bar{x}) e^{-i k \cdot x} d^{3} x=v \int_{v_{0}} e^{-i k \cdot q} d^{3} x
$$

with $V_{c}$ the volume of the ellipsoid with axes $\left(R_{1}, R_{2}, R_{3}\right)$. An easy way to perform the integral over the ellipsoid is to transform the integration to one for a sphere. ${ }^{3}$ Let $x_{1}{ }^{\prime}=x_{1} / R_{l}, x_{2}{ }^{\prime}=x_{2}{ }^{\prime} R_{2}$, and $x_{3}{ }^{\prime}=x_{3} / R_{3}$, then Eq. 6.2.4 simplifies to:

$$
F(\bar{k})=v R_{1} R_{2} R_{3} \int_{\substack{\text { untit } \\ \text { sphere }}} e^{-i\left(k_{1} R_{1}, k_{2}, R_{2}, k R_{3}\right) \cdot x^{\prime}} d^{3} x^{\prime}=v \Phi\left(k R_{e}\right) V_{e},
$$

with $\Phi(u)=3 \frac{\sin u-y \cos u}{u^{3}}, V_{e}=4 / 3 \pi R_{1} R_{2} R_{3}$, and $k R_{e}$ given by $\sqrt{k_{1}^{2} R_{1}^{2}+k_{2}^{2} R_{2}^{2}+k_{3}^{2} R_{3}^{2}}$. The observed structure factor for a dilute solution is thus determined by averaging Eq. 6.2.5 over all sizes and equivalent orientations of ellipsoids.

If the particles can be treated as identical (i.e. a monodisperse solution) then the averaging involves only that of particle orientation. In most cases, the particle orientation is averaged over all directions, so the resulting structure factor is isotropic. It is possible, however, to average over all ellipsoids oriented along a particular direction. Cargill ${ }^{4}$ 


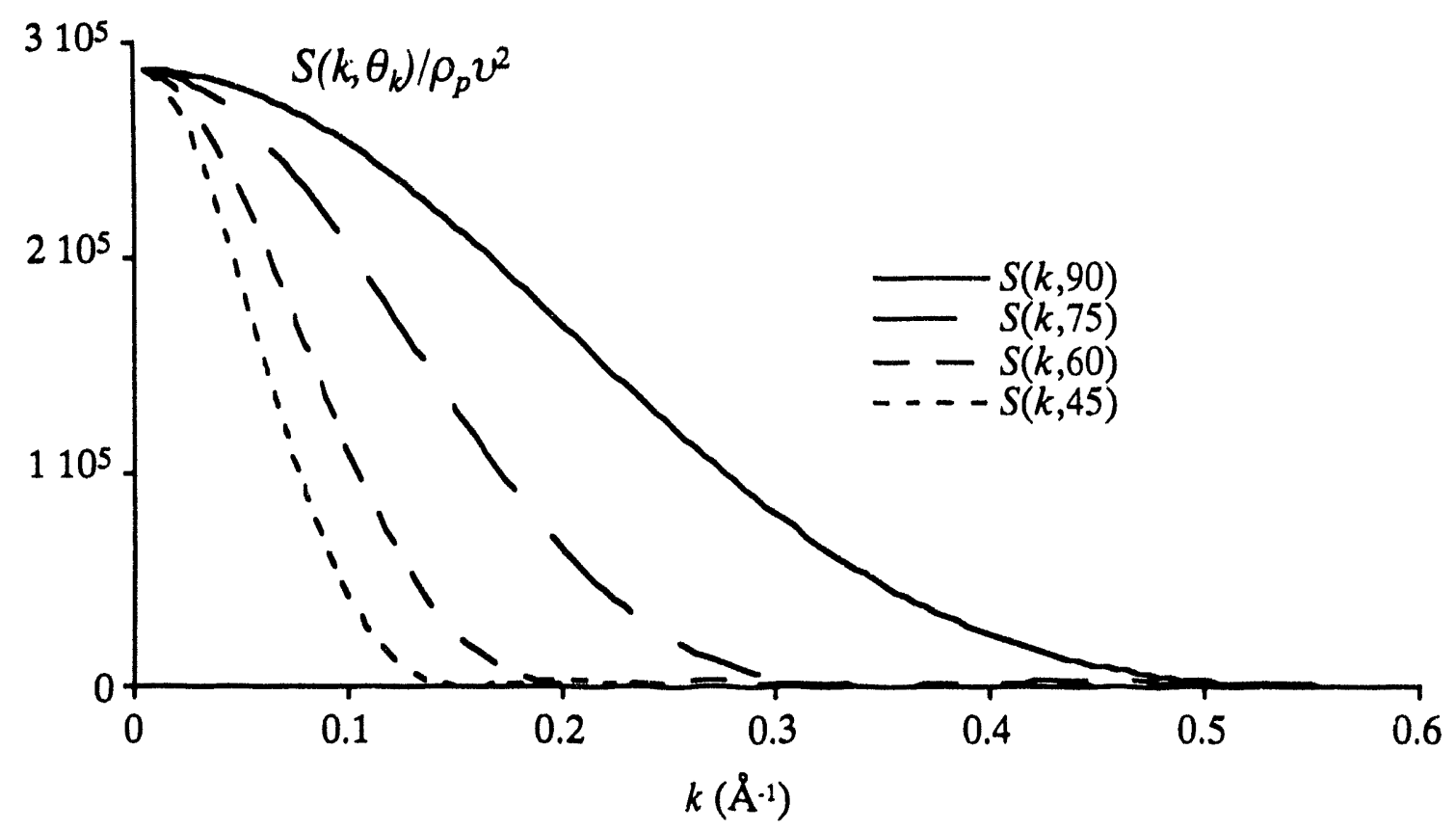

Figure 6.3. Scattering from homogeneous particles in the dilute approximation. Ellipsoids $\{8,8,40\} \AA$ oriented in the $x_{3}$ direction. 
demonstrated that such a model describes large void regions in a-Ge films that give rise to an anisotropic structure factor. The voids are modeled as elongated ellipsoids with the elongated axis in the direction of growth. With the ellipsoids oriented so that $R_{3}$ is along the $x_{3}$-direction, the average is only over the azimuthal angle and thus:

$$
S\left(k, \theta_{k}\right)=\rho_{p} v^{2} V_{e}^{2} \int_{0}^{2 \pi} \Phi^{2}\left(k \sqrt{R_{1}^{2} \cos ^{2} \varphi_{k} \sin ^{2} \theta_{k}+R_{2}^{2} \sin ^{2} \varphi_{k} \sin ^{2} \theta_{k}+R_{3}^{2} \cos ^{2} \theta_{k}}\right) \frac{d \varphi_{k}}{2 \pi},
$$

which reduces to

$$
S\left(k, \theta_{k}\right)=\rho_{p} v^{2} V_{e}^{2} \Phi^{2}\left(k \sqrt{R_{1}^{2} \sin ^{2} \theta_{k}+R_{3}^{2} \cos ^{2} \theta_{k}}\right)
$$

for the case of $R_{1}-R_{2} \oplus R_{3}$. We will work mostly in this approximation, since there is an assumed isotropy in the $x_{1}-x_{2}$ directions, and moreover, it allows one to analytically introduce interparticle effects later.

Regardless of the ellipsoid's relative dimensions, radial scans in $\vec{k}$ show a monotonically decreasing $S\left(k, \theta_{k}\right)$ starting from $k=0$ (see figure 6.3). There are, of course, large $k$ ripples which are from unphysically sharp boundaries between particle and matrix and are introduced by the Fourier transform; these, however, cannot explain the large maximum of the observed scattering.

\section{2.b Inhomogeneous Single Particles}

On the other hand, inhomogeneous particle models 5 have been used in the past to explain such a peak in spherically symmetric systems. A simple modification to the previous isotropic models can be made for ellipsoids and is presented in this section. Suppose that there are two regions of homogeneity in a particle of volume $V_{2}$. The first 

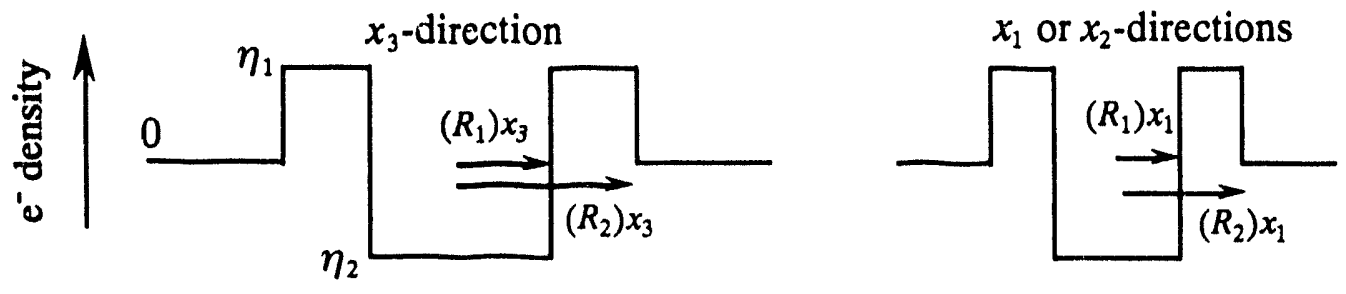

\section{average \\ density}
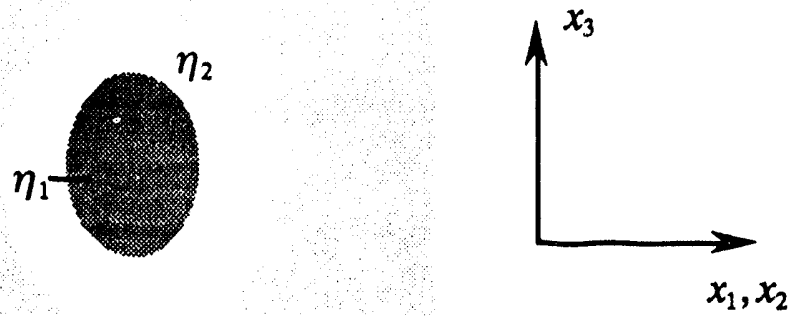

Figure 6.4. Schematic of inhomogeneous particle model. 
occupies a volume $V_{1}$ and has a higher (lower) electron density $\left(\eta_{1}\right)$ than the average, and the other occupies a volume $\left(V_{2}-V_{1}\right)$ with lower (higher) electron density $\left(\eta_{2}\right)$ than average. Then,

$$
F_{\text {inhomo }}(\vec{k})=\eta_{1} \int_{V_{1}} e^{-i \vec{k} \cdot \vec{x}} d^{3} x+\eta_{2} \int_{V_{2}-V_{1}} e^{-i \vec{k} \cdot \vec{x}} d^{3} x .
$$

If the higher density region is deep inside and the lower density along the outer skirts of the particle, as in two confocal ellipsoids (see figure 6.4), then the physical picture of the model is one where (e.g. Fe) atoms migrate to a center of high electron density and leave behind a depletion zone of lower density. The form factor for a confocal model is thus:

$$
F_{\text {inhomo }}(\bar{k})=\left(\eta_{1}-\eta_{2}\right) \Phi\left(k R_{e 1}\right) V_{1}+\eta_{2} \Phi\left(k R_{e 2}\right) V_{2} \text {. }
$$

The observed structure factor is then

$$
S\left(k, \theta_{k}\right)=\rho_{p} \int_{0}^{2 \pi}\left|F_{\text {inhomo }}\left(k, \theta_{k}, \varphi_{k}\right)\right|^{2} \frac{d \varphi_{k}}{2 \pi} .
$$

This equation assumes that the observed scattering from a collection of composite particles is determined by summing the scattering from each particle individually; that is, the particles scatter incoherently with respect to each other (the second term in Eq. 6.2.3 is neglected). This is not a bad assumption, however, since the CCFs (figures 4.10 and 5.3) indicate that there is no appreciable positive correlation surrounding the intraparticle peak. There is, however, a large negative region, which represents the excluded volume, that is automatically taken into consideration with this model. In fact, if there is only little correlation between the composite particles, as the CCFs suggest, this model includes the 
most important features of the correlation function: a well-defined intraparticle contribution with a depletion region. Even if interparticle corielations do exist, the scattering factor of each composite particle is set-up so that it is going to zero at small angles. Then, there is no scattering with which to interfere at small angles and the interparticle interference which causes deviations only at the smaller angles (see next Section) of the composite particles can be neglected; that is, each particle is essentially invisible to its neighbors at low scattering angles.

In order to compare this model with the amorphous films, consider the case where the metal atoms (Fe, Mo) migrate to the center and an amorphous intermetallic occupies the inner ellipsoid. The depletion zone is then a-Ge. The electron densities and volumes of the inner ellipsoids and depletion zones must be self-consistent with the overall composition of the film. For an amorphous alloy of metal concentration $\mathrm{x}$ with an inner region of $\mathrm{FeGe}_{2}$ and depletion zone of a-Ge, we thus have

$$
x=\frac{n_{\text {metal }}^{1} V_{1}}{\left(n_{\text {metal }}^{1}+n_{G e}\right) V_{1}+n_{G e}\left(V_{2}-V_{1}\right)},
$$

where $n_{\text {metal }}^{1}$ is the number density of the metal atoms in volume $V_{1}$ and the Ge number density is assumed to be the same in each region. Since $n_{G e}=2 n_{F e}$ for $\mathrm{FeGe}_{2}$, Eq. 6.2.11 can be simplified to

$$
\frac{V_{1}}{V_{2}}=2 \frac{x}{1-x}
$$

The relative volumes of the ellipsoids are thus determined by the fixed sample composition and the density of the phase separating species. 
For a composition of 6.3 at.\% Fe, ellipsoids of inner axes $R_{1}=(8.5,12.5) \AA$ and outer axes $R_{2}=(15.5,15) \AA$ are in the appropriate size range and give a good reproduction of the observed anisotropy (see figure 6.5a). To achieve the same magnitude of scattering as that observed, the inhomogeneous particle density must be $\sim 5.6 \cdot 10^{-5} \frac{\text { particles }}{\AA^{j}}$, which fills $84 \%$ of the sample volume with composite particles. For the 13.6 at.\% $\mathrm{Fe}$ sample, ellipsoids of $(11,15.5) \AA$ and $(15.5,17.5) \AA$ reproduce the main features of the anisotropy and amplitude change with $\theta_{k}$ (see figure 6.5b). With the composite particles packed together in order to reproduce the magnitude of scattering, the depletion zones (i.e. the region spanned by the difference between the ellipsoids) are touching if not slightly overlapping in some cases. It is important to observe that the depletion zones are more extended in the film plane than in the growth direction. That is, to observe an appreciable SAXS maximum, the relative size of the depletion zone must be greater for an in-plane direction than, if even needed, in the growth direction.

If the inner region is a-MoGe ${ }_{3}, n_{G e}$ is equal to $3 n_{M o}$. Eq. 6.2.11 becomes

$$
\frac{V_{1}}{V_{2}}=3 \frac{x}{1-x}
$$

Ellipsoids of $(8,10) \AA$ and $(12,13.8) \AA$ reproduce the 6.5 at.\% Mo scattering patterns and must be packed to fill $68 \%$ of the available volume to reproduce the observed scattering magnitude (see figure 6.6). Another example is one with the metal atoms located in the depletion zone, with the inner ellipsoid a-Ge. The order-of-magnitude results are similar and will not be discussed further.

The physical picture that thus emerges from this model is one where the particles are formed within the layers by depleting the surrounding region. Then, fluctuations in the deposition process lead to little correlation between where the particles are formed in one 

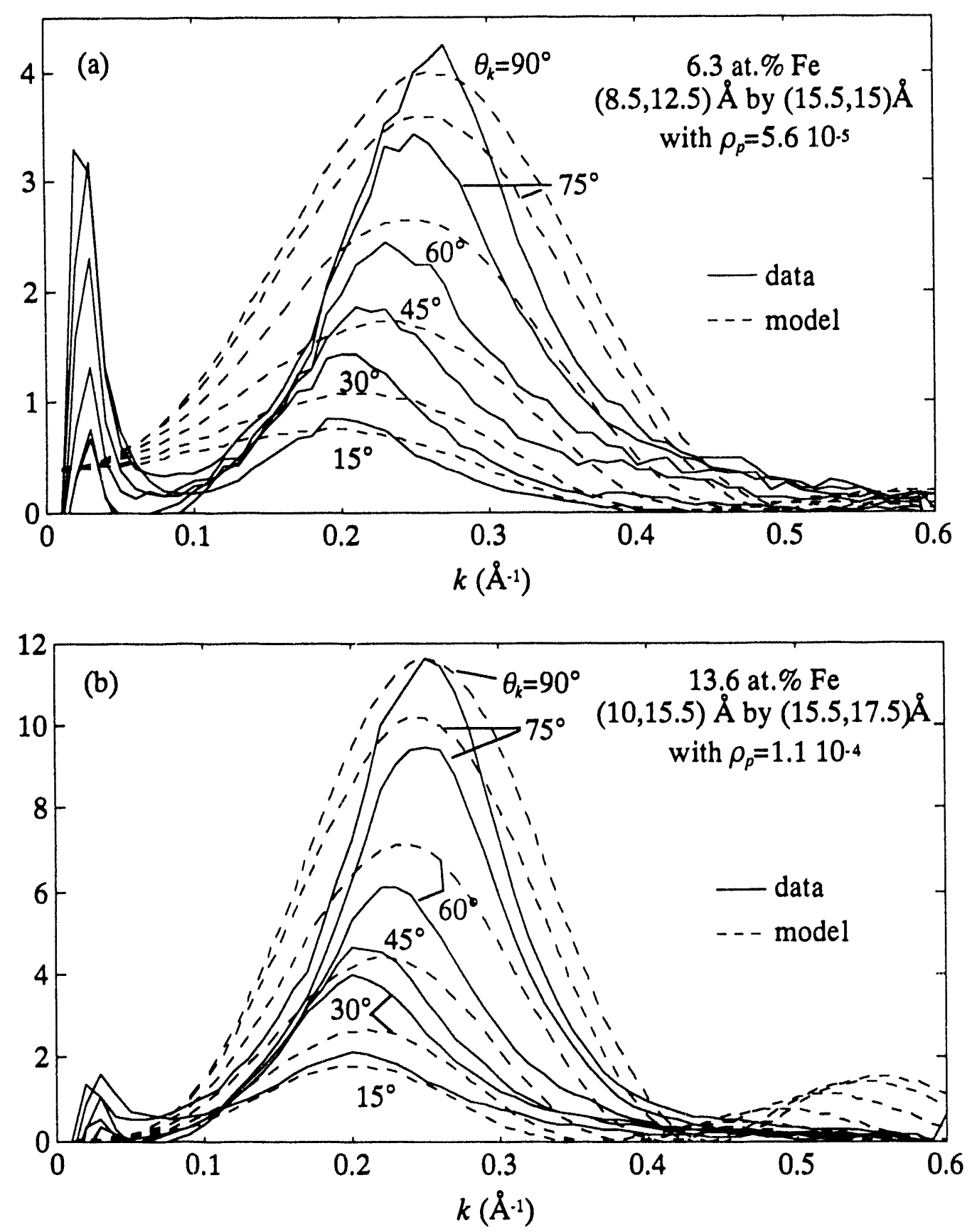

Figure 6.5. Spectra from inhomogeneous particle model as compared to data from (a) the 6.5 at.\% Fe sample and (b) the 13.6 at.\% Fe sample. Change in peak position and amplitude with angle agrees well with data from amorphous films. The electron densities used for these calculations are $(E \sim 6912 \mathrm{eV}): \rho\left(\mathrm{FeGe}_{2}\right)=1.782 \mathrm{e} / \AA^{3}, \rho(\mathrm{Ge})=1.303 \mathrm{e} / \AA^{3}$, $\rho\left(\mathrm{x}_{\mathrm{Fe}}=0.063\right)=1.404 \mathrm{e} / \AA^{3}$ and $\rho\left(\mathrm{x}_{\mathrm{Fe}}=0.136\right)=1.515 \mathrm{e} / \AA^{3}$. 


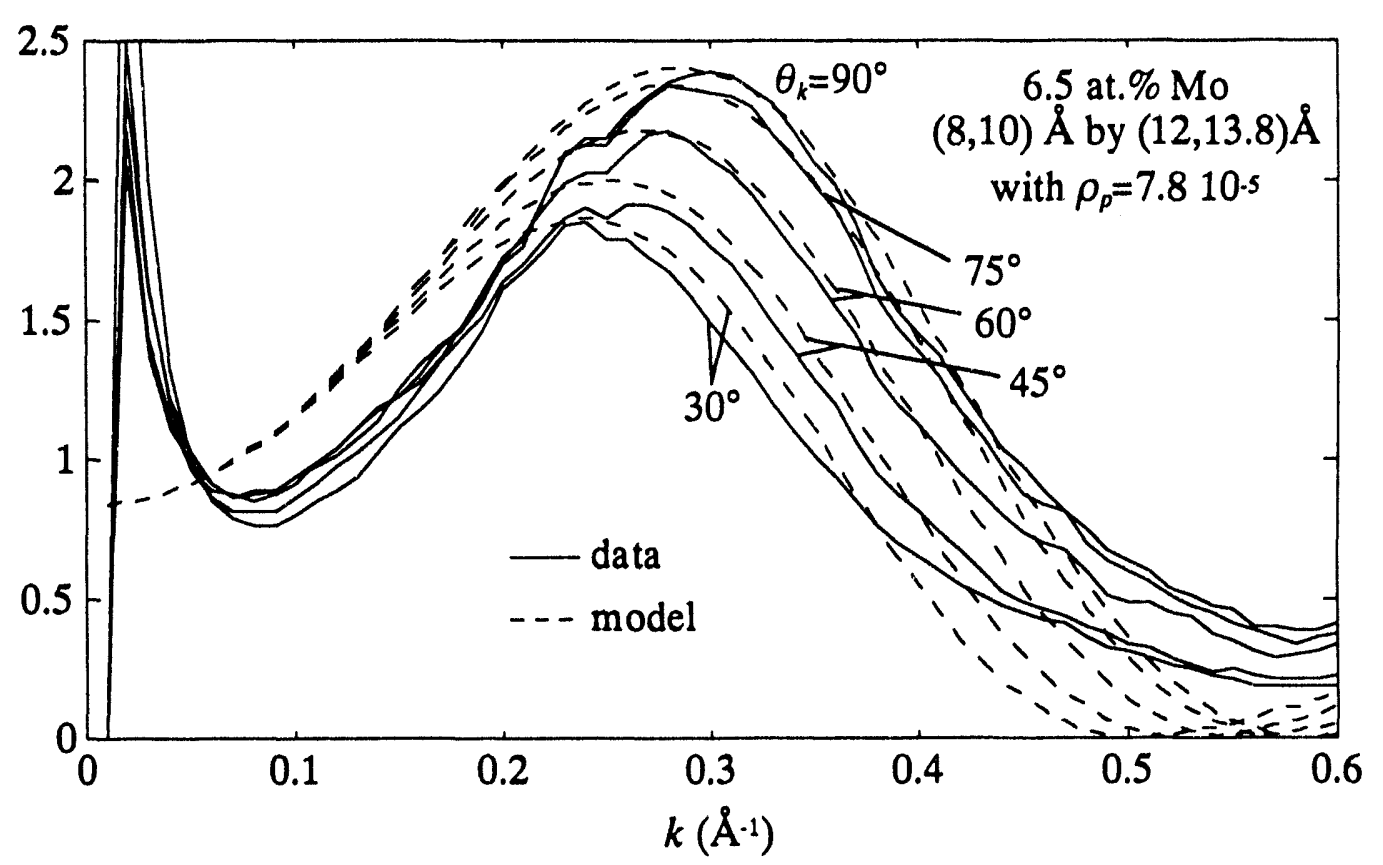

Figure 6.6. Spectra from inhomogeneous particle model as compared to data from the 6.5 at.\% Mo sample. The electron densities used for these calculations are $(E \sim 7100 \mathrm{eV})$ : $\rho\left(\mathrm{MoGe}_{3}\right)=1.896 \mathrm{e} \cdot / \AA^{3}, \rho(\mathrm{Ge})=1.303 \mathrm{e} / \AA^{3}$, and $\rho\left(\mathrm{x}_{\mathrm{M}_{0}}=0.065\right)=1.453 \mathrm{e} / \AA^{3}$. 
layer and where they are formed after about $20 \AA$ further in the growth direction. This is consistent with the CCFs which show little, if any, negative region in the growth direction but a large negative region for the in-plane direction. Of course this model assumes that the scattering from each particle adds incoherently (i.e. no interparticle interference), but, as mentioned previously, this is not a bad assumption at low scattering angles since such interference will not be great.

\subsection{Close-Packed Homogeneous Particles}

The 20 to $30 \AA$ spacings from the inhomogeneous particle model, with the outer depletion zones touching, leads one to imagine another model that consists of ellipsoids in an interconnected depletion zone: a close-packing of homogeneous ellipsoids in a matrix of constant electron density. Since the mean-square fluctuation in density calculation identified two electron densities, that of a-Ge and either a-FeGe $\mathrm{Fe}_{2}$ a- $-\mathrm{MoGe}_{3}$, we imagine particles of the Ge-rich intermetallic in a matrix of a-Ge. When the volume fraction of intermetallic phase exceeds 0.5 , then the particles are more likely a-Ge, with the matrix the intermetallic phase.

Calculating the interparticle interference function for a system of interacting particles is difficult, and several simplifying assumptions are required. One approach to the problem of interparticle interference and anisotropy is to assume that the material consists of orienied, homogeneous, identical ellipsoids. This is the simplest approach one can take to the anisotropy, yet it still provides a deep understanding of the material's structure and permits the use of exact, analytic functions to describe its properties (e.g. pair correlation functions). Polydispersity complicates matters but, if it is not too great, does not significantly alter the main peak positions and average particle sizes. ${ }^{6}$ For the study of close-packed particles, the fundamental assumption we will make is that all particles are identical, are ellipsoidal in shape $\left(R_{1}=R_{2}\right)$, and are oriented such that $R_{3}$ aligns with $x_{3}$. 
The goal of this section is to apply equilibrium liquid theory to derive an interparticle interference function. First, general relations between the interparticle correlation functions are discussed and then an interparticle interference function is derived for oriented ellipsoids in the hard-core Percus-Yevick approximation.

\section{3.a. Relations Between Interparticle Correlation Functions}

Since the particles are identical (in orientation and size), the averaging of the particle positions can be separated from that of particle size and orientation:

$$
S(\bar{k})=\rho_{p}\left\langle|F(\bar{k})|^{2}\right\rangle+\frac{1}{V}|\langle F(\bar{k})\rangle|^{2}\left\langle\sum_{m} \sum_{n=m} e^{-i \bar{k} \cdot\left(\bar{x}_{m}-\bar{x}_{n}\right)}\right\rangle
$$

Moreover, with

$$
F^{2}(k, \theta)=\left\langle|F(\bar{k})|^{2}\right\rangle=|\langle F(\bar{k})\rangle|^{2}=v^{2} \Phi^{2}\left(k \sqrt{R_{1}^{2} \sin ^{2} \theta_{k}+R_{3}^{2} \cos ^{2} \theta_{k}}\right) V_{e}^{2},
$$

the total structure factor is thus

$$
S(k, \theta)=\rho_{p} F^{2}\left(k, \theta_{k}\right)\left(1+\frac{1}{N}\left\langle\sum_{m} \sum_{n=m}^{-i k \cdot \ell_{m m}}\right\rangle\right)
$$

with $\bar{X}_{m n}=\bar{X}_{m}-\bar{X}_{n}$. Eq. 6.3 .3 shows that it is possible, albeit with assumptions, to separate the scattering of the particles (first term) from the interparticle interference effects (second term) which we will define as the function $S_{I N T}$. This function is tricky to evaluate. Since the particles are elongated, it is not possible to perform a simple spherical average over all equivalent particle positions as is often done in liquids or amorphous solids. That is, the probability of finding a particle a certain distance along axis $R_{3}$ will differ from the 
probability of finding a particle that same distance but along $R_{1}$. Rather, the locus of points which map the equivalent positions of particles is an ellipsoid determined by the size of particles and interparticle potentials.

Eq. 6.3.3 can be further simplified by introducing a probability function, $g_{m}\left(\bar{X}_{m n}\right)$, such that $\rho_{p} g_{m}\left(\bar{X}_{m n}\right) d V_{n}$ is the number of ellipsoid centers that are occupied in the volume element $d V_{n}$ at $\bar{X}_{m n}$ relative to particle $m$. With $g_{m}\left(\bar{X}_{m n}\right)$, the sum over $n$ is replaced by an integral:

$$
S_{I N T}(\bar{k})=1+\frac{1}{V}\left\langle\sum_{m} \int_{V} g_{m}\left(\bar{X}_{m n}\right) e^{-i \bar{k} \cdot X_{m}} d V_{n}\right\rangle
$$

For a given displacement $\vec{X}-\bar{X}_{m n}$, the radial distribution function is defined as $g(\bar{X})=\left\langle g_{m}\left(\bar{X}_{m n}\right)\right\rangle$. The average is performed over all positions of particles $m$ for each vector $\bar{X}$. For a model of disordered particles, $g(\bar{X})$ tends to 1 for distances a few times that of the size of the ellipsoid, and hence the sum over $m$ can be replaced with $N$. The result:

$$
S_{I N T}(\vec{k})=1+\rho_{p} \int_{V}(g(\bar{X})-1) e^{-i \vec{k} \cdot \bar{x}} d V+\rho_{p} \int_{V} e^{-i k \cdot \cdot \bar{x}} d V
$$

where a constant term has been added and subtracted to the integrand. In the large volume limit, the third term is omitted since its contribution is obscured by the direct beam and not directly measurable. The final relation is thus:

$$
S_{I N T}(\bar{k})=1+\rho_{p} \int_{V}(g(\bar{X})-1) e^{-i \bar{k} \cdot \bar{x}} d V
$$


Eq. 6.3.6 is the usual Fourier transform relation between the radial distribution function and the interparticle interference function.

It is important to notice that the integrand, $g(\bar{X})-1$, is also often referred to as the total correlation function, $h(\bar{X})$, by the Ornstein-Zernike integral relations. ${ }^{7}$ In the Ornstein-Zernike formalism, the total pair correlation function is the sum of two terms: (1) the direct term or direct correlation function, $c(\bar{X})$, between two particles, and (2) an indirect term that consists of the influence of all other particles on the correlation between the two particles:

$$
h(\bar{X})=c(\bar{X})+\rho_{p} \int_{V^{\prime}} c\left(\bar{X}-\bar{X}^{\prime}\right) h\left(\bar{X}^{\prime}\right) d V^{\prime}
$$

Since the radial distribution and total correlation functions are simply related, applying the Fourier convolution theorem to Eq. 6.3.7 and combining with Eq. 6.3.6 leads to the important relation between the Fourier transform of the direct correlation function, $\vec{c}(\vec{k})$, and $S_{I N T}(\bar{k})$ :

$$
S_{I N T}(\bar{k})=\frac{1}{1-\rho_{p} \bar{c}(\bar{k})}
$$

The problem is a matter of finding a suitable direct correlation function for a model of close-packed particles; the interparticle interference function can then be obtained from Eq. 6.3.8 and, hence, the model's scattering pattern.

\section{3.b Hard-Core Percus-Yevick Approximation}

Theories of the structure of liquids rely on accurate knowledge of an assumed interparticle potential energy function. The particles, then, rearrange until a minimum of 
free energy is obtained. Although it is not obvious that the equilibrium theory of liquids can be applied to the special case of a disordered vapor-deposited film, it is the only means available to calculate an interparticle interference function for an interacting system of particles. The extent to which the particles interact define the thermodynamic properties of the fluid and, hence, its structure. One of the most successful theories of liquid structure, especially for liquid metals, ${ }^{8}$ is the hard-core Percus-Yevick model which has an analytic solution for spheres. Agreement with Lennard-Jones fluids ${ }^{9}$ and consistency between the compressibility and pressure equations of state ${ }^{10}$ has led to its widespread use. Despite the purely repulsive interparticle potential energy function, it is permissible to ignore the attractive components as a first approximation, since at high density the repulsive features dominate.

The direct correlation function has been determined in the Percus-Yevick approximation for a hard-core repulsive potential that is ellipsoidal in shape. It should be stressed that the hard core ellipsoid is not necessarily identical to that of the particle ellipsoid. As noted by Pederson ${ }^{6}$ in the study of spherical precipitates in alloys, precipitates incorporate the surrounding material, and these outer regions thus define an effective hard core repulsion of somewhat larger distances than those that allow the particles to touch. Of course in the study of inert gases or liquid metals with a true hard core repulsion, the hard core permits the closest approach between spheres to equal its diameter.

The center of each hard-core is also the center of its corresponding ellipsoidal particle. The hard core is a short-ranged purely repulsive interparticle interaction, with the following pair potential, $\phi(\vec{X})$ :

$$
\phi(\bar{X})=\begin{aligned}
& \infty, \quad|\bar{X}|<2 \sigma_{\dot{x}} \\
& 0, \quad|\bar{X}|>2 \sigma_{\dot{x}}
\end{aligned}
$$


with $\sigma_{\hat{x}}$ the orientation-dependent distance from the center of the hard core to a point on its surface. For a hard core ellipsoid of axes $\vec{\sigma}-\left(\sigma_{1}, \sigma_{3}\right)$ oriented in the $x_{3}$-direction, $\sigma_{\hat{x}}$ is given by

$$
\sigma_{\dot{x}}=\frac{\sigma_{1} \sigma_{3}}{\sqrt{\sigma_{1}^{2} \cos ^{2} \theta+\sigma_{3}^{2} \sin ^{2} \theta}}
$$

with an assumed $x_{1}-x_{2}$ isotropy. The closest approach between two hard cores is $2 \sigma_{\dot{x}}$, which, as mentioned previously, can be greater than twice the distance between the particles for precipitates in solids.

The Percus-Yevick equation for the general hard-core potential ${ }^{11}$ is given by solving the following integral equation:

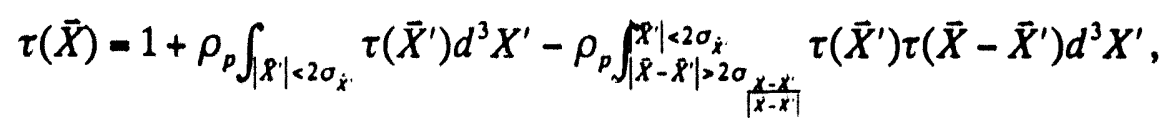

with the new function $\tau(\bar{X})$ defined in terms of the pair correlation functions:

$$
\begin{array}{ll}
|\vec{X}|<2 \sigma_{\dot{\hat{x}}}: & g(\bar{X})=0 \text { and } c(\bar{X})=-\tau(\bar{X}) \\
|\vec{X}|>2 \sigma_{\dot{X}}: & c(\bar{X})=0 \text { and } g(\bar{X})=\tau(\bar{X})
\end{array}
$$

The integral equation has been solved exactly for spherically symmetric particles by Wertheim ${ }^{12}$ and Thiele. ${ }^{13}$ By a transformation of variables, it can be shown that Eq. 6.3.12 can also be solved exactly. With $\vec{y}=\left(\frac{x_{1}}{\sigma_{1}}, \frac{x_{2}}{\sigma_{1}}, \frac{x_{2}}{\sigma_{3}}\right)$ and $\vec{y}^{\prime}=\left(\frac{x_{1}}{\sigma_{x}}, \frac{x_{i}}{\sigma_{x}}, \frac{x_{i}}{\sigma_{2}}\right)$, the PercusYevick equation for the ellipsoids can be transformed to one for spheres of radius $=1$ : 


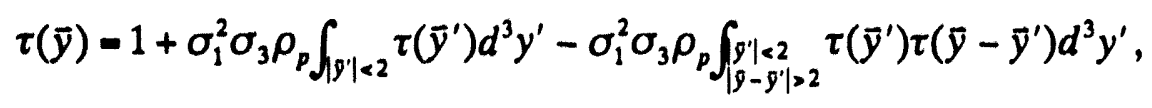

with

$$
\begin{array}{ll}
|\bar{y}| \leq 2: & g(\bar{y})=0 \text { and } c(\bar{y})=-\tau(\bar{y}) \\
|\vec{y}|>2: & c(\bar{y})=0 \text { and } g(\bar{y})=\tau(\bar{y})
\end{array}
$$

This is readily solved by Wertheim and Thiele's approach:

$$
c(\vec{y} \leq 2)-\frac{-1}{(1-\eta)^{4}}\left[(1+2 \eta)^{2}-6 \eta(1+\eta / 2)^{2}|\vec{y}|+\frac{\eta}{2}(1+2 \eta)^{2}|\vec{y}|^{3}\right],
$$

with the packing fraction $\eta=V_{\sigma} \rho_{p}=\frac{4}{3} \pi \sigma_{1}^{2} \sigma_{3} \rho_{p}$. Eq. 6.3.14 is identical to

$$
c\left(\bar{X} \leq 2 \sigma_{\dot{\chi}}\right)=\frac{-1}{(1-\eta)^{4}}\left[(1+2 \eta)^{2}-6 \eta(1+\eta / 2)^{2} \frac{|\not|}{2 \sigma_{\ell}}+\frac{\eta}{2}(1+2 \eta)^{2} \frac{|\not|^{3}}{\left(2 \sigma_{\ell}\right)^{3}}\right]
$$

a form first proposed by Pynn ${ }^{14}$ for elongated particles, but with a different $\eta$, that has been used in the study of the isotropic-nematic transitions in the hard ellipsoid fluid. ${ }^{15}$ In these studies, the ellipsoids are oriented at various angles with respect to each other, so that Eq. 6.3.15 is taken only as an approximation. For ellipsoids of various orientation, the distance of closest approach is a complicated function that is simplified by the Berne and Pechukas ${ }^{16}$ modification. This theory breaks down, however, when the eccentricity of the ellipsoids exceeds $1.5-2$ or when the ellipsoids align in the $\sigma_{3}$-direction. It should be pointed out, of course, that numerical studies have been performed that use a spherical harmonics approach with the correct closest approach distance. ${ }^{17}$ For ellipsoids oriented in one direction as in the model presented here, the distance of closest approach between two hard cores is precisely $2 \sigma_{\hat{\boldsymbol{x}}}$, and the derived solution (Eq. 6.3.15) is exact. 
The Fourier transform of $c(\vec{X})$ can be expressed in terms of elementary functions so that an analytic expression for $S_{I N T}(\bar{k})$ is possible. The Fourier transform of the direct correlation function is

$$
-\tilde{c}(\bar{k})=\int_{|\bar{x}|<2 \sigma_{\dot{x}}}\left(\alpha+\beta \frac{|\bar{x}|}{2 \sigma_{\dot{x}}}+\delta \frac{|\bar{x}|^{3}}{\left(2 \sigma_{\dot{x}}\right)}\right) e^{-i \vec{k} \cdot \dot{x}} d^{3} X,
$$

where $\alpha=\frac{(1+2 \eta)^{2}}{(1-\eta)^{4}}, \beta=-\frac{6 \eta(1+\eta / 2)^{2}}{(1-\eta)^{4}}$, and $\delta=\frac{\eta}{2} \frac{(1+2 \eta)^{2}}{(1-\eta)^{4}}$.

With a transformation of variables (e.g. as in Eq. 6.2.5), the integral over the hard core is replaced by one over the unit sphere:

$$
-\bar{c}(\vec{k})=8 \sigma_{1}^{2} \sigma_{3} \int_{\text {sphit }}^{\text {unit }}\left(\alpha+\beta x+\delta x^{3}\right) \exp (-i \overrightarrow{\vec{k}} \cdot \vec{x}) d^{3} x
$$

with $\overrightarrow{\tilde{k}}=2\left(k_{1} \sigma_{1}, k_{2} \sigma_{1}, k_{3} \sigma_{3}\right)$. This can be readily integrated in terms of elementary trigonometric functions (left to the reader), so that the interparticle interference becomes:

$$
S_{I N T}(\vec{k})=\frac{1}{1+\frac{24}{\tilde{k}} \eta \int_{0}^{1}\left(\alpha+\beta x+\delta x^{3}\right) \sin (\tilde{k} x) x d x}
$$

Figure 6.7 illustrates $S_{I N T}(\bar{k})$ for hard core ellipsoids $(10,15) \AA$ with a packing fraction $\eta=0.35$.

It should be emphasized that the interparticle potential is defined by the hard core ellipsoid. The position of one hard core with respect to another is considered equally probable over that locus of points defined by the second hard core. In an amorphous film, however, there is no real interaction between particles, and the Percus-Yevick approach 


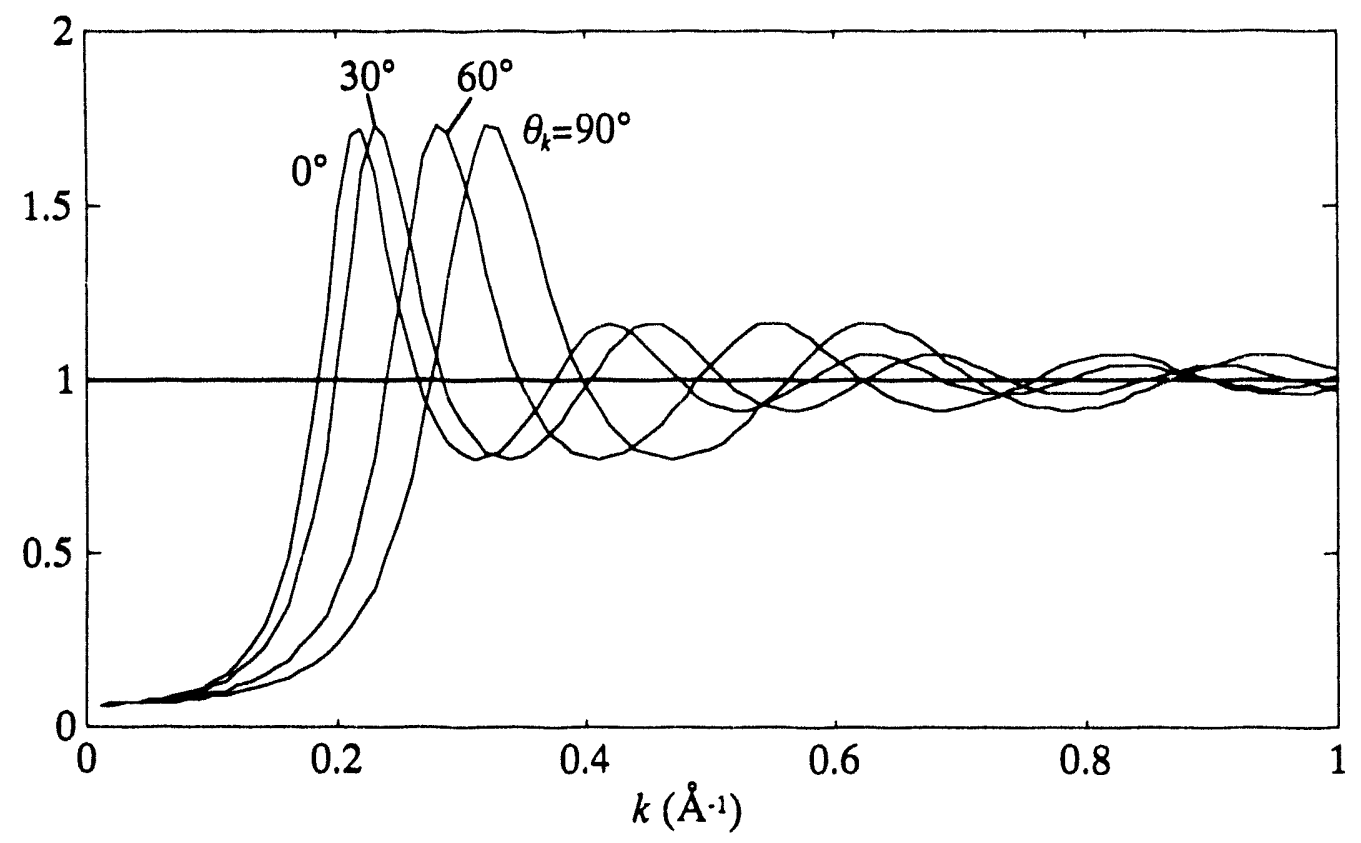

Figure 6.7. Interparticle interference function, $S_{I N T}\left(k, \theta_{k}\right)$, in the Percus-Yevick approximation for oriented ellipsoids of $\{10,15\} \AA$ with a packing fraction of 0.35 . If there is no interparticle interference, then $S_{I N T}=1$. The line drawn for $S_{I N T}=1$ show that the interparticle interference effects are greatest at small $k$. 
with a fictitious hard core interaction is used only to arrive at an analytic expression of the interparticle interference function. The maximum amplitude of the Percus-Yevick $S_{I N T}\left(k, \theta_{k}\right)$ depends only on $\eta$ and does not change with $\theta_{k}$ (see figure 6.7). It will be shown later that this is one difference that is noticeable when compared to the interparticle function that we extract from the data. In the films, the maximum amplitude of the interparticle function appears to be a function of the film direction.

\section{3.c Results of Model}

In this section, we report the basic results of the close-packed ellipsoid model. The packing fraction, particle density, and particle and hard core ellipsoid axes are related by the overall film composition. For a model of phase separation with the Ge-rich intermetallic phase (e.g. $\mathrm{FeGe}_{2}$ or $\mathrm{MoGe}_{3}$ ) as the particle in a matrix of a-Ge, the concentration of metal atoms is given by:

$$
x=\frac{V_{e} \rho_{p} n_{\text {metal }}}{V_{e} \rho_{p} n_{\text {metal }}+n_{G e}},
$$

with $V_{e}$ and $V_{\sigma}$ the volumes of the ellipsoidal particle and hard core, respectively. The packing fraction is consequently $\eta=\left(\frac{x}{1-x}\right) \frac{V_{o}}{V_{e}} \frac{n_{G e}}{n_{\text {meal }}}$.

When the volume fraction of the Ge-rich intermetallic exceeds $1 / 2$, the ellipsoids become a-Ge with the surrounding material the intermetallic. Since the results are similar (Babinet principle), we will focus on the low metal concentration alloys with the metallic phase as the particle. For reference, the volume fraction is $1 / 2$ at 20 at.\% Fe (a-Ge and a$\mathrm{FeGe}_{2}$ ) and 14 at.\% Mo (a-Ge and a-MoGe ${ }_{3}$ ). By choosing reasonable axes for the particle and hard core ellipsoids, the general features of the anisotropic scattering can be reproduced (figures 6.8-6.11). The location of the SAXS maximum, change with tilt, and the order of 

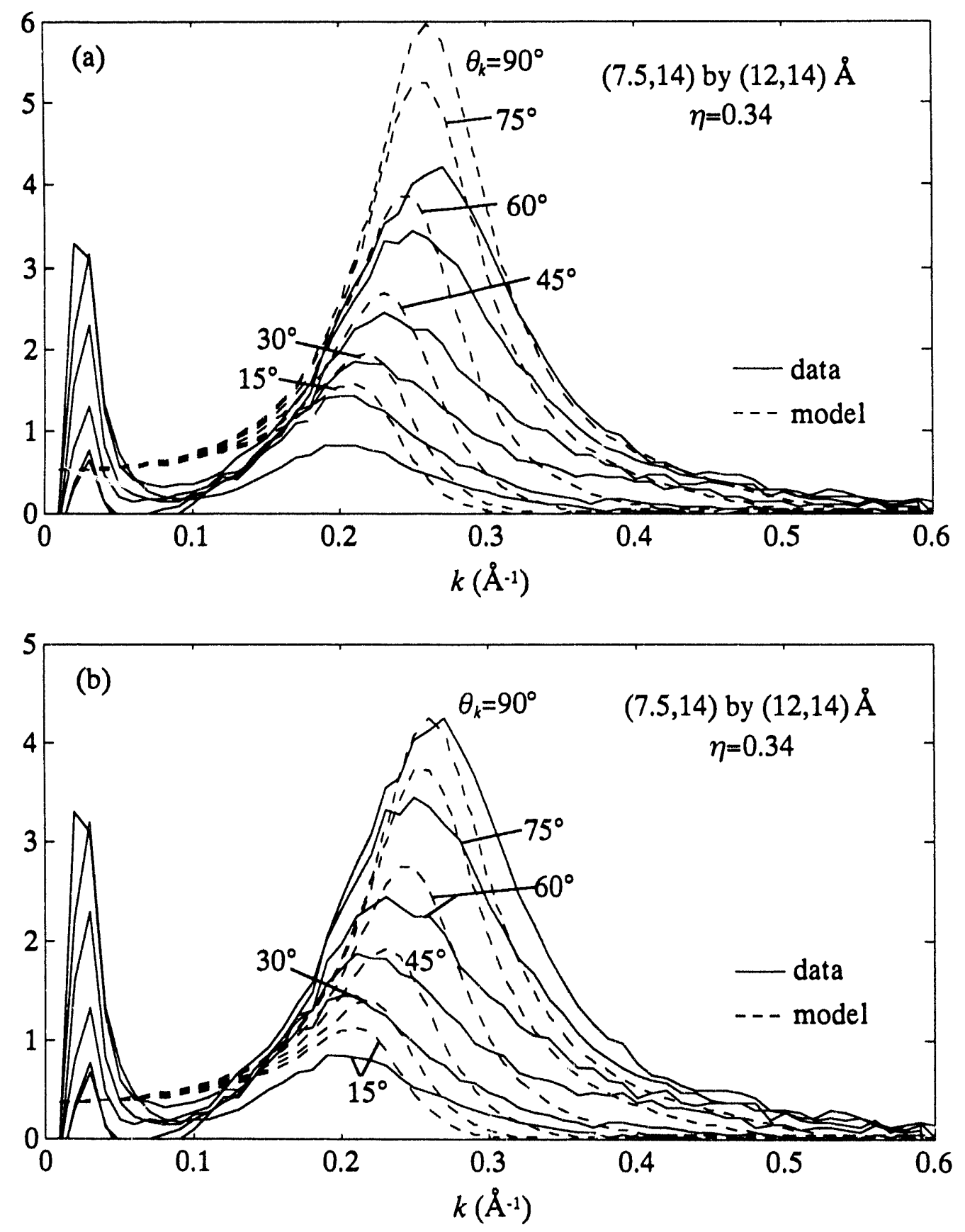

Figure 6.8. $r_{e}{ }^{2} S\left(k, \theta_{k}\right)\left(\mathrm{cm}^{-1}\right)$ for ellipsoids of $(7.5,14)$ by $(12,14) \AA$ (given as particle axes by hard core axes) for a film composition 6.3 at. $\% \mathrm{Fe}$ (with a corresponding packing fraction of 0.34). (a) Comparison of close-packed model with data collected at $6912 \mathrm{eV}$ (electron densities for $\mathrm{Ge}$ and $\mathrm{FeGe}_{2}$ the same as that used in figure 6.5). (b) Model scaled to the maximum at $\theta_{k}=90^{\circ}$ to better observe anisotropy and peak shift. 

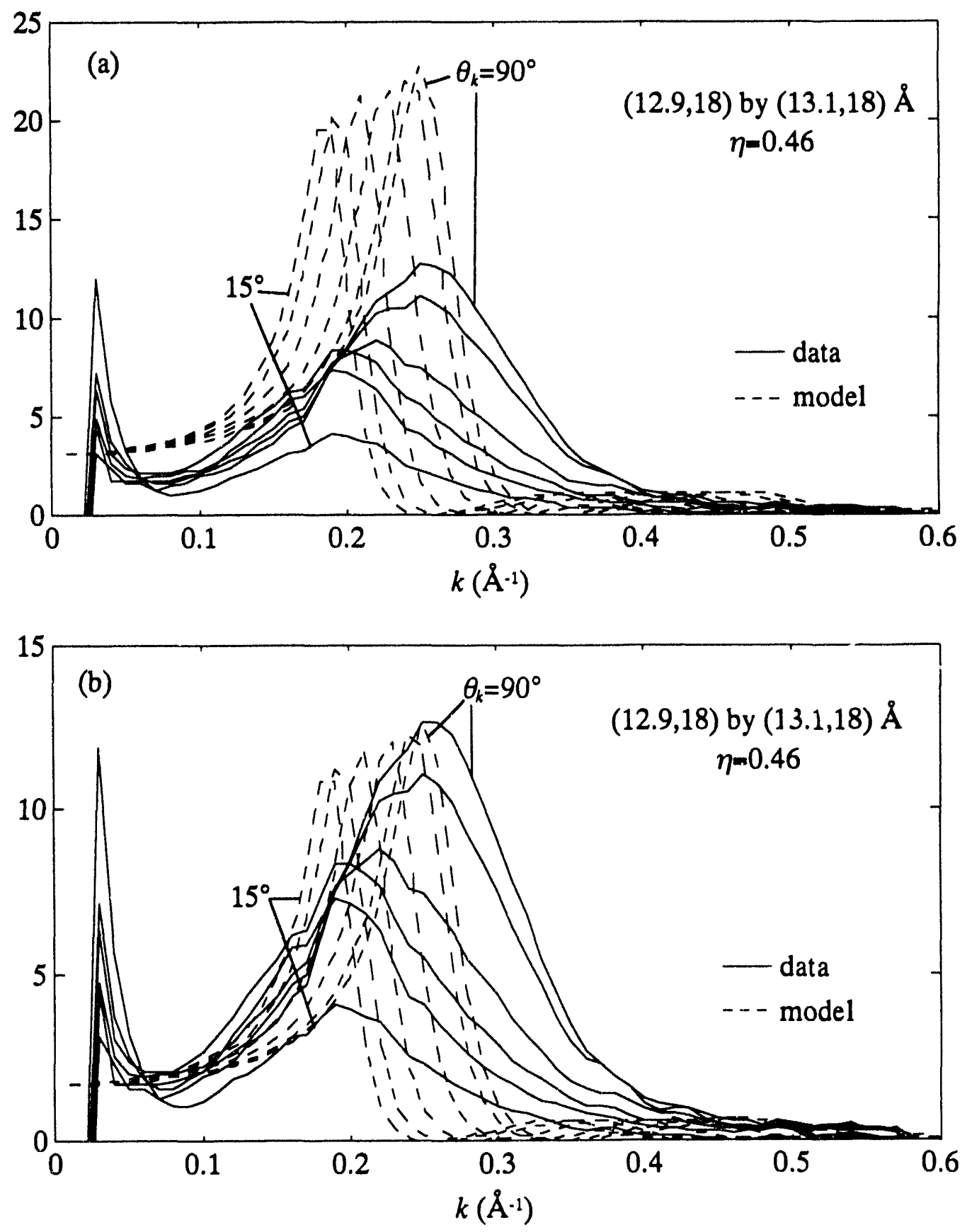

Figure 6.9. $r_{e}^{2} S\left(k, \theta_{k}\right)\left(\mathrm{cm}^{-1}\right)$ for ellipsoids of $(12.9,18)$ by $(13.1,18) \AA$ for a film composition 18.2 at.\% $\mathrm{Fe}$ (with $\eta=0.46$ ). (a) Comparison of close-packed model with data collected at $10903 \mathrm{eV}\left(\rho(\mathrm{Ge})=1.193 \mathrm{e} / \AA^{3}\right.$ and $\left.\rho\left(\mathrm{FeGe}_{2}\right)=1.737 \mathrm{e} / \AA^{3}\right)$. (b) Model scaled to the maximum at $\theta_{k}=90^{\circ}$ to better observe anisotropy and peak shift. 

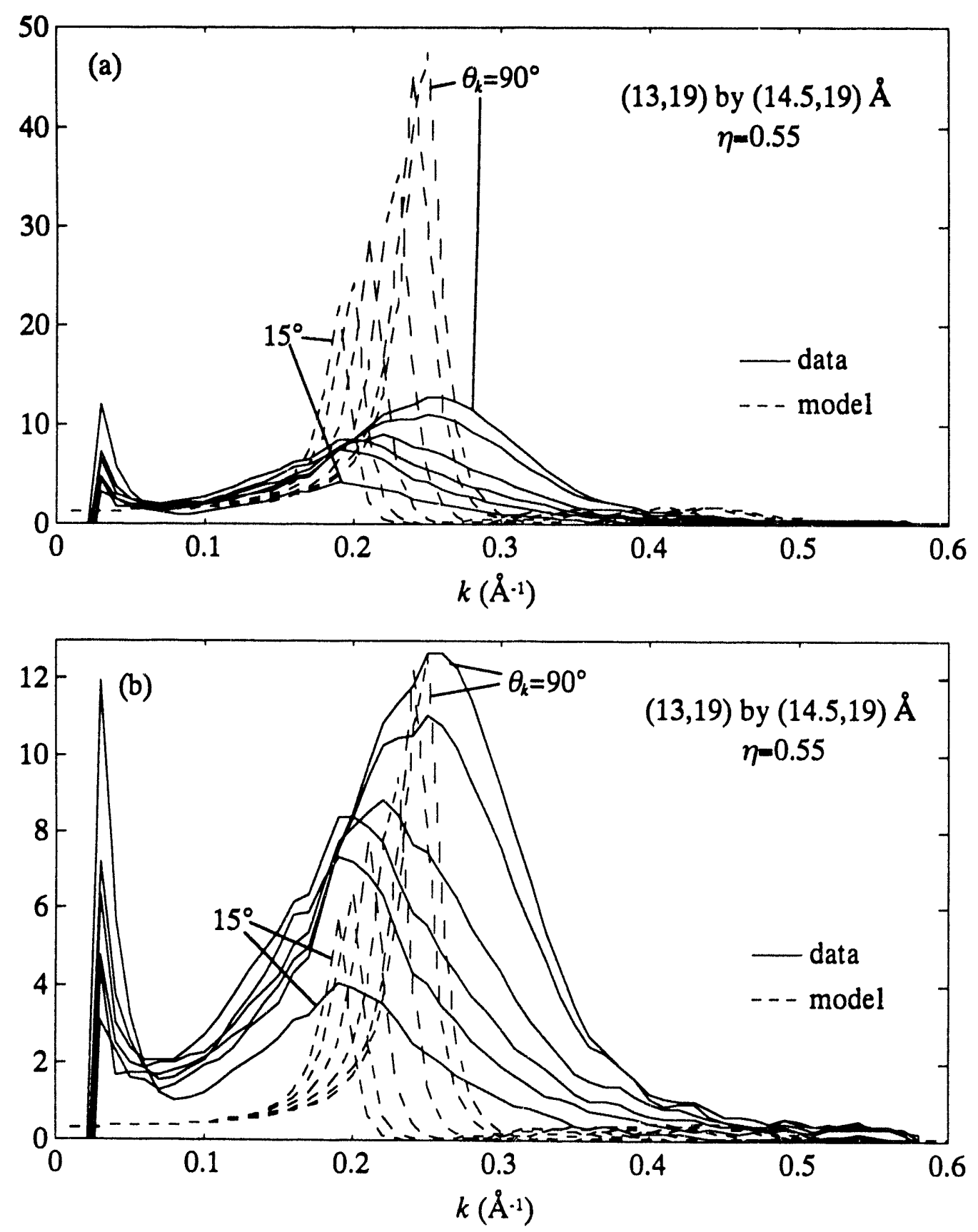

Figure 6.10. $\quad r_{e}{ }^{2} S\left(k, \theta_{k}\right)\left(\mathrm{cm}^{-1}\right)$ for ellipsoids of $(13,19)$ by $(14.5,19) \AA$ for a film composition 18.2 at.\% $\mathrm{Fe}$ (with $\eta=0.55$ ). (a) Comparison of close-packed model with data collected at $10903 \mathrm{eV}$ (electron densities same as in figure 6.9). (b) Model scaled to the maximum at $\theta_{k}=90^{\circ}$ to better observe anisotropy and peak shift. 

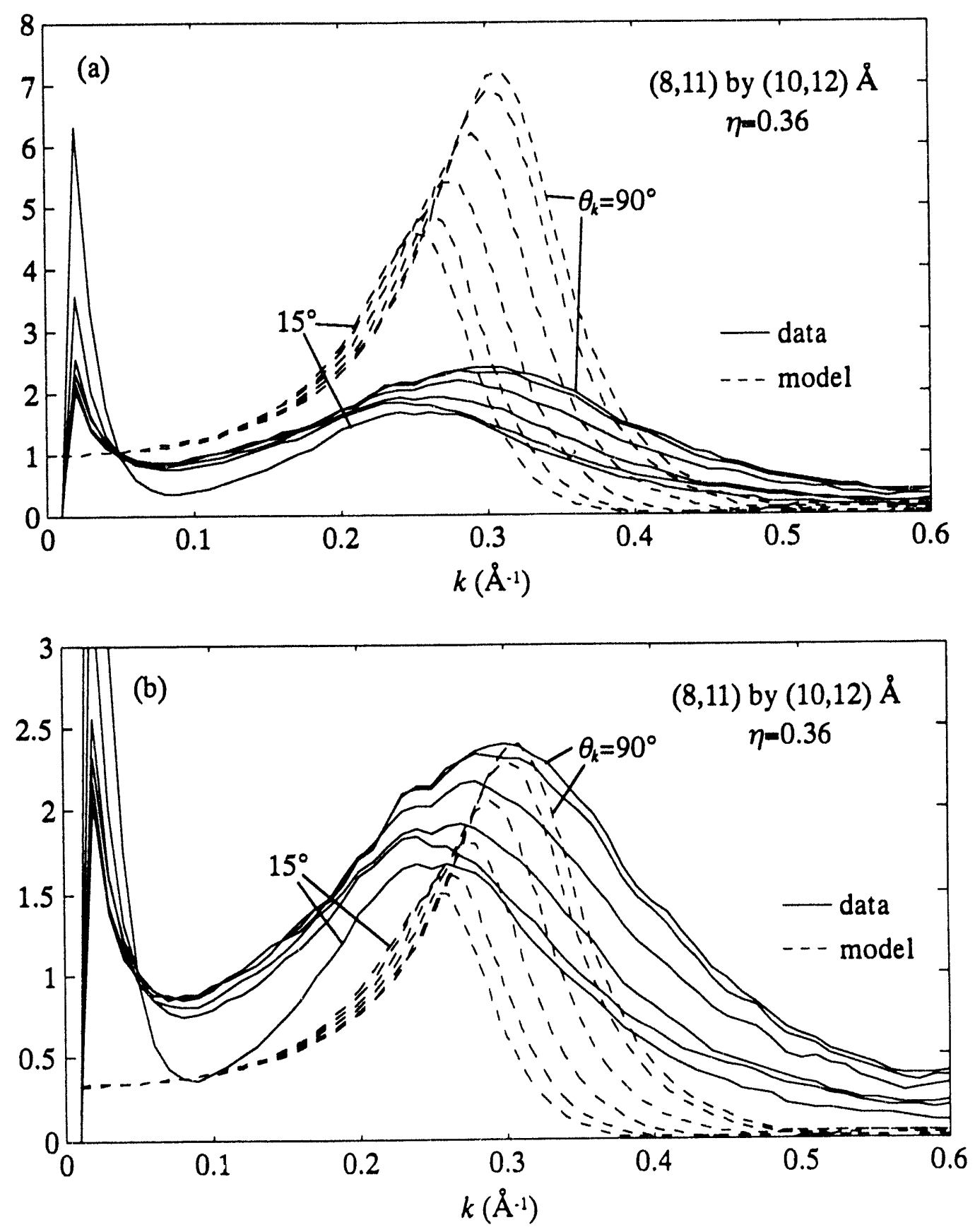

Figure 6.11. $r_{e}{ }^{2} S\left(k, \theta_{k}\right)\left(\mathrm{cm}^{-1}\right)$ for ellipsoids of $(8,11)$ by $(10,12) \AA$ (given as particle axes by hard core axes) for a film composition 6.5 at.\% Mo (with a corresponding packing fraction of 0.36). (a) Comparison of close-packed model with data collected at $7100 \mathrm{eV}$ (electron densities for $\mathrm{Ge}$ and $\mathrm{MoGe}_{3}$ the same as that used in figure 6.6). (b) Model scaled to the maximum at $\theta_{k}=90^{\circ}$ to better observe anisotropy and peak shift. 
magnitude of scattering are in agreement with the observed scattering for both the Mo and Fe based alloys.

For a 6.3 at.\% Fe sample, an ellipsoid with axes $(7.5,14)$ and $(12,14) \AA$ with a corresponding packing fraction $\eta=0.34$ works well (see figure 6.8 --these values chosen to reproduce the peak position at each measured $\theta_{k}$ ). There is a limit to the general features that the model can reproduce, however, especially at large packing fractions, and is the reason we have chosen not to "best-fit" the observed spectra to the model. The magnitude of scattering and breadth of the maximum depend critically on the shape of the PercusYevick interparticle function and the chosen packing fraction. The FWHM is too sharp and the magnitude of scattering is always too large to fit the data. It is expected that with a polydisperse model, the maximum would "wash out"; that is, the FWHM would increase with a corresponding decrease in the peak amplitude. No polydisperse model of oriented ellipsoids exists, however, to test our ideas. The agreement between model and theory is still remarkable, however, from the order of magnitude of scattering to the reproduction of the peak position. This is a simple monodisperse model with an ideal interparticle interference function.

For samples with even greater $\mathrm{Fe}$ concentrations, the larger packing fractions lead to sharper maxima in the interparticle function. For example, consider the 18.2 at.\% $\mathrm{Fe}$ sample (see figures 6.9-6.10). In order to reproduce the data, the packing fraction in the Percus-Yevick model must not be too great since a large packing fraction leads to interparticle interference maxima that are quite large with narrow FWHMs. With ellipsoids of axes $(12.9,18)$ and $(13.1,18) \AA$ and $\eta=0.46$, the anisotropy of the 18.2 at.\% Fe sample can be reproduced. The relative change in magnitude of scattering with the change in $\theta_{k}$ for these ellipsoids is not good; figure 6.10 shows the comparison between another set of axes $[(13,19)$ by $(14.5,19) \AA]$ and the data. In this case, the relative change in magnitude of scattering is in better agreement with the data, but the larger packing fraction $(\eta=0.55)$ 
leads to very sharp FWHMs that are not observed in the data. This example illustrates the main problem with the model. The monodispersity leads to very sharply defined maxima (even for moderate packing fractions) that are not experimentally observed.

Another interesting feature is the relative difference between the axes of the particle and hard core ellipsoids. If the particle and hard core axes are similar in the growth direction but dissimilar in the plane of the film, then the change in amplitude with tilt angle is that which is experimentally observed (decrease in magnitude as $\theta_{k}$ changes from 90 to $15^{\circ}$ ). Conversely, if the particle and hard core axes are similar in the plane of the film but dissimilar in the growth direction, then the change in amplitude with tilt is exactly opposite (as $\theta_{k}$ changes from 90 to $15^{\circ}$ the SAXS increases). Our results thus indicate that, on average, the particles are not allowed to touch when they are aligned in the plane of the film $\left(R_{1}-R_{2}<\sigma_{1}-\sigma_{2}\right)$ but that they can nearly touch in the growth direction $\left(R_{3} \sim \sigma_{3}\right)$. We might consider this a quasi-columnar structure, with the possibility that the ellipsoids can touch in the growth direction but with well-defined regions of a-Ge between the ellipsoids for the in-plane directions. However, with packing fractions less than $1 / 2$, a true columnar structure cannot be established, as further evidenced from our results in Section 6.1a. This result is also confirmed from the CCF and the inhomogeneous particle models, where a depletion zone is established in the film surface plane but does not appear as important in the growth direction.

Since the computed scattering is the product of $S_{I N T}\left(k, \theta_{k}\right)$ and $F^{2}\left(k, \theta_{k}\right)$, there exist competing factors that influence the amplitude and width of $S\left(k, \theta_{k}\right)$. The magnitude of scattering in figure 6.8 is approximately 1.5 times that observed for the 6.3 at.\% Fe. This is not too surprising, since the model does not include any variations in particle or hard core size and shape. This is in fact the reason why we have not fit the data to the monodisperse model. The sharp magnitude from the Percus-Yevick interparticle interference function at moderate packing fraction is simply too great to reproduce the data. 
To decrease the maximum amplitude in figure 6.8 , it would normally be sufficient to decrease the packing fraction, but the overall film composition must be maintained. Thus, a decrease in $\eta$ implies an increase of the particle's volume with respect to that from the hard core. An increase in particle volume, however, leads to a larger overall $F^{2}\left(k, \theta_{k}\right)$, since $F\left(k, \theta_{k}\right)$ scales to the particle volume. The net result may or may not be a decrease in amplitude of $S\left(k, \theta_{k}\right)$. Problems with the amplitude are even more apparent for films with a composition near a volume fraction of $1 / 2$. For example, at 18.2 at.\% Fe, figure 6.9 illustrates the $S\left(k, \theta_{k}\right)$ is approximately 2 times that observed experimentally.

The model spectra have also been computed for a 6.5 at.\% Mo sample, assuming two phases a-Ge and a-MoGe $e_{3}$. With ellipsoids of $(8,11)$ and $(10,12) \AA$ and $\eta=0.36$, the main features of the observed SAXS are reproduced (see figure 6.11). In this case, however, the computed scattering maximum is more than 2 times intense than that observed. The effects of polydispersity in the particle size and shape and in the features of the hard core are suspected to cause this effect. Even more striking, the magnitude of scattering predicted from this model of course peaks near 14 at.\% Mo (where the volume fraction of the two phases is $1 / 2$ ). However, the observed scattering does not appear to peak near 14 at.\% Mo. Figure 5.1 shows that the magnitude of scattering of the 6.5 and 12.4 at.\% Mo samples is approximately the same. At this point, it is not clear whether or not this inconsistency is due to the failure of the monodisperse ellipsoid model or if the structure of the material simply cannot be modeled by ellipsoids at volume fractions near $1 / 2$.

\subsection{Experimentally Obtained Interparticle Interference Function}

Although the Percus-Yevick interparticle interference function multiplied by the particle scattering reproduces the qualitative features of the data, we have also tried to 
extract the interparticle interference function directly from the data. The approach taken in this section is to determine the interparticle interference fur -tion by dividing out the particle scattering. Interparticle interference effects are not significant at large $k$, where the particle scattering dominates (see figure 6.7). Hence, by fitting the large $k$ data to scattering from particles, it might be possible to extrapolate the particle scattering to small $k$ and consequently divide it out of the recorded spectra. This is of course a difficult procedure: distributions in particle shape and size are unknown; only a limited region in $k$ has been recorded where the interparticle effects are small enough to ignore and the SNR is good enough to fit; and the particle scattering that is removed from the overall scattering to arrive at $S_{I N T}\left(k, \theta_{k}\right)$ will be, for a large part, extrapolated.

Since the scattering from monodisperse ellipsoids has large- $k$ ripples (e.g. see figure 6.4 and accompanying text), to fit the observed scattering (with no such ripples) to the particle scattering in the large $k$ region, we must consider the ellipsoids as polydisperse. Rigorously, the ellipsoids must be monodisperse in order to write the total scattering as a product of the particle form factor and interparticle interference functions (Eq. 6.3.3). A similar assumption is made for the atomic form factors--covalently-bonded $\mathrm{Si}$ or $\mathrm{Ge}$, for example, are not spherical atoms so there arises a problem of polydispersity. This is often ignored at least in the first approximation. The degree of polydispersity from the ellipsoids can be much greater, however, since the sizes can vary considerably while the basic dimensions of an atom are fairly well-defined.

To simplify the issue of polydispersity, we use Gaussians to define the degree of polydispersity:

$$
\left\langle F^{2}\left(\bar{k}, R_{1}, a_{1}, R_{3}, a_{3}\right)\right\rangle=A \int_{0}^{\infty} d r_{1} e^{-a_{1}^{2}\left(r_{1}-R_{1}\right)^{2}} \int_{0}^{\infty} d r_{3} e^{-a_{3}^{2}\left(r_{3}-R_{3}\right)^{2}} F^{2}\left(\bar{k}, r_{1}, r_{3}\right)
$$


with $F\left(\vec{k}, r_{1}, r_{3}\right)$ the form factor of an ellipsoid (Eq. 6.2.5) with axes $\left(r_{1}, r_{3}\right)$, normalization constant $A$, and polydispersity parameters $\left(a_{1}, a_{3}\right)$. The normalization constant can be readily determined:

$$
A=\frac{4}{\pi}\left[\begin{array}{c}
a_{1} a_{3} \\
{\left[1+\Pi\left(a_{1} R_{1}\right)\left[1+\Pi\left(a_{3} R_{3}\right)\right]\right.}
\end{array}\right.
$$

with $\Pi(u)$ the probability integral. Figure 6.12 illustrates the particle scattering for various degrees of polydispersity (i.e. several $a_{1}$ for $\theta_{k}=90^{\circ}$ ). The basic effect is a smoothing operation that allows us to smooth the large $k$ ripples.

The large $k$ limits of the scattering patterns for the 6.3 at.\% Fe sample have been compared to Eq. 6.4 .1 which contains 6 variables $\left(R_{1}, R_{2}, \rho_{p}\left(\rho_{1}-\rho_{2}\right)^{2}, a_{1}, a_{3}\right.$, constant background $B$ ). Although the experimental data have been placed on an absolute scale and background subtracted, any error in the subtraction or scale between subsequent oblique transmission measurements introduces an error that cannot be removed or fit. Figure 6.13 shows a fit for sample parameters along with the estimated interparticle interference functions. The values used in the fit are: $R_{1}=5.5 \AA, R_{2}=9.5 \AA, \rho_{p}\left(\rho_{1}-\rho_{2}\right)^{2}=1.310^{-6}$ $e^{2} / A^{9}, a_{1}=0.5, a_{3}=0.25$, and $B=-0.05$. With so many fitting parameters and a small $k$ range to be fit, it is difficult if not impossible to determine a unique solution with a nonlinear least squares fitting routine. The values chosen here are only used as a reasonable estimate for what we suspect the particle scattering might look like.

One immediate distinction between the Percus-Yevick interparticle interference function (figure 6.7) and that observed from the samples (figure 6.13c) is the amplitude of the first maximum as a function of $\theta_{k}$. The extracted interference function exhibits a strong maximum for $\theta_{k}=90^{\circ}$ which decreases dramatically and is quite small at $\theta_{k}=15^{\circ}$. The 

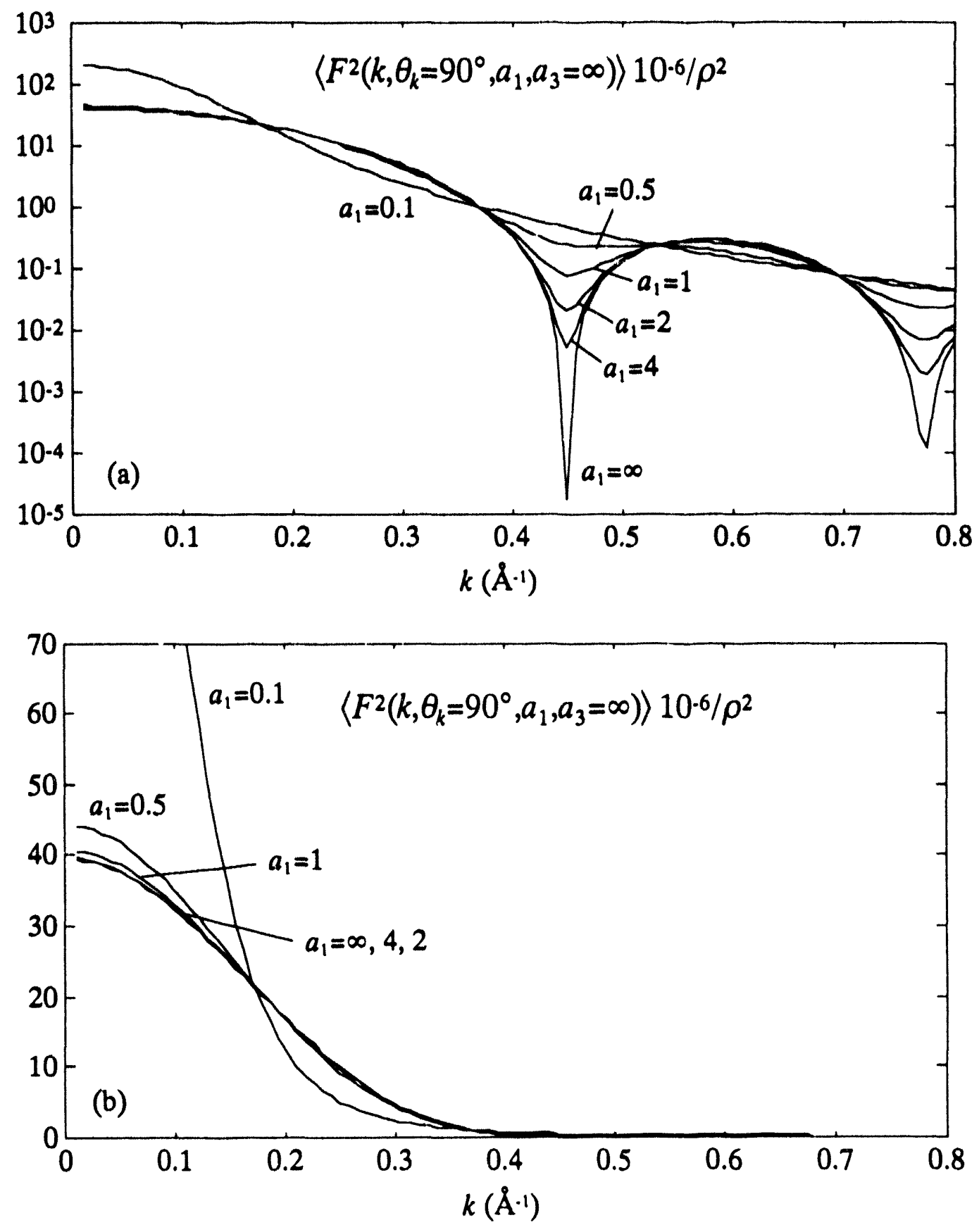

Figure 6.12. (a) Semi-log and (b) linear plots of scattering (for $\theta_{k}=90^{\circ}$ ) from Gaussian polydisperse ellipsoids of nominally $(10,15) \AA$. Influence of different polydisperse parameters $a_{1}$ is also shown. When $a_{1}$ and $a_{3}$ are both $\infty$, the ellipsoids are monodisperse. 

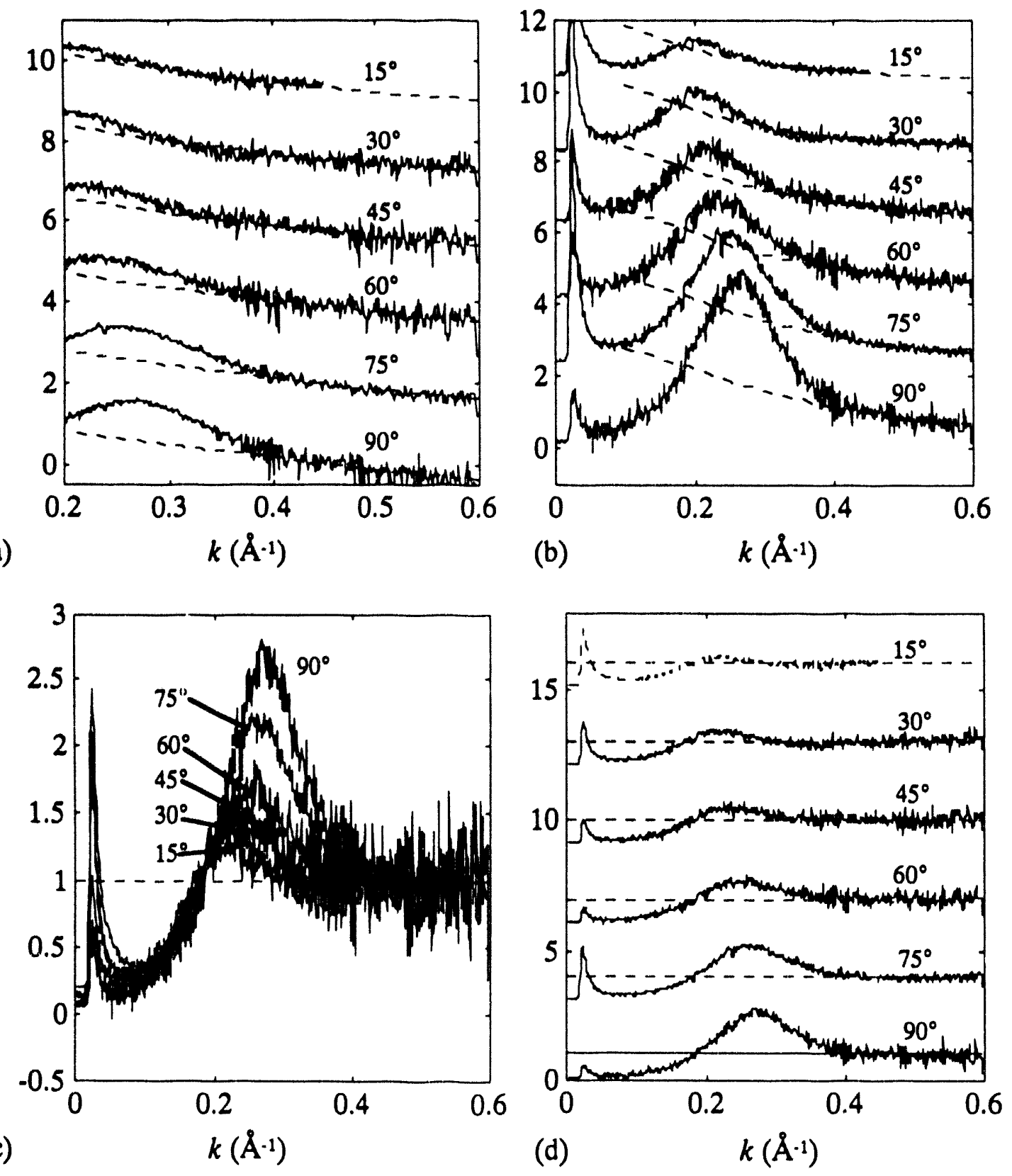

Figure 6.13. Semi-log (a) and linear (b) plots of best-fit particle scattering to the high-k scattering for the 6.3 at. \% Fe sample. Interparticle interference function $S_{I N T}\left(k, \theta_{k}\right)$, (c) and (d). Plots offset for clarity. 
amplitude of the first maximum in the Percus-Yevick model, on the other hand, is not $\theta_{k}$ dependent and is a result of the simple hard core potential that was used.

To investigate the origin of the interparticle interference function and obtain a realspace interpretation of the data, we have attempted to Fourier transform it and thus determine the particle's radial distribution function, $g\left(x, \theta_{x}\right)$. Due to the finite range in $k$ that has been recorded, the poor SNR, and the poor fits of the particle scattering at large $k$ (which also contribute to the large deviations around $S_{I N T}=1$ at large $k$ ), there has been little success in direct Fourier transformation of figure $6.13 \mathrm{c}$. Since the issue of polydispersity is also not well resolved and a qualitative understanding is that which is being sought, model spectra have been created that resemble the basic features of the interparticle interference function (figure 6.14a). By using a Gaussian-based function,

$$
S_{I N T}\left(k \cdot \theta_{k}\right)-1=\begin{array}{ll}
-1+A\left(\theta_{k}\right) e^{-300\left(k-k_{m}\left(\theta_{1}\right)\right)^{2}} & k \leq k_{m}\left(\theta_{k}\right) \\
\left(A\left(\theta_{k}\right)-1\right) e^{-300\left(k-k_{m}\left(\theta_{k}\right)\right)^{2}} & k \geq k_{m}\left(\theta_{k}\right)
\end{array}
$$

with $k_{m}\left(\theta_{k}\right)$ the position of the interparticle interference function maximum and $A\left(\theta_{k}\right)$ related to its amplitude, the basic features can be reproduced. This function is well-defined at small $k$ and for very large $k$, so the Fourier transform with the spherical harmonics approach will provide the radial distribution function without any large truncation errors (see figure 6.14a).

Figure 6.14b illustrates $\left(g\left(x, \theta_{x}\right)-1\right) \rho_{p}$ for the model spectra as a function of $\theta_{x}$. As mentioned previously in Section 6.3.a, $\rho_{p} g\left(x, \theta_{x}\right) d V$ is the number of particle centers that exist in the volume element $d V$ at $\vec{x}$ from a particle, averaged over all starting points of $\bar{x}$ (i.e. over all particles). For a model of disordered particles, $g(\bar{x})$ tends to 1 for $x$ on the order of a few particle diameters; that is, the number of particle centers from a given particle approaches the average value $\rho_{\rho} d V$ at large $\bar{x}$. In studies of isotropic amorphous materials, 

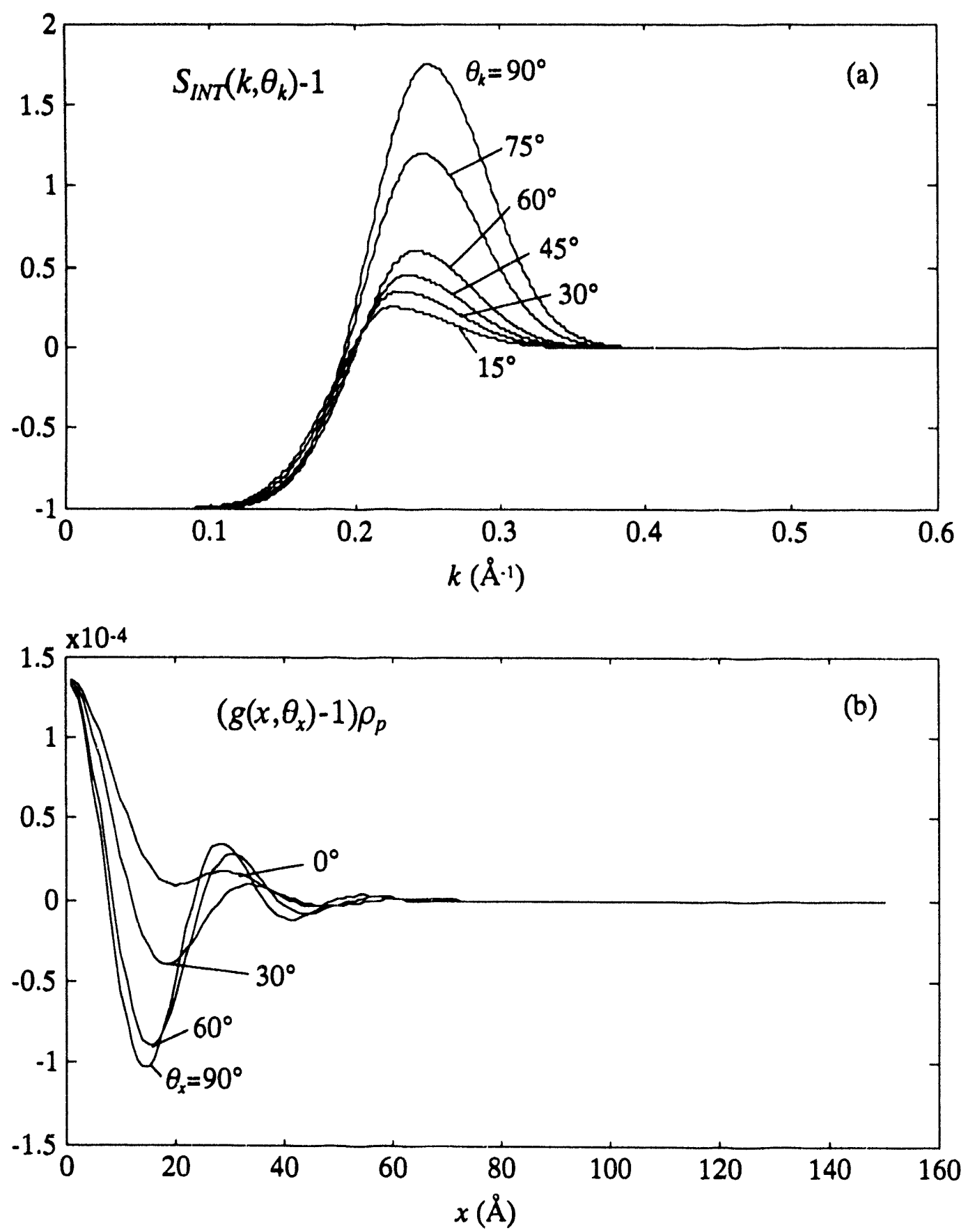

Figure 6.14. (a) Gaussian approximation to the experimentally observed interparticle interference function. (b) Fourier transform of (a) using the spherical harmonics approach outlined in Section 4.3. 

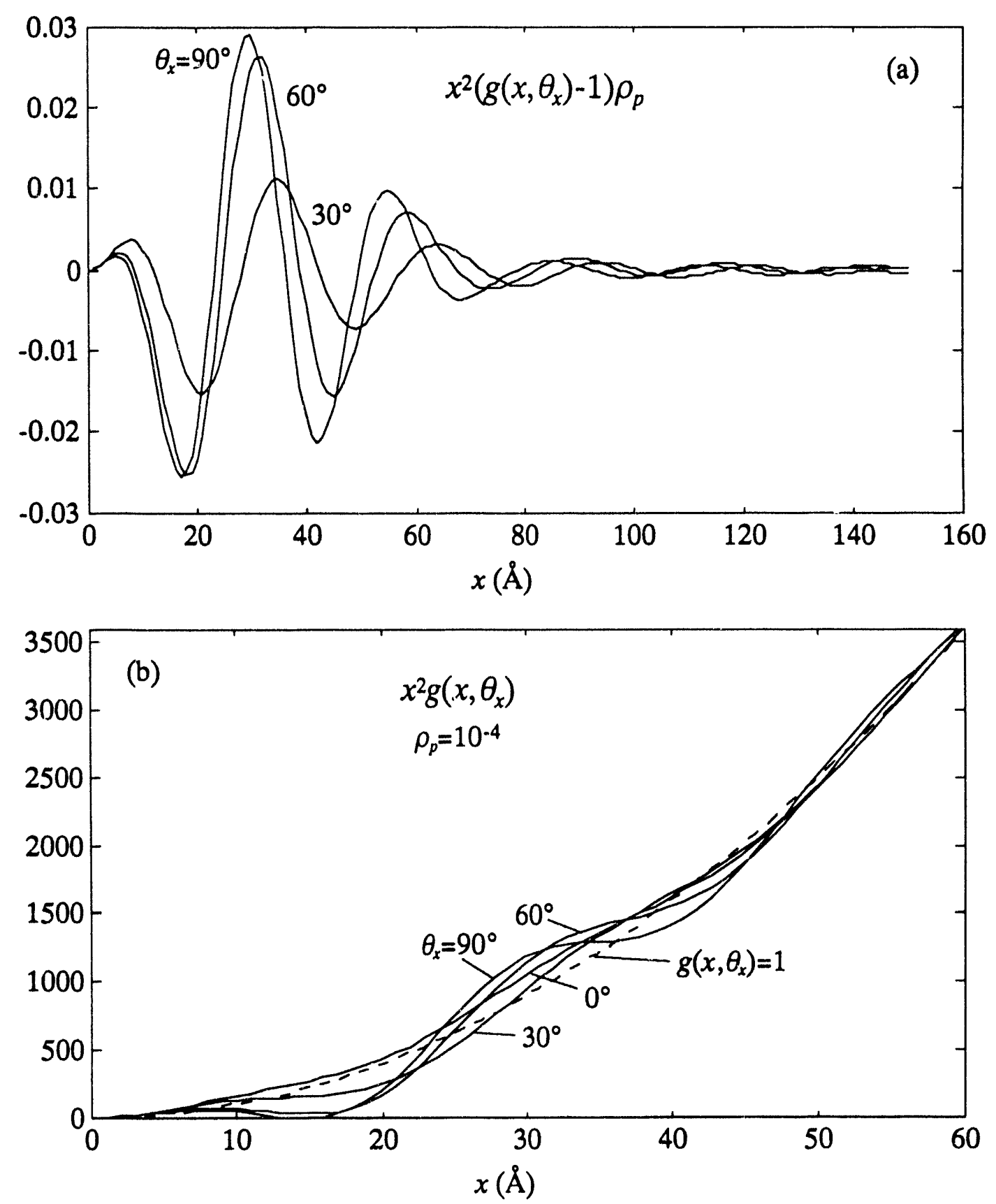

Figure 6.15. (a) Radial distribution function minus one, multiplied by $x^{2}$, along different film directions. (b) Radial distribution function, multiplied by $x^{2}$, along different film directions $\left(\rho_{p}-10^{-4}\right)$. 
the quantity $4 \pi x^{2} g(x)$ represents the number of particles (or, for example, atoms), on average, from another at a distance from $x$ to $x+d x$. With the cylindrically symmetric radial distribution function, the number of vectors that give an identical correlation between $x$ and $x+d x$ and $\theta_{x}$ and $\theta_{x}+d \theta_{x}$ is $2 \pi x^{2} \sin \theta_{x} g\left(x, \theta_{x}\right)$.

It is interesting to study $x^{2} g\left(x, \theta_{x}\right)$ since any oscillations in $g\left(x, \theta_{x}\right)$ are more readily observed with the greater weighting at large $x$, and without the $\sin \theta_{x}$ term, radial plots of $x^{2} g\left(x, \theta_{x}\right)$ should approach $x^{2}$ at large $x$ for a given $\theta_{x}$. Figure 6.15 shows the effect of multiplying the radial distribution function by $x^{2}$. In particular, the oscillations in realspace occur at greater length scales and wane in amplitude when the correlation direction moves from the film surface plane to the growth direction. With a particle density on the order of $10^{-4}$ (suggested by the inhomogeneous particle model and the size of the ellipsoids in the close-packed model), the function $x^{2} g\left(x, \theta_{x}\right)$ has been plotted along with the curve $x^{2}$ in figure $6.15 \mathrm{~b}$. The greatest ordering of particles is evident for a direction in the film surface plane. That is, the deep oscillations about the $x^{2}$ curve indicate that there is a highdegree of particle-particle short-range order which can be seen from the deviations at small $x$ from the large $x$ limit of $x^{2}$. As the particle correlation direction changes from 90 to 60 and then to $30^{\circ}$, the oscillations begin to die out and shift to larger distances. By $\theta_{x}=0^{\circ}$, it becomes difficult to distinguish oscillations of the radial distribution function around $x^{2}$, but they do appear to exist and at much smaller amplitude.

These results are consistent with the other models and the CCFs. The inhomogeneous and close-packed models along with the CCFs indicate that there exists an appreciable depletion region in the film surface plane surrounding regions of higher (lower) electron density than average. In the growth direction, however, such depletion regions are not as evident. The radial distribution function analysis of a model interparticle interference function that resembles what we suspect exists (given the limits of polydispersity, etc.) 
indicates that the particle correlations in the film plane are indeed much stronger and exhibit a short-range order which appears much weaker, if non-existent, in the growth direction. A comparison and summary of the different models and results will be presented in the next Chapter. 
References for Chapter 6

1D.J. Srolovitz and D.A. Kessler, "Phase Separation During Film Growth", unpublished (1989).

2J. Cahn, Acta Metall. 9, 795 (1961).

${ }^{3}$ A.L. Patterson, Phys. Rev. 56, 972 (1939).

${ }^{4}$ G.S. Cargill, Phys. Rev. Lett. 28, 1372 (1972).

${ }^{5}$ A. Guinier and G. Fournet, Small-Angle Scattering of X-Rays (W1ley: New York, 1955).

6J.S. Pederson, Phys. Rev. B 47, 657 (1993).

7L.S. Ornstein and F. Zernike, Proc. Akad. Sci. (Amsterdam) 17, 793 (1914).

${ }^{8}$ N.W. Ashcroft and J. Lekner, Phys. Rev. 145, 83 (1966).

${ }^{9}$ A.A. Broyles, S.U. Chung, and H.L. Sahlin, J. Chem. Phys. 37, 2462 (1962).

${ }^{10}$ G.S. Rushbrooke, J. Chem. Phys. 38, 1262 (1963).

11J.K. Percus and G.J. Yevick, Phys. Rev. 110, 1 (1958).

12M.S. Wertheim, Fhys. Rev. Iett. 10, 321 (1963).

${ }^{13 E}$ E. Thiele, J. Chem. Phys. 39, 474 (1963).

${ }^{14}$ R. Pynn, Solid State Comm. 14, 29 (1974).

15U.P. Singh and Y. Singh, Phys. Rev. A 33, 2725 (1986). J.F. Marko, Phys. Rev.

Lett. 60, 325 (1988).

16B.J. Berne and P. Pechukas, J. Chem. Phys. 56, 4213 (1972).

${ }^{17}$ A. Perera, P.G. Kusalik, and G.N. Patey, J. Chem. Phys. 87, 1295 (1987). 


\section{Chapter 7}

\section{Summary and Conclusions}

The primary goal of this work has been to address the issue of phase separation in amorphous Fe-Ge and Mo-Ge alloys. In particular, we have addressed the following questions:

1. What are the compositions of the phase-separating species?

2. What is the nature and source of the anisotropic scattering?

3. Are there any models that might explain such an anisotropy?

4. Is the phase separation influenced by the kinetics of the deposition process?

5. What is the relevance of the phase separation to the metal-insulator transition?

\subsection{Identification of Phase-Separating Species}

The anomalous scattering results for all oblique transmission angles were similar if not identical to the results of Rice et al. ${ }^{1}$ at normal incidence. That is, for amorphous $\mathrm{Fe}_{\mathrm{x}} \mathrm{Ge}_{1-\mathrm{x}}(\mathrm{x}<0.33)$, there is a subtle change in the scattering with changes in $\mathrm{x}$-ray energy near the Ge K-edge, but there is a large change in SAXS for energies close to the Fe Kedge. For the Mo-containing alloys, there is no noticeable change at the Ge $\mathrm{K}$-edge for all alloys studied, but we have observed the anomalous effect at the Mo K-edge for a 6.5 at.\% 
Mo sample (only one sample studied at this absorption edge). It should be mentioned, however, that a slight change was observed at the Ge K-edge through the weighted-average PSFs (figures 4.3 and 5.4), but the signal-to-noise ratio was not good enough to resolve the PSFs to determine any specific $\mathrm{Ge}-\mathrm{Ge}, \mathrm{Fe}-\mathrm{Ge}$, or Mo-Ge correlations.

The interpretation originally presented by Rice et al. ${ }^{1}$ for the anomalous scattering results was that the $\mathrm{Ge}$ atoms are homogeneously distributed with the metal atoms inhomogeneously distributed. When compared to the crystalline phases present in the Mo$\mathrm{Ge}$ and $\mathrm{Fe}-\mathrm{Ge}$ phase diagrams, the number density of $\mathrm{c}-\mathrm{Ge}$ number is within $5 \%$ of the number density of $\mathrm{Ge}$ in either $\mathrm{FeGe}_{2}$ or $\mathrm{MoGe}_{2}$. It was thus postulated that the anomalous scattering results are consistent with phase separation into regions of a-Ge and a-MGe $(\mathrm{M}=\mathrm{Mo}, \mathrm{Fe})$.

To test these ideas, we calculated the mean-square fluctuation of effective electron density for several samples. This is given by the pair correlation function at $x=0$ and is also known as the integrated intensity. Assuming two and three-phase models with amorphous $\mathrm{Ge}$ as one of the phases, we developed a means to determine the concentration of $\mathrm{Fe}$ in the second phase. Results from both the sharp and diffuse boundary models predict phase separation endpoints of a-Ge and a-FeGe ${ }_{2}$. The most Fe-rich sample which we have grown that shows SAXS is a 27 at.\% Fe sample (figure 4.2); the next samples we have are 44 and 47 at.\% Fe which show no appreciable SAXS over the studied $k$-range.

For the Mo-containing alloys, however, there is not enough data at the Mo K-edge to perform the same anomalous scattering analysis that we developed for the $\mathrm{Fe}-\mathrm{Ge}$ alloys. The conventional SAXS method still applies, and we determined (with the same assumptions as for the Fe-Ge alloys) that the endpoint compositions for the Mo-Ge alloys are a-Ge and an intermetallic close in stoichiometry to an a-MoGe ${ }_{3}$. Although an intermetallic $\mathrm{MoGe}_{3}$ does not exist in the equilibrium phase diagram, this result is consistent with the absence of a strong SAXS maximum in the 23.6 at.\% Mo sample and 
the work of Kortright and Bienenstock ${ }^{2}$ who argue that structural regions I and II meet at a composition close to 23-25 at.\% Mo.

Future experiments should focus on studying more samples at the Mo K-edge. With the limited beamtime and difficulty of ASAXS at the hard $\mathrm{x}$-ray energies, it was not possible to perform anomalous scattering measurements as a function of transmission angle $\theta_{k}$. Such measurements would help pinpoint the uncertainty of the endpoint composition. In addition, anomalous scattering measurements on the 23.6 at.\% Mo sample would help isolate the SAXS that is observed in figure 5.1. As mentioned previously in Chapter 5, there appears to exist a small maximum in the 23.6 at.\% Mo sample which is not well understood (background or chemical inhomogeneity). At this composition, one can suppose that there is still phase separation and that the a-Ge particles are fairly large. Then, one would have appreciable separation of the particles since the a-Ge component would be a small fraction of the entire sample. As a result, there would be little interparticle interference and one would expect scattering of that observed. These ideas should be tested in the future.

\subsection{Source of Anisotropic Scattering}

One of the most striking features of our analysis is that we seem to end up with virtually the same physical model no matter how we analyze the data. We have different ways of explaining the anisotropy, but they all seem to yield the same results. The most quantitative method is with the cylindrical correlation function (CCF), which has led to inhomogeneous and close-packed homogeneous particle models.

The Fourier transform of the anisotropic scattering provides information about the cylindrical correlation function, discussed in Sections 4.3 and 5.1, which clearly show that there are interparticle correlations in the film plane but not in the growth direction. The $k$ - 
space anisotropy is a result of regions of a particular electron density, set within a different electron density, that extend further in the growth direction than in the film surface plane ("in-plane" direction). From the results of the previous section, the different electron densities can be considered as that of a-Ge and either a-FeGe $\mathrm{F}_{2}$ or a-MoGe $\mathrm{M}_{3}$, with the elongated "particle" either a-Ge or the Ge-rich intermetallic (whichever occupies less than $1 / 2$ the volume fraction). The particle extends further in the growth direction than in-plane, by a factor of approximately $1.5: 1$ to $2: 1$, with basic particle axes for a 6 at.\% metal alloy approximately $12 \AA$ in the growth direction by $7 \AA$ in-plane for the Fe-Ge system and $9 \AA$ growth by $6 \AA$ in-plane for the Mo-Ge system.

As evidenced from the $\mathrm{CCF}$ for the 6.3 at.\% Fe sample, the particles are not correlated in the direction of growth. For an in-plane direction, the large negative regions in the CCF indicate that there is a "depletion region"; there exists great likelihood that the difference of electron density from the average is of opposite sign from particle interior to its immediate exterior. At even greater distances, the CCF indicates that particle-particle correlations also occur but with even smaller amplitude. In the growth direction, a depletion region does not appear to exist--the particle exterior is just the average clectron density. The CCF from a 6.5 at.\% Mo sample is similar, but a depletion region is also present in the growth direction as well as for an in-plane direction. In addition, the in-plane particle-particle correlation does not appear as strong as that for the amorphous $\mathrm{Fe}-\mathrm{Ge}$ samples.

The physical picture that thus emerges is one where the "particles" are formed within the layers by depleting the surrounding region of, for example, $\mathrm{Fe}$ or Mo atoms. Adatoms have a few seconds to rearrange on the surface before being covered by the advanced surface and governed by the much slower bulk transport coefficients. Then, fluctuations lead to little correlation between where the particles are formed in one layer and where they are formed after about $20 \AA$ of material have been deposited. This is consistent 
with figure 4.9a. There is no negative region at $\theta_{k}=0^{\circ}$, whereas there is an appreciable negative region at $90^{\circ}$. This analysis helps establish that the emerging surface plays a pivotal role in the degree and extent of the phase separation. In fact, by changing the deposition conditions in a simple way, we have grown amorphous $\mathrm{Fe}$-Ge films of identical composition that show different SAXS spectra. The different range of real-space correlations manifests itself in the changes in the position, breadth, and amplitude of the SAXS maximum. With mobilities greater and activation energies lower at the surface than in the bulk, it seems likely that most of the structural properties are determined at the surface, with only slight modification in the bulk (e.g. structural relaxáiion).

The inhomogeneous and close-packed particle models that we have constructed lead to the same conclusions. Since there is no appreciable positive bump in figures 4.9 and 5.3, interparticle interference effects may not be that important. The excluded volume that leads to a negative region in the CCF appears to be the most important part of the interparticle interference to model. Thus, the inhomogeneous ellipsoid model, with a depletion surrounding each inner region particle, automatically includes this most important effect and consequently can successfully reproduce the main features of the anisotropy. Interparticle effects between the composite particles is not important because the interparticle interference function deviates from one only at small angles. Since the scattering factor of each composite particle is going to zero at these small angles, the net contribution to the entire scattering from the interparticle function will thus be negligible.

The close-packed particle model explicitly includes the interparticle correlations, and the Percus-Yevick structure factor derived for ellipsoids also provides a qualitative agreement with the observed anisotropy. However, the Percus-Yevick model is a poor choice for the correlations that we observe. The interparticle correlations are not as well defined as that given from an equilibrium liquid structure factor from the Percus-Yevick 
theory, and the model does not incorporate any polydispersity of particle shapes and sizes that should greatly influence the breadth of the SAXS peak.

\subsection{Metal-Insulator Transition}

The inability to unambiguously define the critical composition at which the metalinsulator transition occurs is likely due to the anisotropic, nanoscale phase separation reported in this work. It appears likely that the different methods of sample preparation lead to various microstructures that change the electronic density of states and, hence, whether the alloy is considered metallic or insulating. Whether the transition is explained through a percolation or tunneling argument, the size and average distances between the metallic regions will define the sample's macroscopic electrical properties. Future experiments should focus on the kinetics of the deposition process in order to systematically vary growth parameters while studying the structure and electrical properties of the alloys. By coupling specific heat measurements with ASAXS, it might be possible to correlate the size and density of the metallic particles with the electronic density of states, observed by the linear term of the specific heat.

\subsection{New Questions}

In addition to the questions raised in the above sections, let us conclude with a discussion of a few last questions. The film is grown by passing a substrate table under a $\mathrm{Ge}$ sputtering gun and then under either a Mo or $\mathrm{Fe}$ gun. Our results indicate that fluctuations in the growth direction play a pivotal role in nucleating new, nanoscale particles with little correlation between where the particles are formed in one layer to where they are formed after $20 \AA$ of material has been deposited. Although for each pass under the targets only 0.2 to $0.8 \AA$ of material is deposited, one cannot help but wonder, however, if our results are related to this deposition technique. Would the same result be 
obtained if one had two targets pointed at the sample all the time, rather than rotating the sample in front of one target and then the other? How are the fluctuations that lead to nucleation at new sites related to the growth parameters and/or deposition geometry?

Finally, we have not addressed the question of phase separation in the $\mathrm{Fe}-\mathrm{Ge}$ samples with compositions greater than 33 at.\% $\mathrm{Fe}$. We do not find any indication of phase separation for these samples. Lorent $z^{3}$ and Rice ${ }^{4}$ have observed and studied phase separation in this system and composition range for samples in which the $\mathrm{Fe}$ was triodesputtered. For those samples with $x<0.33$, the triode-sputtered and magnetron-sputtered samples give rise to similar SAXS spectra. This raises the question as to whether those samples show evidence of the nonmagnetic to magnetic transition (at $\sim 40$ at.\% $\mathrm{Fe}$ ) in the same way that the ones studied in this thesis do. EXAFS data which were collected during the course of this work and Mossbauer measurements currently under way in Argentina ${ }^{5}$ may help resolve this question. 


\section{References for Chapter 7}

${ }^{1}$ M. Rice, S. Wakatsuki, and A. Bienenstock, J. Appl. Cryst. 24, 598 (1991).

2J.B. Kortright and A. Bienenstock, Phys. Rev. B 37, 2979 (1988).

3R.D. Lorentz, Ph.D. Thesis, Stanford University (1986).

${ }^{4} \mathrm{M}$. Rice, Ph.D. Thesis, Stanford University (in preparation).

${ }^{5}$ M.B. Fernandez van Raap, M.J. Regan, and A. Bienenstock, in preparation (1994). 

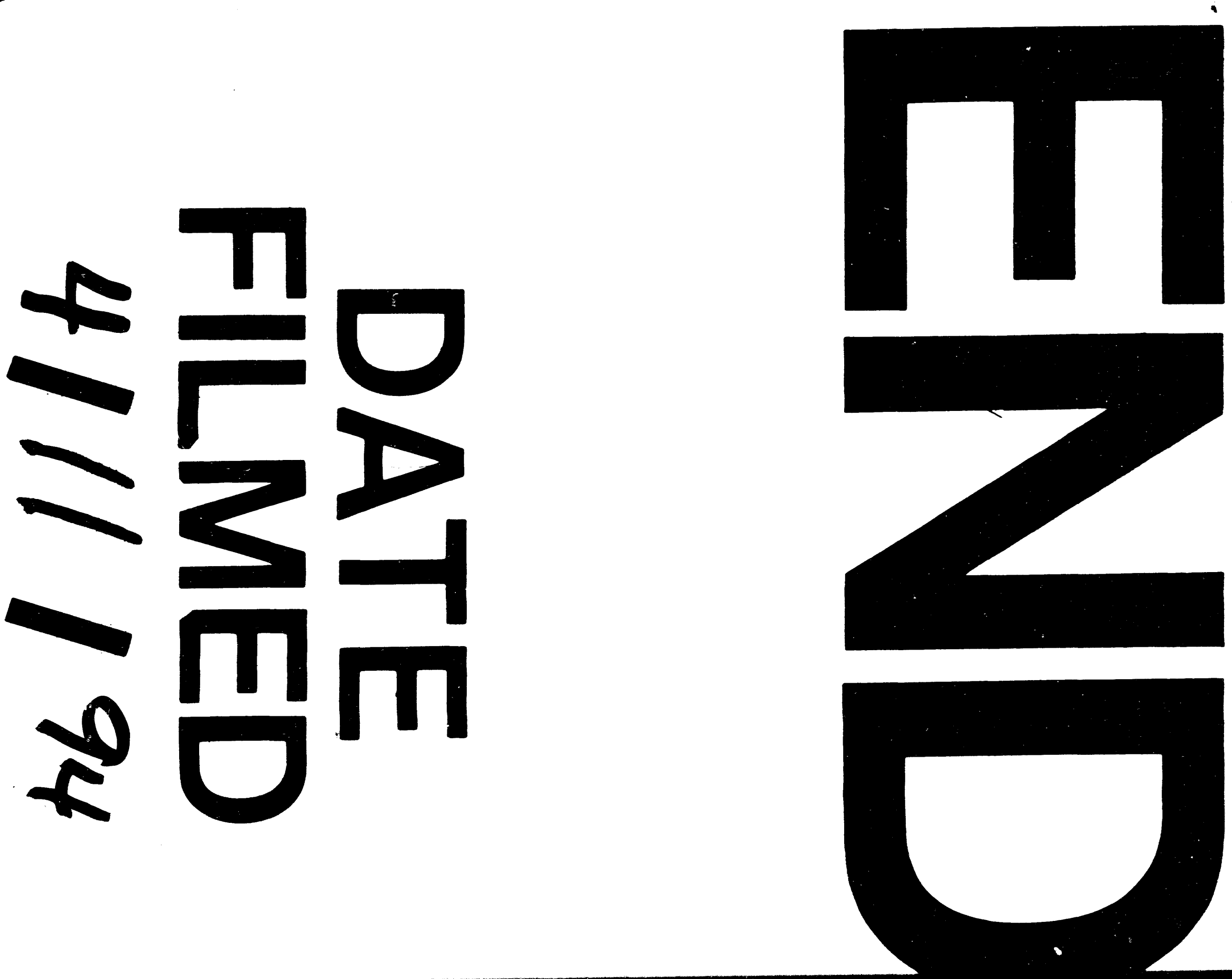
